Portland State University

PDXScholar

Spring 5-23-2016

\title{
Investigating the Effects of $17 \alpha$-Ethynylestradiol on Mitochondrial Genome Stability
}

\author{
Alicia M. Chivers \\ Portland State University
}

Follow this and additional works at: https://pdxscholar.library.pdx.edu/open_access_etds

Part of the Biology Commons, Genetics and Genomics Commons, and the Toxicology Commons Let us know how access to this document benefits you.

\section{Recommended Citation}

Chivers, Alicia M., "Investigating the Effects of $17 \alpha$-Ethynylestradiol on Mitochondrial Genome Stability" (2016). Dissertations and Theses. Paper 2989.

https://doi.org/10.15760/etd.2990

This Thesis is brought to you for free and open access. It has been accepted for inclusion in Dissertations and Theses by an authorized administrator of PDXScholar. Please contact us if we can make this document more accessible: pdxscholar@pdx.edu. 
Investigating the Effects of $17 \alpha$-Ethynylestradiol on

Mitochondrial Genome Stability

by

Alicia M. Chivers

A thesis submitted in partial fulfillment of the requirements for the degree of

\author{
Master of Science \\ in \\ Biology
}

Thesis Committee:

Kim H. Brown, Chair

Jeffrey M. Duerr

Jason E. Podrabsky

Portland State University

2016 


\begin{abstract}
Environmental toxicants are ubiquitous throughout the environment as a result of human activity. Among these toxicants, environmental estrogens are a category of particular concern due to their environmental prevalence and potency in altering reproductive traits. While many studies have addressed the detrimental effects of environmental estrogens on both aquatic and terrestrial organisms, few have analyzed the potential for these compounds to alter mitochondrial function. Mitochondria are the primary energy-generating system for all eukaryotic life, supporting all aspects of development, metabolism, and growth. Each cell within the body contains many mitochondria which in turn contain multiple copies of their own DNA genome, mitochondrial DNA (mtDNA). Mutations in mtDNA are responsible for a wide range of human diseases such as metabolic syndromes, cancers, and obesity. Among these mitochondrial diseases many are characterized by increased levels of heteroplasmy, multiple mitochondrial DNA haplotypes within an individual. Increased heteroplasmy can alter normal mitochondrial function and influence disease initiation and progression.
\end{abstract}

To date, no studies have investigated the effects of synthetic estrogens on mitochondrial genome stability. Synthetic estrogens have the capacity to bind to estrogen receptors and initiate estrogenic responses through translocation into the mitochondrion. Despite our knowledge about the relationship of heteroplasmy and disease, we still do not have a complete grasp of the mechanisms of heteroplasmic induction. Here we report our analysis of the effects of $17 \alpha$-ethynylestraiol (EE2) exposure in three studies to investigate its effect on mitochondrial genome stability. Data analysis reveals a 
statistically significant relationship between EE2 exposure and increased heteroplasmic frequency. 


\section{For Sandra Lou Chivers}

I dedicate this work to my late mother, Sandra L. Chivers, whose thirst for knowledge and quest for discovery continues to inspire me daily. 


\section{Acknowledgements}

I'd like to thank the following for their part in supporting me through this work, Advisor, Dr. Kim H. Brown

Committee members, Dr. Jason E. Podrabsky and Dr. Jeff M. Duerr

Funding sources, Forbes Lea Endowment and in part by the National Institute of Health K99/R00 ES018892

Lab-mates and fellow graduate students: Josh Faber-Hammond, Lindsay Holden, Josiah Wagner, Amie Romney, Claire Riggs, Ian Garrett, Stephen Christy, and Alisha Beirl Undergraduate help: Aleks Weir, Emma Houssein, Zachary Neumann, and Juan Sanchez Jimenez

Collaborative help: Dr. Andrea DeBarber and Dr. Irvine Schultz Friends and family

My dearest Douglas Brittain 


\section{Table of Contents}

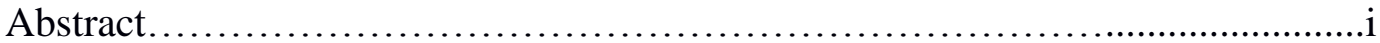

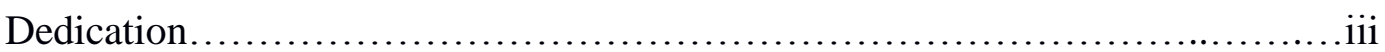

Acknowledgements........................................................

List of Tables...........................................................

List of Figures.................................................... vii

Chapter I: Overview and Background 1

Chapter II: Preliminary study on trout

$\begin{array}{ll}\text { Introduction } & 13\end{array}$

$\begin{array}{ll}\text { Methods } & 15\end{array}$

$\begin{array}{ll}\text { Results and Conclusions } & 17\end{array}$

$\begin{array}{ll}\text { Figures and tables } & 19\end{array}$

Chapter III: Acute treatment study and baseline comparison

$\begin{array}{ll}\text { Introduction } & 22\end{array}$

$\begin{array}{ll}\text { Methods } & 24\end{array}$

$\begin{array}{ll}\text { Results and Conclusions } & 31\end{array}$

$\begin{array}{ll}\text { Figures and tables } & 41\end{array}$

Chapter IV: Chronic treatment study

Introduction $\quad 65$

$\begin{array}{ll}\text { Methods } & 66\end{array}$

$\begin{array}{ll}\text { Results and Conclusions } & 70\end{array}$

$\begin{array}{ll}\text { Figures and tables } & 75\end{array}$

Chapter V: Discussion $\quad 83$

$\begin{array}{ll}\text { References } & 85\end{array}$ 


\section{List of Tables}

Table 1: Number of heteroplasmic sites per individual 20

Table 2: Acute exposure (2) sequencing sample key 42

Table 3: LC-MS between and within run comparison of calibrants QCs 47

Table 4: LC-MS calculated mean EE2 concentrations $\quad 48$

Table 5: Average sequence coverage for acute exposure (2) maternal fish $\quad 49$

Table 6: Average sequence coverage for acute exposure (2) AB pairs 49

Table 7: Average sequence coverage for acute exposure (2) Tu pair 49

Table 8: Average sequence coverage for acute exposure (2) WIK pairs $\quad 50$

Table 9: Average sequence coverage for acute exposure (2) baseline fish $\quad 50$

Table 10: Alignment scores for acute exposure (2) AB pairs 52

Table 11: Alignment scores for acute exposure (2) Tu pair 53

Table 12: Alignment scores for acute exposure (2) WIK pairs $\quad 54$

Table 13: Alignment scores for acute exposure (2) baseline fish 55

Table 14: Alignment scores for acute exposure (2) maternal fish 56

Table 15: Comparison of acute exposure (2) replicate error rate 58

Table 16: Top ten sites with greatest heteroplasmic variance bt reps 58

Table 17: Phenotypic comparison of control vs treated fish at 8 weeks 82 


\section{List of Figures}

Figure 1: Schematic of cellular respiration 3

Figure 2: Schematic of electron transport chain $\quad 4$

Figure 3: PCR amplification for genomic DNA contamination 19

Figure 4: Linear regression of heteroplasmy between sequence replicates $\quad 19$

Figure 5: Heteroplasmic sites between control and replicates 20

Figure 6: Map of heteroplasmic sites on UCSC genome browser 21

Figure 7: Diagram of acute exposure system $\quad 41$

Figure 8: Acute exposure design $\quad 41$

Figure 9: Survival curves for acute exposure series (1) and (2) control fish $\quad 42$

Figure 10: Survival curves for acute exposure series (1) and (2) 10ng EE2/L $\quad 44$

Figure 11: Survival curves for acute exposure series (1) and (2) 100ng EE2/L $\quad 45$

Figure 12: Survival curves for acute exposure series (1) and (2) 250ng EE2/L 46

Figure 13: Proportion of females between control and 10ng EE2/L treated fish 47

Figure 14: Examples of gel verification for DNA fragment target size 48

Figure 15: Example of quality control check for DNA fragment target size $\quad 48$

Figure 16: Overlapped sequence coverage between three replicate samples $\quad 51$

Figure 17: Linear regression of heteroplasmy between sequencing replicates $\quad 57$

Figure 18: Breakdown of heteroplasmic sites per individual in baseline fish $\quad 59$

Figure 19: Breakdown of heteroplasmic sites in baseline fish 59

Figure 20: Breakdown of heteroplasmic sites: AB maternal, control and treated 60

Figure 21: Breakdown of heteroplasmic sites: Tu maternal, control and treated 60 
Figure 22: Breakdown of heteroplasmic sites: WIK maternal, control \& treated 61

Figure 23: Breakdown of heteroplasmic sites: maternal lines from each strain $\quad 61$

Figure 24: Heteroplasmic sites by pair and strain in treated vs control 62

Figure 25: Heteroplasmic sites in treated vs control 63

Figure 26: Heteroplasmic sites across all groups $\quad 64$

Figure 27: Survival curves for chronic exposure, control 75

Figure 28: Survival curves for chronic exposure, 1ng EE2/L 76

Figure 29: Survival curves for chronic exposure, 10ng EE2/L 77

Figure 30: Survival curves for chronic exposure, 25ng EE2/L 78

Figure 31: Survival curves for chronic exposure, 50ng EE2/L 79

Figure 32: Proportion of females between control and chronic treated fish $\quad 79$

Figure 33: Gross image of control larva at 8 weeks $\quad 80$

Figure 34: Gross image of 1ng EE2/L treated larva at 8 weeks 80

Figure 35: Gross image of 10ng EE2/L treated larva at 8 weeks 80

Figure 36: Gross image of 25ng EE2/L treated larva at 8 weeks 81

Figure 37: Gross images of 50ng EE2/L treated larvae at 8 weeks 81

Figure 38: Histological image of intersex gonadal tissue 82 


\section{Chapter I: Overview and Background}

Mitochondria are vital intracellular organelles, central to most eukaryotic life. They are best identified for their role in the production of cellular energy. This efficient system coordinates energy production based on the availability of organic molecules and oxygen to meet energy demands. They are also key to a continuum of other essential cellular functions. Multiple cell wide stress responses are coordinated by mitochondria and they play a principal role in both cellular life and cellular death. They support all aspects of development, metabolism, and growth and are vital in the maintenance of adult tissue homeostasis (Nunnari \& Suomalainen, 2012; Youle et al., 2012).

Mitochondrial origins represent one of the earliest known symbioses occurring $>1.5$ billion years ago between a proto-mitochondrion and a host prokaryote cell(Brown, 2008; Gray, 1999). This event marked the initiation and diversification of eukaryotic life itself. Over time, this symbiosis evolved into an irreversible merger of mitochondria into the host cytoplasmic organelle system and eukaryotes are marked by the co-occurrence of two complete genomes, nuclear (e.g., host) and mitochondrial in animals. Genes with duplicate function between symbiotic partners became redundant and as part of the symbiotic inclusion process, the mitochondrial genome underwent a genome reduction in animals either through gene transfer and/or loss of duplicate genes. This reduction in size has resulted in extremely compact mitochondrial genomes, particularly in vertebrates, with overlapping coding sequences essential to organelle function (Brown, 2008; Gray, Burger, \& Lang, 1999; Timmis, Ayliffe, Huang, \& Martin, 2004). 
Structurally mitochondria have two membranes, an outer and inner, required for normal function. The outer membrane encloses the entire organelle and contains a large number of membrane proteins which allow smaller molecules and ions to pass freely to the inner side of the outer membrane. The inner mitochondrial membrane is a highly convoluted structure with folded invaginations, cristae, which increases the inner membrane surface area. This membrane is highly impermeable and contains the gelatinous matrix rich in ions, proteins, and enzymes involved in cellular metabolic processes (e.g., tricarboxylic acid cycle). Also, embedded within the inner membrane are numerous protein complexes, sites of metabolic pathways (e.g., oxidative phosphorylation) happening entirely or in-part within the mitochondria (Gray, 1999; Schapira, 2006; Wallace \& Fan, 2011).

Both the inner mitochondrial membrane and matrix are central to mitochondrial metabolic processes. Of these, the most important is the synthesis of adenosine triphosphate (ATP) through oxidative phosphorylation (OXPHOS). OXPHOS, the final step in aerobic cellular respiration (Figure 1) generates a net 36 ATP at a cost of 1 glucose molecule. Simplified, this process begins with the import of the glycolytic product pyruvate into the mitochondrial matrix. Pyruvate is reduced to mitochondrial acetyl coenzyme-A (acetyl coA), $\mathrm{CO} 2$ and NADH. From there, acetyl-coA enters the tricarboxylic acid cycle (TCA cycle, i.e., citric acid or Kreb cycle) where it's converted to metabolic intermediates through a concentrated series of redox reactions, occurring within the mitochondrial matrix. During the TCA cycle, the reduction of organic acid intermediates produces excess NADH and FADH2 which are the predominant electron 
carriers used in the electron transport chain (Wallace, Fan, \& Procaccio, 2011; Wallace \& Fan, 2011).

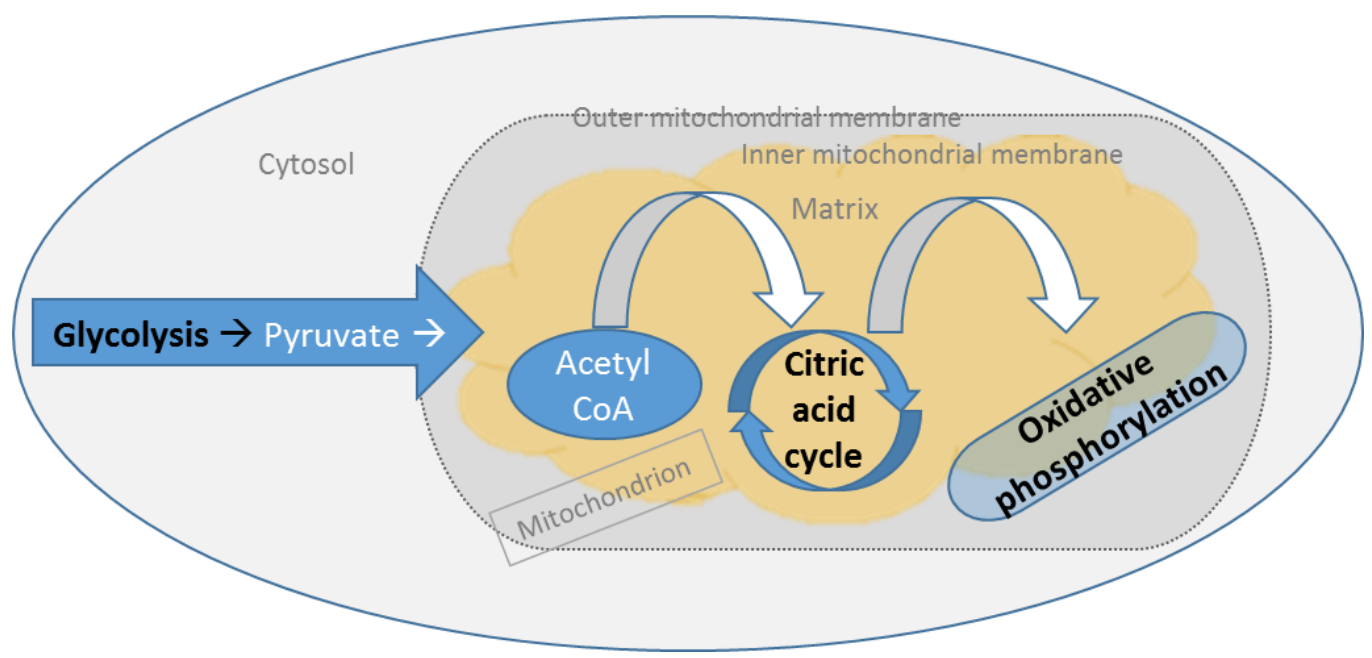

Figure 1: Schematic of cellular respiration. The glycolytic product pyruvate is imported into the mitochondrial matrix, reduced acetyl coenzyme-A where it enters the citric acid cycle (TCA). During the TCA cycle, acetyl CoA produces electron carriers used in the electron transport chain (within the inner mitochondrial membrane), the site of oxidative phosphorylation producing a net 36 molecules of cellular energy, ATP.

The electron transport chain is composed of a series of embedded inner mitochondrial membrane protein complexes that work to transfer electrons with the concomitant extrusion of proton ions. Working as electron carriers, NADH and FADH2 are oxidized as they supply electrons to $\mathrm{O} 2$. Protons are pumped out of the mitochondrial matrix into the intermembrane space as electrons are passed along the transport chain. This process creates an electrochemical gradient that results in energetically favorable phosphorylation of $\mathrm{ADP}$ and phosphate into ATP as protons flow back into the mitochondrial matrix (Figure 2). The highly impermeable inner mitochondrial membrane 
is key in creating an electrochemical gradient between the matrix and intermembrane space (Duchen, 2004; Nunnari \& Suomalainen, 2012).

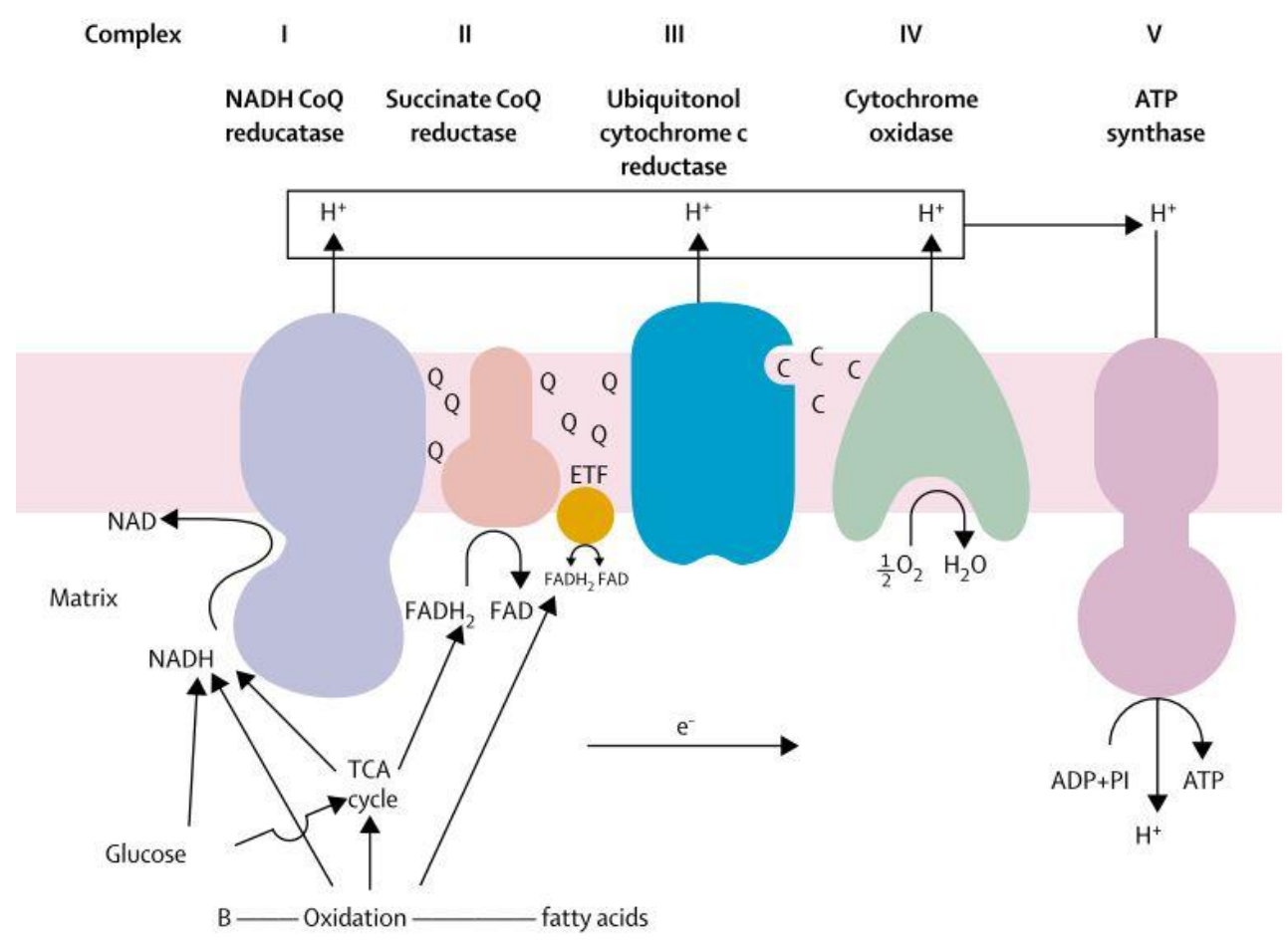

Figure 2: Schematic of electron transport chain showing the embedded inner mitochondrial membrane proteins transfer of electrons and concomitant extrusion of protons. This processes creates an electrochemical gradient that results in the phosphorylation of ADP and phosphate into ATP as protons flow back into the mitochondrial matrix (Schapira, 2006).

In addition, other catabolic pathways contribute to the production of ATP within the mitochondria through the catabolism of other macromolecules In the process of $\beta$ oxidation, fatty acids are degraded in the mitochondria to generate acetyl-CoA units which feed the TCA cycle. The mitochondrial enzyme pyruvate oxaloacetate is involved in the conversion of metabolic intermediates involved in gluconeogenesis. Even ketogenic processes occur entirely within the mitochondria during periods of high $\beta$ - 
oxidative activity and low TCA activity where acetyl-coA is converted to ketone bodies (Kroemer et al., 2012; Nunnari \& Suomalainen, 2012).

Reactive oxygen species (ROS) are a normal byproduct of metabolic pathways occurring within the mitochondria with the most prominent source being cellular respiration. Excess production can cause oxidative stress resulting in damaged mitochondrial proteins, lipids, and DNA. Damaged cellular components are causal to metabolic dysfunction, alterations in membrane potential, and further increase the production of ROS. These aberrant conditions can increase ATP consumption to compensate for membrane potential deviations, further perpetuating adverse cellular conditions. Repair and quality-control mechanisms safeguard mitochondria from the detrimental or deleterious effects of oxidative stress. In response to genotoxic damages caused by ROS, mitochondria initiate some forms of DNA repair pathways similar to those in the nucleus (Gladyshev, 2013).

Normal mitochondrial activity is dependent on the functioning of over a thousand proteins. These proteins are encoded mostly by genes within the nuclear genome (nDNA); however, each individual mitochondrion contains multiple (i.e., between 5-10) circular genomes necessary for its function. The mitochondrial genome (mtgenome or mtDNA) is highly compact with no introns and very few intergenic spaces. In vertebrates, it ranges from 15,000-17,000 kilobases $(\mathrm{kb})$ in length and encodes for 37 genes necessary respiratory chain activity and translation of mtDNA genes (Broughton, Milam, \& Roe, 2001; Kelly \& Scarpulla, 2004). 
Given the central role in mitochondria in most cellular processes, impaired mitochondrial function is causal to a variety of diseases. These phenotypes range from alterations in cell function to cell death as a result of mutations in mtDNA. Mitochondrial disease can be heterogeneous or multisystemic, but often preferentially affects tissues or systems with high-energy demands including the brain, heart, muscle, digestive and endocrine systems. Severity of disease states is often related to mtDNA copy number (i.e., the proportion of mutated to wild-type mtgenomes). The mixture of mutant and wildtype (i.e., normal) mtDNA genomes within a single mitochondrion, cell, tissue or individual is termed heteroplasmy (Chan, 2006; Jayaprakash et al., 2014; Nunnari \& Suomalainen, 2012; Wallace \& Chalkia, 2016).

Given mitochondria's strict pattern of maternal inheritance, heteroplasmy can occur from germ-line inheritance, (i.e., from oocyte cytoplasm), or arise as somatic mutations (Brown, 2008). If a heteroplasmic oocyte is fertilized and forms a zygote, during each cell division different mitochondrial genomes (i.e., heteroplasmy) can be randomly distributed to the daughter cells and continue to be copied during each cell cycle. Unequal distribution can contribute to mitochondrial disease initiation and onset. When mutant mtDNA is inherited through oocyte transmission, the proportion of mutated mtgenomes, as well as replication and distribution, is stochastic (Wallace \& Fan, 2011). Over many cell generations, heteroplasmy can increase (i.e., higher proportions of mutant mtDNA) and consequently those cells undergo a decrease in the production of energy (Wallace \& Chalkia, 2016). 
Somatic mutations can occur in mtDNA just as they do in nuclear DNA. Mitochondria have a high mutation rate as they lack protective histones like nuclear DNA and have only rudimentary repair mechanisms. Mitochondria undergo fission and fusion events as a mechanism of redistribution to meet altered energetic demands but also to selectively remove stressed or damaged organelles. MtDNA molecules exist in clusters called nucleoids adjacent to the respiratory chain where they are prone to oxidative nucleotide base damage from ROS byproduct production. Mutated mtgenomes have the ability to expand clonally, eventually causing an energetic defect (Chandra \& Singh, 2011; Schmitt et al., 2012).

Energetic defects in both inherited and somatic mutated mtgenomes have been implicated in forms of blindness, deafness, movement disorders, dementias, cardiomyopathy, myopathy, renal dysfunction and aging (Chan, 2006; Chandra \& Singh, 2011). There is an ever growing list of disease phenotypes being characterized by mitochondrial dysfunction with ill-understood patterns of disease initiation and progression (Payne et al., 2013). Currently there is no pharmaceutical cure for any mitochondria-related diseases (Nunnari \& Suomalainen, 2012). With the severity of most mitochondrial diseases states, this organelle is an emerging topic of study.

Although mitochondria have been studied as a therapeutic target (Wallace et al., 2011), few studies have assessed the effects of environmental toxicants on mitochondria. Environmental toxicants are ubiquitous in our environment with continuous release from various sources including industrial and consumer activity (Meyer et al., 2013). Among these, environmental estrogens are of particular concern given their prevalence in a wide 
range of products (i.e., pesticides, plastics, pharmaceuticals and household products) and potent estrogenic activity that modulates and/or disrupts a variety of animal systems (Hecker \& Hollert, 2011; Tyler, Jobling, \& Sumpter, 1998). Many studies have demonstrated environmental estrogens induce physiological and developmental dysfunction on both aquatic and terrestrial species at environmentally relevant concentrations.

Two of the best exemplified studies are a 7-year whole lake experiment in Ontario, Canada and the analyses of English river systems receiving effluent from wastewater facilities. In the whole lake exposure Kidd and colleagues demonstrated that chronic exposure of $17 \alpha$-ethynylestradiol (i.e., synthetic estrogen) at environmentally relevant concentrations led to the near collapse of the lake fathead minnow (Pimephales promelas) population. The decline was linked to both the feminization of males, which reduced levels of sperm production, and altered oogenesis in females, which resulted in decreased offspring survival (Kidd et al., 2007).

Other studies investigating the impacts of estrogenic contaminants on aquatic species have observed aneuploid sperm formation (Brown, Schultz, Cloud, \& Nagler, 2008) and reduced embryonic survival following parental exposure in rainbow trout (Brown, Schultz, \& Nagler, 2007), reduced female reproductive success in zebrafish (Coe, Söffker, Filby, Hodgson, \& Tyler, 2010) and intersex males (i.e., phenotypic male with gonads containing both testicular and ovarian tissue) in roach (Flores \& Hill, 2008; Tyler \& Jobling, 2008). Such effects are not limited to aquatic organisms with similar studies conducted in mice and rats having also shown developmental disturbances and 
reproductive anomalies including: reduced sperm counts in males (Takahashi \& Oishi, 2006), increased oocyte aneuploidy (Susiarjo, Hassold, Freeman, \& Hunt, 2007), reduced sex accessory organ weights (Takahashi \& Oishi, 2006), and disruptions in oogenesis (Hunt \& Hassold, 2008). Similar to the findings in aquatic vertebrates, many of the observed detrimental effects in terrestrial models also occurred with environmentally relevant doses.

Environmental estrogens, including synthetic estrogens and estrogen mimicking compounds, are of particular concern due to their environmental prevalence and potency in altering reproductive traits. Contaminants originate from a variety of sources with one of the most prominent mechanisms of environmental release occurring through wastewater effluent (Desbrow \& Routledge, 1998; Hamid \& Eskicioglu, 2012; Pollock, Dubé, \& Schryer, 2010; Ternes et al., 1999). Synthetic estrogens, such as $17 \alpha-$ ethynylestradiol (EE2), are widely used as pharmaceutical contraceptives for humans and animals as well as for hormone replacement therapy for post-menopausal women (Rogan \& Gladen, 1985).

Vertebrate animals, including humans, primarily excrete estrogens through the urinary system in a conjugated and biologically inactive form. This conjugated, inactive form is highly soluble in water and easily transported in waste water. Despite this, the active unconjugated form of estrogen is the dominant form found in the influent and effluent of waste water treatment facilities. The process of converting the compounds from inactive to active forms after excretion occurs through an enzymatic process with bacteria de-conjugating the estrogens and converting them to their biologically active 
forms. This fluctuation between the inactive and active form of estrogen compounds results in inaccurate detection and reduced efficacy of removal processes at waste water treatment plants. Although these treatment facilities still reduce the concentrations following waste water treatment, environmental estrogenic effects are still observed at low doses on a variety of species (Ingerslev, Vaclavik, \& Halling-Sørensen, 2003; Kolpin et al., 2002; Schultz, Orner, Merdink, \& Skillman, 2001).

Although there are clear indications that environmental estrogens have direct effects on development and reproductive functions, few studies have analyzed the potential for these compounds to alter mitochondrial genomic structure despite mitochondria having the ability to bind estrogen. Endogenous estrogens bind to estrogen receptors (ERs) eliciting complex cellular interactions through membrane, nuclear, and mitochondrial signaling pathways (Ropero, Alonso-Magdalena, Ripoll, Fuentes, \& Nadal, 2006). The import of ERs into the mitochondria (mtERs) allows them to modulate estrogenic activity within mitochondria, coordinate mitochondria-nuclear regulatory cross-talk, and affect overall concentration and distribution of anti-apoptotic proteins (Kaipparettu et al., 2013; Spinazzola \& Zeviani, 2007). An environmental estrogens capacity to bind ERs and elicit estrogenic responses is subject to the chemical structure of the foreign estrogen and ER binding affinities within target tissues (Shyu, Cavileer, Nagler, \& Ytreberg, 2011). 17- $\alpha$ ethynylestradiol (EE2) is a potent estrogenic compound in many fish species with greater binding affinity compared to the endogenous estrogen estradiol-17 $($ E2). 
Danio rerio, zebrafish, are a small freshwater tropical fish originating from East India. Zebrafish have become a valuable vertebrate model organism used in various areas of study; developmental, reproductive, genetic, toxicological, and biomedical (Brown et al., 2012; Soares et al., 2009). There are multiple advantages to using zebrafish as a study model compared to other common vertebrate models (e.g., mice). Among these, the most obvious are that zebrafish are relatively inexpensive to maintain, produce a large number of offspring per mating and have a rapid generation/gestation period. Zebrafish embryos develop outside of the mother and development processes are fairly visible due to the transparency of the egg membrane.

In zebrafish, both the nuclear and mitochondrial genomes have been fully sequenced (Broughton, Milam, \& Roe, 2001). The use of zebrafish in studies investigating the mitochondrial genome is particularly useful because of its many commonalities to other vertebrates. Zebrafish mtgenome gene order and content are identical to the majority of vertebrates, including humans, making them a valuable tool for studying mitochondrial disease states (Howe et al., 2013).

Lastly, several laboratory strains of zebrafish exist which are useful in understanding heterogeneity (Gladyshev, 2013). Of these strains, the three most common laboratory strains include $\mathrm{AB}, \mathrm{Tu}$, and WIK. Each strain differs in their initial development and historic degree of selective breeding. Continued examination of mtDNA variation between strains is necessary for future work using zebrafish as a model given mtDNA strain variation may have a role in interstrain phenotypic variation. 
To date, no studies have assessed the potential for induced heteroplasmy as a result of environmental estrogen exposure. Given that environmental estrogens have the potential to cause incidental mitochondrial genomic damage following exposure via binding to estrogen receptors and translocation into the mitochondrion (Menuet et al., 2004), we investigated the effects of 17- $\alpha$ ethynylestradiol (EE2) exposure on mitochondrial genome stability. The following studies investigated whether mitochondrial heteroplasmy can result from non-native estrogen exposure in fish through the following aims: (1) preliminary assessment of short-term EE2 exposure on aged isogenic male trout mitochondrial DNA, (2) acute EE2 exposure effects on mitochondrial DNA during sexual differentiation on full-sib offspring using three strains of zebrafish with comparisons to baselines rates from aged strain specific fish and (3) assess the effects of chronic EE2 exposure on mitochondrial DNA during full-sib offspring development in three strains of zebrafish. 


\section{Chapter II: Preliminary study on trout}

\section{Introduction}

Environmental estrogens are a potent class of toxicants found ubiquitously in our environment as a result of their wide range use in commercial and industrial products including pesticides, plastics, and pharmaceuticals (Fenske, Maack, Schäfers, \& Segner, 2005; Ropero et al., 2006). Environmental estrogens present a significant health concern as numerous studies have demonstrated synthetic estrogens modulate and/or disrupt a variety of animal systems (Hecker \& Hollert, 2011). Such studies demonstrating physiological and developmental dysfunction on both aquatic and terrestrial vertebrate species within environmentally relevant concentrations include: reduced sperm production, altered spermantogenesis, intersex and complete feminization of males, altered female oogenesis and induction of aneuploidy in both sexes (Brown et al., 2008; Hunt et al., 2003; Kidd et al., 2007; Schultz, Skillman, Nicolas, Cyr, \& Nagler, 2003; Takahashi \& Oishi, 2006; Tyler \& Jobling, 2008).

While naturally occurring estrogens bind to estrogen receptors (ERs) and elicit a complex range of cellular actions through membrane, nuclear, and mitochondrial signaling pathways, the capacity of synthetic estrogens to bind to ERs and initiate an estrogenic response is subject to the chemical structure of the foreign estrogen and ER binding affinities in the target organs (Menuet et al., 2004). The most commonly used synthetic estrogen, 17- $\alpha$ ethynylestradiol (EE2), is found in most contraceptive products and hormone replacement therapies. EE2 has been demonstrated to be more potent than 
the endogenous estrogen, estradiol-17 $\beta$ (E2), in many fish species with a greater estimated binding affinity (Shyu et al., 2011).

Mitochondria as targets of environmental toxicants is gaining increased recognition (Meyer et al., 2013). Assessing the impact of synthetic estrogens on mitochondrial integrity is significant given the import of ERs into the mitochondria (mtERs) modulates estrogenic activity within the mitochondria and coordinates mitochondria-nuclear regulatory cross-talk. The importance of mitochondria cannot be understated as they are vital subcellular organelles key to all aspects of development, metabolism and growth (Nunnari \& Suomalainen, 2012; Youle et al., 2012). They are responsible for cellular life-death decisions and also play critical roles in other physiological processes (Cheng \& Ristow, 2013).

One significant factor in mitochondrial function is their genetic material, mitochondrial DNA (mtDNA) with all mitochondria having multiple copies of the genome within each organelle (Falk et al., 2014). MtDNA can have increased susceptibility to environmental genotoxins as a result of their physical location near reactive oxygen species (ROS) production sites, reduced protein packing and reduced repair capacity. Mutations within the mtDNA are known to cause a wide range of physiological disorders including: neurodegenerative disorders, cardiomyopathies, metabolic syndromes, cancer, obesity and premature cell senescence (Goldstein, Bhatia, \& Vento, 2013; Schapira, 2006; Wallace \& Chalkia, 2016). Disease phenotype and severity is often dependent on the proportion of wild type and mutant mtDNA within an individual, or mtDNA heteroplasmy. While many mitochondrial related disease states are 
strictly hereditarily acquired, to date no studies have assessed the potential for induced heteroplasmy as a result of environmental estrogen exposure.

The following study is a preliminary investigation assessing the effects of shortterm EE2 exposure on mtDNA stability in sexually mature male isogenic rainbow trout.

\section{Methods}

The following was done at the Pacific Northwest National Laboratory in accordance to the guidelines established by the Institutional Animal Care and Use Committee (IACUC). Male isogenic rainbow trout (O. mykiss) hybrid clones (OSU X Swanson) were obtained from Washington State University and reared to 32 months of age in circular 1,400-L tanks under pass flow-through freshwater system with in-flow rates between 15-20L/min (Young \& Wheeler, 1992). Water was sourced from Battelle Marine Science Laboratory's artesian well $($ depth $=134 \mathrm{~m})$ in Sequim Washington and aerated before transfer to holding tanks. Tank quality parameters were routinely measured in all tanks and include: temperature (mean: $11.2^{\circ} \mathrm{C}$; range: $10.9-11.4{ }^{\circ} \mathrm{C}$ ), dissolved oxygen (>9 mg/L) and $\mathrm{pH}$ (mean 7.9; range: $\mathrm{pH} 7.8-8.1$ ). Trout were fed a moist 2$6 \mathrm{~mm}$ pellet feed from Vitalis Bio-Oregon Inc. (Longview, WA USA) at a ration level of approximately $0.75 \%$ body mass to encourage growth. Trout were maintained under lighting that simulated a natural photoperiod that included a 12-minute graded sunrise and sunset period.

The $17 \alpha$-ethynylestradiol was purchased from Sigma-Aldrich. All other chemicals were reagent grade. At 32 months of age, trout were exposed to $17 \alpha-$ ethynylestradiol using a continuous flow protocol for fifty (50) days. Exposure solutions 
were prepared and added slowly using a peristaltic pump. Exposure tanks were equilibrated for three days prior to fish exposure. Trout were exposed to a nominal EE2 exposure level of 10ng/L. Control trout were unexposed to EE2 but handled and maintained as those under treated fish conditions.

After fifty days exposure, trout were euthanized using MS-222 by anesthetic overdose. Livers were excised immediately following death and rinsed with cold buffer solution described by Frezza (Frezza, Cipolat, \& Scorrano, 2007).

Liver tissue was homogenized using glass Teflon homogenizers performed at $4^{\circ} \mathrm{C}$ to minimize damage caused by phospholipases and proteases. A two-phase sucrose gradient differential centrifugation to enrich for functional mitochondria was performed. The first phase isolated cellular debris through low speed centrifugation while the second isolated mitochondria through high speed centrifugation (Frezza et al., 2007). Mitochondrial DNA was extracted from the pellet using DNeasy Blood \& Tissue Kit (QIAGEN) following standard tissue protocols.

Nucleic acid quantification was performed using NanoDrop 2000 UV-Vis Spectrophotometer. Using two mitochondrial specific primers and one nuclear specific primer, we assessed the proportion of mtDNA to nuclear DNA. Genes were amplified in $10 \mu \mathrm{L}$ reaction mixtures (Taq DNA polymerase, forward and reserve primers and BSA) containing: $1 \mu \mathrm{L}$ DNA template, concentration range $55-90 \mathrm{ng} / \mu \mathrm{L})$.

Extracted DNA was sent to Oregon Health and Science University's Massively Parallel Sequencing Shared Resource (MPSSR) for library preparation, barcoding, and sequencing. Sequencing was performed using the Illumina HiSeq 2000 Sequencer. 
Using Bowtie 2 v2.1.0, we mapped sequence reads against trout mitochondria reference genome (accession L29771) using default indexing parameters. Raw paired-end sequence data were mapped to each index using default alignment parameters in Bowtie 2 v2.1.0. Using Bowtie2 SAMtools we generated a binary BAM format, sorted the BAM file and ran mpileup to produce a format of each line representing a genomic position. A Python script was used to convert to data files which were accessible for further analysis of frequency of nucleotides diversity per site.

\section{Results and Conclusions}

We used PCR amplification to assess for genomic DNA contamination and verify enrichment of mtDNA content post organelle isolation on one EE2 treated sample, 107 and one control sample, 105.1 (Figure 3) against a test sample of total Swanson Clone DNA. Mitochondrial enrichment proved highly successful with little to no detectable nuclear DNA present in amplification.

Three fish treated at 10ng EE2/L and three untreated controls were used for comparison. We achieved an overall coverage of $8000 \mathrm{x}$ across all samples sequenced. We defined heteroplasmy as the second most common base variant per site across the mitochondrial genome. A sequencing error rate of 0.0004 or $0.04 \%$ was determined by comparing sequencing replicates of one control sample through the difference between the absolute value of heteroplasmic frequency between each site and determining the average across the genome. A linear regression comparing heteroplasmy between sequencing replicates demonstrated high consistency in same lane sequencing as seen with a $\mathrm{R}^{2}$ of 0.9939 (Figure 4 ). 
We established a conservative threshold of heteroplasmic calls to be $\geq 0.25 \%$ or $5 \mathrm{x}$ our sequencing error rate. Heteroplasmic calls were not found to be statistically significant between treated and control fish (Figure 5) although there was an increase in the number of heteroplasmic sites in almost all EE2 treated fish compared to the control (Table 1). Out of all EE2 treated fish, the lowest number of sites considered heteroplasmic is slightly greater than the highest number of sites in the control fish. Using UCSC Genome Browser to map heterplasmic sites to the mitochondrial genome, and observed site specific mutations in EE2 treated fish compared to the control (Figure $6)$.

We found consistency between sequencing replicates demonstrating low sequencing error rate when using Illumina Next-Generation Sequencing technology. Although we achieved high depth of coverage, 8000x in our approach for mtDNA enrichment, we desired greater coverage for our subsequent experimental treatments to ensure greater confidence in determining heteroplasmic calls. Even with our low sample size, $n=3$ per group, we see site specific mutations and an overall increase in heteroplasmy between treated and untreated fish. This preliminary study provided a base for improving technique and supports continued investigation of assessing whether treatment of EE2 can increase heteroplasmic frequency in mtDNA. 


\section{Figures and tables}

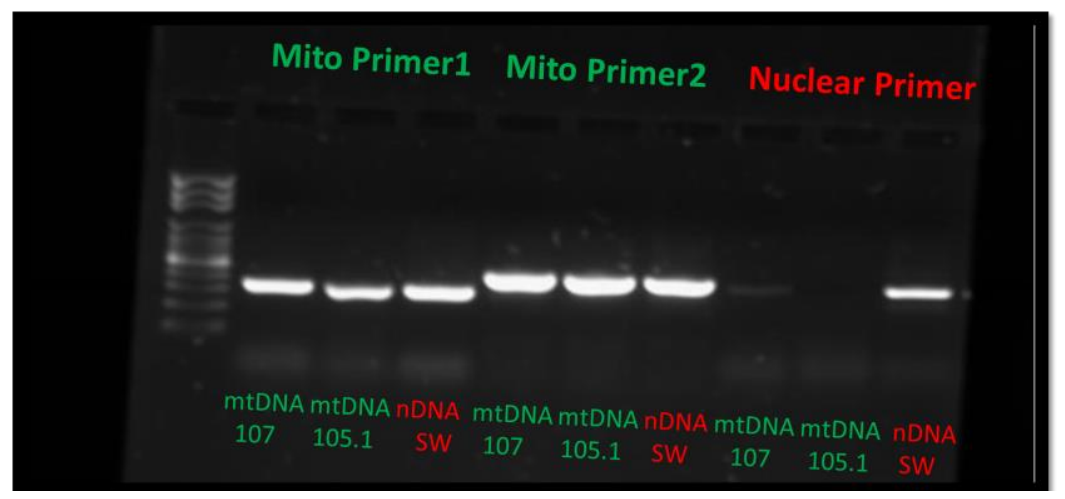

Figure 3: PCR amplification assessing genomic DNA contamination after mitochondrial organelle isolation in EE2 treated trout sample, 107 and control sample, 105.1 against test sample SW containing total DNA.

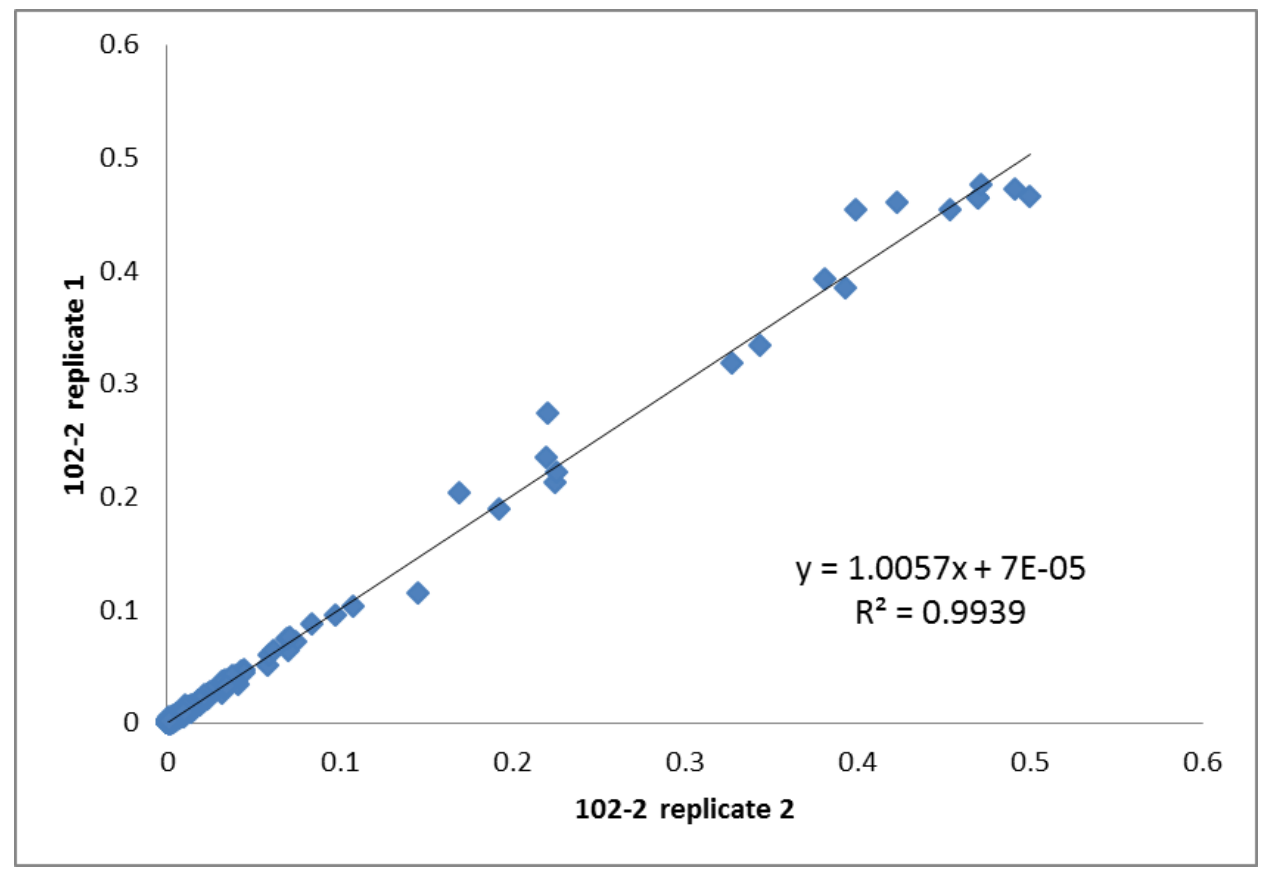

Figure 4: Linear regression of heteroplasmy between sequencing replicates in trout control sample. 


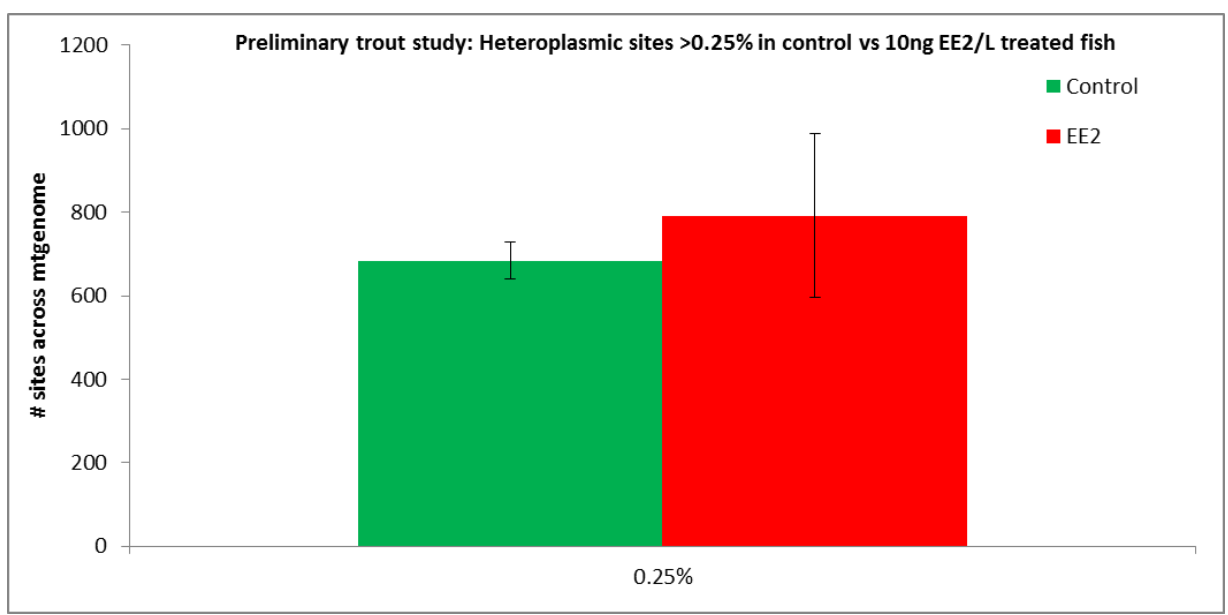

Figure 5: Average number of heteroplasmic sites $\geq 0.25 \%$ between EE2 treated (red) and control (green) trout $(\mathrm{p}>0.05)$.

Table 1: Heteroplasmic sites $\geq 0.25 \%$ in EE2 treated vs control trout samples

\begin{tabular}{l|r}
\hline Sample ID & $\begin{array}{r}\text { Heteroplasmic } \\
\text { sites } \mathbf{\geq 0 . 2 5 \%}\end{array}$ \\
\hline cntrl102-2_rep1 & 644 \\
\hline cntrl102-2_rep2 & 707 \\
\hline cntrl105-1 & 734 \\
\hline cntrl105-2 & 652 \\
\hline EE2 107 & 710 \\
\hline EE2 104-1 & 1015 \\
\hline EE2 104-2 & 650
\end{tabular}




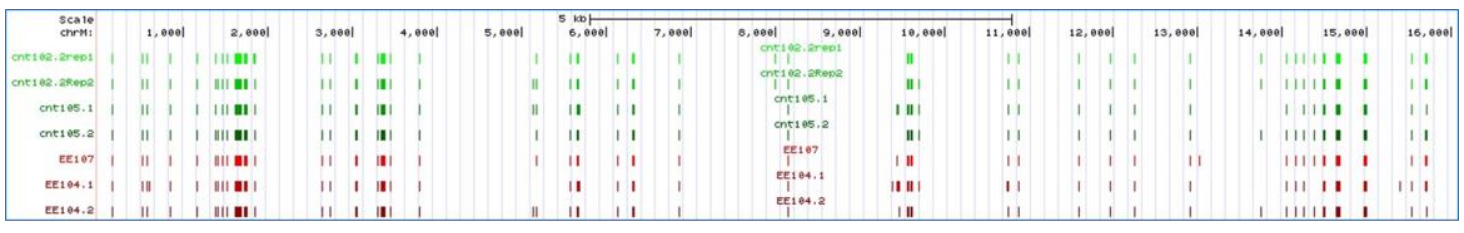

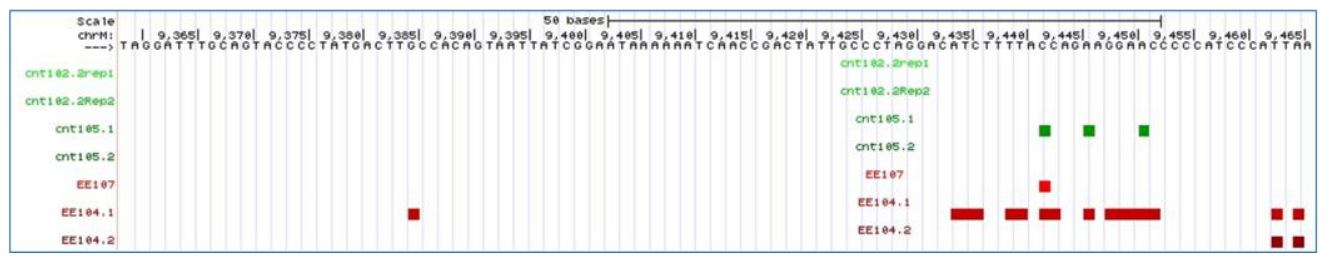

Figure 6: Mutational frequencies $\geq 0.25 \%$ viewed in UCSC genome browser in control (green) and EE2 treated (red) trout across the whole mitochondrial genome (top) and zoomed into positions 9-9.5K. 


\section{Chapter III: Acute treatment study and baseline comparisons \\ Introduction}

Environmental estrogens are a potent class of toxicants found ubiquitously in our environment as a result of their wide range of uses in commercial and industrial products including pesticides, plastics, and pharmaceuticals (Fenske et al., 2005; Ropero et al., 2006). Environmental estrogens present a significant health concern as numerous studies have demonstrated synthetic estrogens modulate and/or disrupt a variety of animal systems (Hecker \& Hollert, 2011). Such studies demonstrating physiological and developmental dysfunction on both aquatic and terrestrial vertebrate species within environmentally relevant concentrations include: reduced sperm production, altered spermantogenesis, intersex, complete feminization of males, altered female oogenesis and induction of aneuploidy in both sexes (Brown et al., 2008; Hunt et al., 2003; Kidd et al., 2007; Schultz et al., 2003; Takahashi \& Oishi, 2006; Tyler \& Jobling, 2008).

While naturally occurring estrogens bind to estrogen receptors (ERs) and elicit a complex range of cellular actions through membrane, nuclear, and mitochondrial signaling pathways, the capacity of synthetic estrogens to bind with ERs and initiate an estrogenic response is subject to the chemical structure of the foreign estrogen and the ER binding affinities in the target organs (Menuet et al., 2004). The most commonly used synthetic estrogen, 17 $\alpha$-ethynylestradiol (EE2), is found in the majority of contraceptive products and hormone replacement therapies. EE2 has been demonstrated to be more potent than endogenous estrogen, estradiol-17 $\beta$ (E2), in many fish species with a greater estimated binding affinity (Shyu et al., 2011). 
Mitochondria as targets of environmental toxicants is gaining increased recognition (Meyer et al., 2013). Assessing the impact of synthetic estrogens on mitochondrial integrity is significant given the import of ERs into the mitochondria (mtERs) modulates estrogenic activity within the mitochondria and coordinates mitochondria-nuclear regulatory cross-talk. The importance of mitochondria cannot be understated as they are vital subcellular organelles key to all aspects of development, metabolism and growth (Nunnari \& Suomalainen, 2012; Youle et al., 2012). They are responsible for cellular life-death decisions and also play critical roles in other physiological processes (Cheng \& Ristow, 2013).

One significant factor in mitochondrial function is their genetic material, mitochondrial DNA (mtDNA) with all mitochondria having multiple copies the genome (Falk et al., 2014). MtDNA can have increased susceptibility to environmental genotoxins as a result of their physical location near reactive oxygen species (ROS) production sites, reduced protein packing and reduced repair capacity. Mutations within the mtDNA are known to cause a wide range of physiological disorders including: neurodegenerative disorders, cardiomyopathies, metabolic syndromes, cancer, obesity and premature cell senescence (Goldstein et al., 2013; Schapira, 2006; Wallace \& Chalkia, 2016). Disease phenotype and severity is often dependent on the proportion of wild type and mutant mtDNA within an individual, or mtDNA heteroplasmy. While many mitochondrial related disease states are hereditarily induced, to date no studies have assessed the potential for induced heteroplasmy as a result of environmental estrogen exposure. 
The goal of this study was to examine the effects of EE2 on mitochondrial genome stability after acute exposure to zebrafish during the bio-energetically demanding period of sexual differentiation. In addition, we assessed the effects of EE2 on the proportion of females between untreated fish and treatment groups, tracking survivorship between environmentally relevant treatment concentrations. Lastly, because mitochondria is maternally inherited, we used the maternal lines for comparison of full-sib treatment groups as well as fish for baseline comparison from each strain to assess standing rates of heteroplasmy.

\section{Methods}

All fish were maintained in accordance to guidelines established by the Institutional Animal Care and Use Committee (IACUC) of Portland State University. Adult male and female zebrafish, of the three common laboratory strains $\mathrm{AB}, \mathrm{Tu}$, and WIK, less than one year of age were obtained from the Zebrafish International Resource Center (ZIRC) for use as either baseline analysis fish, maternal lines or for the generation of full-sib treatment groups. Fish were housed in 2.8-liter baffled flow-through tanks at a ratio of 1 male: 1 female with a stocking density of 10 or less fish per $2.8 \mathrm{~L}$ tank in the Brown Zebrafish Aquatics Facility at Portland State University. Fish were maintained on the Aquaneering Modular System with 4-stage central filtration providing continuous flow-through of recirculating water with a twice daily automated $10 \%$ water change. This system monitors and maintains parameters within a narrow range to ensure environmental effects are negligible. The aquatic facility utilizes a photo period of $15 \mathrm{~h}$ 
light: 9 hr dark. Adult fish were fed two times a day ad libitum with larvae power and live Artemia salina (brine shrimp).

Sexually mature zebrafish used for generation of treatment fish were set up as breeding pairs (1 male:1 female) in Aquaneering Crossing Tanks with dividers to separate each pair. The following morning, dividers were removed and zebrafish were allowed to spawn for up to 6 hours. Spawning occurred within the aquatic facility under standard room and system parameters.

Embryos were collected, rinsed with embryo media (Cold Harbor Springs 60x recipe), and kept at a stocking density of $\leq 50$ embryos per petri dish in media at $28^{\circ} \mathrm{C}$ for 5 days. At 5 days post fertilization (dpf) hatched larvae were transferred to static tanks containing $\sim 250 \mathrm{~mL}$ media with water changes performed daily. Larvae were fed concentrated rotifers twice daily ad libitum. At 9dpf larvae began transition to live brine shrimp in addition to rotifers. At 11dpf larvae were transferred to the main system and placed under a slow drip of $\sim 1$ drop/second. Larval powder was introduced at 14 days and fish were transitioned to an adult feeding schedule at 3 weeks of ago. Juveniles were raised under these conditions until 6 weeks of age for exposure initiation.

The exposure system (Figure 7) was designed and built in house. 20-gallon fish tanks were used as common mixing tanks ( $\mathrm{x} 4$ treatment groups) and 1 gallon fish bowls were used as treatments tanks to house respective pair/strain/treatment type ( 2 pairs per strain, 3 strains). Using PVC piping and a centrifugal pump, pre-mixed exposure water was routed circularly with an inflow ball valve into 1 gallon fish bowls. Each exposure tank had an outflow valve routed into a common waste basin which was pumped through 
a triple filtration system before flowing into the common building drainage and city wastewater system. Common mixing tanks were spiked with EE2 or methanol (for control) and allowed to equilibrate for 24 hours prior to transfer of juvenile fish.

The test compound 17 $\alpha$-ethynylestradiol (EE2) was obtained from Sigma-Aldrich ( $\geq 98 \%$ purity grade). EE2 was solubilized in $100 \%$ methanol at a ratio of $1 \mathrm{mg} / 1 \mathrm{~mL}$. A final working concentration of $10 \mathrm{ng} \mathrm{EE} 2 / \mu \mathrm{L}$ in $10 \%$ methanol was prepared as the final exposure working stock for spiking treatment water. Control fish were exposed to methanol at the highest EE2 treatment concentration, $9.5 \mu \mathrm{L}$ methanol/gallon tank water or $\leq 0.00025 \%$.

At 6-weeks of age, following sexual differentiation, juvenile zebrafish (Danio rerio) from strains $\mathrm{AB}, \mathrm{Tu}$, and WIK were exposed for 6 weeks to nominal values of 10ng EE2/L, 100ng EE2/L, and 250ng EE2/L (Figure 8). Control fish were exposed to $\leq 0.00025 \%$ methanol, equivalent to the greatest amount of methanol used on the 250ng EE2/L treated fish. Two sets of full-sib juveniles were used for each strain totaling six families per treatment type.

Two acute treatment series were performed (acute exposure (1) and acute exposure (2)). During the initial trial, an immediate die-off of juvenile fish was observed and discovered to be a result of low water conductivity, $0.02 \mathrm{ppt}$. Initial common mixing tank water was sourced from the main system's fresh water sump after dechlorination which reduced ions levels below acceptable levels. Hours into the initial exposure, $2 \mathrm{ml}$ $\mathrm{NaCl} /$ gallon was added to common mixing tanks. Thereafter, each common mixing tank fill included $2 \mathrm{ml} \mathrm{NaCl}$ per gallon of water. We had a target of 20 individuals per pair and 
treatment. For the duration of the treatment, exposure water was semi-static with daily 30-second flushes per bowl.

Baseline fish were also tested to establish nominal background rates of heteroplasmy between strains. Ten adult zebrafish (5 females and 5 males) from each of the three strains were raised under standard rearing conditions to 2 years of age, as described above. Assessment of these fish will ensure that baseline heteroplasmic frequencies are similar between untreated and methanol only treated individuals as well as for comparison of between strain variations.

Fish were euthanized using MS-222 (Sigma-Aldrich) at a concentration of $300 \mathrm{mg} / \mathrm{L}$ water at $\mathrm{pH} 8$ for $\geq 15$ minutes. Weight and sex was recorded prior to liver extraction. Liver tissue was collected from zebrafish by surgical manipulation under a stereoscopic microscope (Leica Microsystems) and stored at $-20^{\circ} \mathrm{C}$ until further use.

Total DNA was isolated from liver tissue using QIAGEN DNeasy Blood \& Tissue Kit. Nucleic acid quantification was performed using NanoDrop 2000 UV-Vis Spectrophotometer. To assess for mtDNA in total DNA, two primer pairs of mitochondrial encoded protein subunits for cytochrome $b$ and one primer pair of nuclear encoded protein subunit for rhodopsin were used. Genes were amplified in $10 \mu \mathrm{L}$ reaction mixtures (Taq DNA polymerase, forward and reserve primers and BSA) containing: $1 \mu \mathrm{L}$ DNA template, concentration range 55-90ng/ $\mu \mathrm{L}$.

We used custom designed Agilent SureSelect ${ }^{\mathrm{XT}}$ Target Enrichment Kit for Illumina Multiplex Sequencing to capture the following 5 genomic regions of interest: mitochondrial genome (1-16596bp), chromosome 19 (9838621-9841031bp), 
chromosome 22 (17417393-17419398bp), chromosome 22 (17422326-17422970bp) and chromosome 7 (31329397-31333402bp). Four probes were designed for regions of the nuclear genome, three for mitochondrial encoding nuclear genes (ndufv1 - NADH dehydrogenase of complex I, and two regions ATPAF1 - assembly factor of ATP synthase) and one for a non-mitochondrial related nuclear encoded gene (isl2b - insulin gene enhancer protein).

Paired-end Illumina libraries were captured in accordance to the SureSelect protocol with the following modifications. Input DNA and buffer for fragmentation was doubled to $6 \mu \mathrm{g}$ DNA into $260 \mu \mathrm{L}$ total volume 1x TE buffer. Sonication was performed for a median fragment target size of 150-200bp on Cole-Parmer Ultrasonic Processor at $60 \%$ amplitude, for a total of 4.5 minutes sonication time with on-off intervals of 9-30 seconds on and 8-30 seconds off. For the first sample purification step using Agencourt AMPure XP beads, input bead volume and concentration of sheared DNA library was doubled. The products were end-repaired, 3'non-template A's were added, and pair-end adapters were ligated. Ligated libraries were PCR amplified for 6 cycles.

DNA libraries were hybridized to custom biotinylated probes with subsequent immobilization on magnetic beads. After elution of the captured target regions, libraries were amplified with 1 of 16 unique index primer tags for each sample in accordance to intra or inter lane sequencing design.

Quantification and quality assessment of indexed-captured libraries was done using Agilent 2100 Bioanalyzer and qPRC at Oregon Health and Science University's Massively Parallel Sequencing Shared Resource (MPSSR). Index-tagged libraries were 
pooled for multiplexed sequencing. Using the Illumina HiSeq 2000 Sequencer, 100-bp paired-end reads were generated for each sample.

Using Bowtie 2 v2.1.0, we prepared three indexes for reference using default indexing parameters. For the zebrafish mtDNA reference, GRCz10 (GB GCA_000002035.3) was used to prepare the first index (mtDNAindex). Because the mtgenome is a circular molecule, we prepared a 936bp long concatenated index (concatenated_mtDNA_index) by manually clipping the first 456 bp and last 480 bp locations and wrapping them to close the genome. A third index was created using the zebrafish reference Zv9 (GCA_000002035.2) by selecting out the nuclear target regions per probe design. The GRCz10 reference release is an improved assembly of Zv9 which was a preliminary assembly; however MT assemblies are identical within the two versions. The $\mathrm{Zv} 9$ assembly was used as an index to our nuclear probes based on the design date of our probes being prior to the release of the revised assembly.

Raw paired-end sequence data were mapped to each index using default alignment parameters in Bowtie 2 v2.1.0. Using Bowtie2 SAMtools we generated a binary BAM format, sorted the BAM file and ran mpileup to produce a format of each line representing a genomic position. A Python script was used to convert to data files which were accessible for further analysis of frequency of nucleotides per site.

Exposure water samples $(400 \mathrm{~mL})$ were collected weekly throughout the duration of the exposure. Control water samples $(400 \mathrm{~mL})$ were collected at the half-way and final week time points of the exposure duration. Both exposure water and control samples were immediately spiked with internal standard (50ng IS/L). Samples were stored at - 
$20^{\circ} \mathrm{C}$ until further analysis. Samples were thawed to room temperature 24 hours prior to sample preparation for quantification.

Compounds used for LC-MS analysis include the following: triethylamine (TEA) and 17a-ethynylestradiol (EE2) were obtained from Sigma-Aldrich. Acetonitrile (ACN) and water (LC-MS grade) were from Burdick and Jackson (Muskegon, MI). 17ßEstradiol-16, 16, 17-d3 (E2D3) was from Toronto Research Chemicals Inc.

Quality control samples were generated at concentrations of 10, 100, and 250ng EE2/L using dilutions of authentic standard in methanol as in those used to spike common mixing tanks. Calibrators were generated to cover the concentration range of EE2 in mixing tank water from 1ng-250ng EE2/L using dilutions of authentic standard dried and reconstituted in mobile phase.

D3 tagged estradiol (E2D3) was used as an internal standard (20 $\mu \mathrm{L}$ at $1 \mathrm{ng} / \mu \mathrm{L})$ in $100 \%$ methanol added to QC samples, samples and calibrants. $400 \mathrm{~mL}$ samples or QC samples were passed through a $3 \mathrm{~mL}$ Oasis HLB SPE cartridge (Waters) conditioned with $3 \mathrm{~mL}$ of methanol using a vacuum manifold. Analytes were eluted using $2 \mathrm{~mL}$ methanol, dried under a vacuum, and reconstituted in $100 \mu \mathrm{L}$ starting mobile phase.

LC-ESI-MS analyses were performed using a high-resolution $(30,000)$ Thermo LTQ-Orbitrap Discovery hybrid mass spectrometry instrument (San Jose, CA) equipped with an electrospray ionization source operating in the negative mode. The ESI interface using the following settings: sheath gas flow rate 40 (arbitrary units), aux gas flow rate 15 (arbitrary units), spray voltage $2 \mathrm{kV}$, capillary temperature $250 \mathrm{C}$, capillary voltage $-17 \mathrm{~V}$, and tube lens voltage $-85 \mathrm{~V}$. The Orbitrap was externally calibrated prior to data 
acquisition allowing accurate mass measurements for $[\mathrm{M}+\mathrm{H}]^{+}$or $[\mathrm{M}-\mathrm{H}]^{-}$ions to be obtained to within $4 \mathrm{ppm}$.

The Orbitrap was coupled to a Thermo Accela HPLC system. EE2 was resolved using a Kinetex C18 (4.6x50mm, $2.1 \mathrm{~mm}$ i.d., 2.5uL particle size) HPLC column with guard cartridge (Phenomenex; Torrance, CA). Mobile phases consisted of $0.025 \%$ TEA in HPLC grade water (A), 0.025\% TEA in 95:5 methanol:water (B). The gradient mobile phase was delivered at a flow rate of $0.3 \mathrm{~mL} / \mathrm{min}$ and the TEA:water mobile phase.

Start conditions were $5 \% \mathrm{~B}$, increased to $40 \%$ B over 2 minutes, increased to $45 \%$ B over the next 5 minutes, $100 \%$ B over the next three minutes. Column was washed at $100 \% \mathrm{~B}$ for 1 minute and back to $5 \%$ B for 5 minutes. The column temperature was kept at $25^{\circ} \mathrm{C}$ using a thermostatic column oven. The sample injection volume was $50 \mu \mathrm{L}$.

\section{Results and Conclusions}

We exposed 6 groups of full-sib offspring from 3 strains of zebrafish: $\mathrm{AB}, \mathrm{Tu}$, and WIK, across 4 treatment categories: 10ng, 100ng, 250ng EE2/L and control (methanol $\leq 0.00025 \%$ ) to assess the effects of EE2 on mitochondrial genome stability during sexual differentiation, 6 weeks of age. We observed lethality among 100ng and 250ng EE2/L treatment groups limiting our sequencing comparisons to the control and 10ng EE2/L treatment group. We used Illumina next generation sequencing technology for sequencing ultra-pure mtDNA after target enrichment from liver tissue of acute exposure (2) study groups (Table 2): (a) 10ng EE2/L and control (at $\leq 0.00025 \%$ methanol) for 6 weeks, from three strains of zebrafish $\mathrm{AB}, \mathrm{Tu}$, and WIK, using two separate maternal/paternal (1-1ab, 8-3ab, 2-1tu, 7-3tu, 1-1wik, 1-2wik) lines per strain; (b) the maternal line, as above, from 
each pair used to generate full-sib offspring for treatments; (c) 10 baseline fish (5 males and 5 females) over 2 years of age from each strain; and lastly, (d) five replicate samples (not in table) from each representative group to correct for sequencing error.

Actual start counts consisted of 13-23 acute exposure (1) and 18-21 acute exposure (2) due to natural die off during the first 6 weeks of rearing in untreated system. We were interested in comparing the survivorship of each treatment type (10ng, 100ng, and 250ng EE2/L) to control $(\leq 0.00025 \%$ methanol) using three zebrafish strains (AB, Tu, and WIK) (Figure 9-12, top from acute exposure (1) and bottom from acute exposure (2)). During the initial acute exposure (1), we had 2 issues: (a) initial counts were lost because of recording errors, (b) rapid initial die-off within all treatment and control groups predominantly in strain Tu. Survival curves from acute exposures (1) which begin past day 0 show the first day of accurate counts for the associated strain and treatment type recorded (e.g. acute exposure (1) 250ng EE2/L, TU-1 begins at $76 \%$ on day 14 , meaning between day 0-14 seven individuals were lost without record of individuals lost per day). The rapid die-off was quickly discovered to be the result of low conductivity in sourced water as described in the methods. The general trend seen from the acute exposure (1) after initial die-off and start of survival counts was found to be $95 \%$ or greater survival in the control and 10ng EE2/L groups across all three zebrafish strains and 50\% survival or less across all three strains of 100 and 250ng EE2/L treatment groups.

Survivorship for acute exposure (2) series had similar patterns to exposure (1). Greater than 95\% survival was seen in all pairs and strains in the control group of acute 
exposure (2) with the exception of Tu pair 2.1; however, $30 \%$ loss in this pair/strain occurred within the first 2 days of the exposure start date and remaining die-off, 5\% occurred within the first 4 days of exposure start which was likely associated with acclimation sensitivity following tank transfer. Post-day 4, Tu 2.1 had 100\% survival. All pair and strains within 10ng EE2/L treatment had greater than $90 \%$ survival lacking any indication of lethality at this concentration. As seen in acute exposure (1), both 100ng and 250ng EE2/L were found to be lethal in all 6 pairs used with the exception of WIK 1.1 at 100ng EE2/L which concluded the treatment at 55\% survival. Phenotypically, surviving individuals in 100ng and 250ng EE2/L treatment groups were greatly reduced in size and weight, had a great degree of ascites, raised scales, and in some cases bulging eyes, and enlarged hearts suggesting severe impairment of growth and developmental pathways and inhibition of normal organ function.

Until 2013, sexual determination and differentiation pathways in zebrafish were considered mostly unknown. Liew and Orban (2013) did an overview of zebrafish sex in two strains of zebrafish (AB and Singapore based wildtype) where they identified three major developmental pathways associated with gonad differentiation but concluded further analysis is necessary to elucidate this process. With sexual differentiation occurring 21-30 dpf (Liew, Bartfai, Lim, Sreenivasan, \& Siegfried, 2012), and our exposures beginning following sexual differentiation, 42 days of age, we were interested in evaluating sex ratios in EE2 treated compared to control fish (Liew et al., 2012; Liew, 2013). 
The proportion of females post-42 day acute exposure (2) between control and treatment (Figure 13) was not statistically significant; however, an increase was observed in the proportion of females in 10ng EE2/L compared to controls. In the 100ng and 250ng EE2/L treatment groups of each strain and pair, either $0 \%$ survival occurred or among the few surviving individuals $(\leq 55 \%)$, gonad type could not be determined from gross dissection. This appeared to be due to stunted growth and severe fluid within the body cavity.

Calibration curves were generated by performing a least-square linear regression for peak area ratios (EE2 analyte/E2D3 internal standard) plotted against specified calibrant concentration in water (ng/L). The lower limit of quantification (LLOQ) was determined as the lowest concentration for which the signal-to-noise $(\mathrm{S} / \mathrm{N})$ ratio was $\geq 5$ and the within- and between-day reproducibility of the peak area was $\leq 20 \%$ relative standard deviation (RSD). For all other calibrators the between and within-run precision (RSD) for calculated concentration across the range 1-250ng/L was $\leq 15 \%$ and accuracy was within $\pm 15 \%$ (detailed precision and accuracy information for calibrators and low and high QCs provided in Table 3).

The goal of these experiments was to validate that the measured concentration of common tank exposure water to treatment groups was within +/- 25ng EE2/L of spiked concentration. The data we generated supported this (Table 4), calculated mean concentrations of EE2 in mixing tank samples. In addition, EE2 was not detected in the control samples taken from the half-way and end-of-exposure time points of acute exposure (2) showing control fish were not exposed to EE2. 
Total DNA was isolated from liver tissue and quantified as described in the methods with the following two exceptions: (a) the maternal fish from 2-1tu (pair 2-1, strain $\mathrm{Tu}$ ) died of natural causes while on the main aquatic system and upon retrieval, adequate DNA for sequencing preparation was not successfully extracted from either liver tissue or through fin clip attempts, (b) maternal fish from 8-3ab (pair 8-3, strain ab) died of natural causes on the main aquatic system; however, adequate DNA was extracted through superior anal fin clip opposed to liver extraction. Because mitochondria are maternally inherited the loss of 2-1tu maternal DNA resulted in the exclusion of 2-1tu control (M1-M6) and treated fish (10.1-10.6) from the analysis of sequence data.

All samples were assessed through gel electrophoresis post-sonication to ensure target fragment size of 150-200bp or larger was achieved (Figure 14). During the initial stages of working through the protocol, select samples were assessed for quality control post-sonication and post-AMPure bead clean-up 1 but prior to library preparation using Agilent 2100 BioAnalyzer (Figure 15). Samples were quantified using $1 \mu 1$ DNA at each step of the library preparation protocol to ensure adequate DNA content was being carried through the procedure.

Using target enrichment we achieved an average sequence coverage of $>88,900 x$ across our entire sequencing project (Tables 5-9). Over $70 \%$ of samples sequenced with an average coverage $>90,000 x$. The lowest coverage within samples was $>1000 x$ at sites profiled within the D-loop (between 1-950bp). Overlaid sequence coverage of replicate samples demonstrates coverage across the mitochondrial genome and specific regions of high vs low coverage. These replicates demonstrate that seen across all sequenced 
samples. Overall read counts and alignment to the mitochondrial references genome ranged from 6-75million reads and $70.17-98.85 \%$ (table 10-14). Of these, $92 \%$ of sample's reads mapped at $\geq 90 \%$ and $81 \%$ of sample's reads mapped $\geq 95 \%$ to the reference mtgenome.

We achieved ultra-high coverage by using target enrichment to select for pure mtDNA; however, sequencing error still presents as a challenge of modern sequencing technologies. To establish a robust sequencing error rate we used a conservative strategy of 5 replicate sample comparison, two within the same lane and three between lanes. To ensure we accurately captured an error rate representative of all sample types, we sequenced replicates from each group within our study: 1-methanol control, 2-10ng EE2/L treated fish, 2-baseline fish and of these, at least one from each strain was used as a replicate as demonstrated in overlaid sequence coverage (Figure 16). In addition, we sequenced replicates of full sibs from control and treated fish as well. A linear regression performed on one of each sample and comparability of between and same lane sequencing shows consistency between samples (Figure 17).

We defined a heteroplasmic site as the number of calls for the second most common allelic variant out of all calls for that site. Our standard for determining error in each replicate sample was set by finding the heteroplasmic frequency of each site in both replicates, subtracting the absolute value of heteroplasmy at each site and finding the average across the entire mtgenome of the replicate differences (Table 15). The difference between the highest and lowest error rate found was 0.000078 or $0.0078 \%$. As there was little variability between replicates, we established our sequencing error rate to be 
0.000147 or $.0 .015 \%$, the highest error rate found out of all our replicate samples. We observed a much lower error rate compared to our preliminary investigation by using improved techniques and increase sample size.

Additionally, we profiled the top ten sites with the greatest heteroplasmic variance between replicates (Table 16). Three out of five of our replicate samples top sites of variance were entirely within non-coding D-loop of the mtgenome, the most polymorphic region of the genome. Of the two other replicates investigated with highly variable sites in encoding regions, the WIK replicate had the highest frequency at $7.6 \%$ at the top site; however, this location, 10535, was found to be strain specific and the top heteroplasmic site across all WIK samples sequenced along with the other highly variable sites listed within this strain.

Through target enrich we were able to obtain highly pure mtDNA for sequencing. Sequencing artifacts (eg, adaptor dimers) that could typically compromise actual heteroplasmic calls were filtered out through selection with AMPure bead clean-up. The addition of paired-end molecular barcodes to adaptor sequences along with at or above target fragment length increased our confidence in read quality and alignment strength of our 100bp length reads. To ensure our identification of heteroplasmic sites was the result of actual allelic variants and not an artifact, we established our heteroplasmic threshold as $5 x$ our established error rate $(0.0015 \%)$ at $0.075 \%$. All considered along with our depth of coverage, we still set a conservative threshold for resolving actual structural variants, allowing for confidence in heteroplasmic calls. Our threshold was applied to all samples and any sites with allele frequencies above were considered heteroplasmic sites 
Ameur et al. (2011) estimated point mutation frequency by looking at mutations per nucleotide and finding the median, reporting a standing frequency of 0.00013 0.00018 in wild-type mice and 0.0012 in mutator mice expressing a proof-readingdeficient mtDNA polymerase (Ameur et al., 2011). Additionally, He et al. (2010) established heteroplasmic variants $2 \mathrm{x}$ their established error rate when comparing tumor to normal cells in humans (He et al., 2010).

We sequenced mtgenomes from 5 females and 5 males from three strains of zebrafish. Using a heteroplasmic threshold of $0.075 \%$ (Figure 18), we saw no differences between standing rates of heteroplasmy between males and females within the same strain or between strains (Figure 19; left side). Additionally, we saw no differences between standing rates of heteroplasmy over $0.075 \%$ when males and females were pooled and strains were compared, AB-2077, Tu-1536, WIK-2289 sites (Figure 19; right side).

Within strain and pair comparisons of heteroplasmic sites at $>0.075 \%$ showed the standing heteroplasmic frequency of the maternal lines relative to control and treated fullsibs of that line (Figures 20-22). In each strain we see the frequency of heteroplasmy between control groups reflective of standing maternal heteroplasmy and a general increase in the 10ng EE2/L treated fish. Heteroplasmy in maternal lines (Figure 23) is consistent across strains, similar to what was seen in baseline fish comparisons.

When we compare treatment vs control of each pair/strain (Figure 24), a statistically significant difference between control and treated is seen within one pair/strain combo, 8-3ab; however although it is not statistically significant, there is a 
consistent trend across all strains and pairs demonstrating an increase in heteroplasmy between treatment and control.

Comparing pooled control fish to pooled 10ng EE2/L treated fish (Figure 25), we see a statistically significant difference between heteroplasmic variants at our determined threshold $(\mathrm{p}<0.05), 2442 \pm 439$ vs $4462 \pm 918$. In our final comparison of heteroplasmy between all groups: control, 10ng EE2/L treated, baseline and maternal (Figure 26), as before we see statistically significant differences between control vs treated but also between 10ng EE2/L treated vs baseline groups. This is not seen between baseline and control or maternal and control. There was also no difference between maternal and any other group however a sample size of only 5 fish appears to limit this comparison to the maternal group while each other had a sample size of 30 .

Within the vertebrate mtgenome, hypervariable sites exist predominantly within non-coding regions (i.e., the d-loop). Ameur et al. defined sites with single nucleotide variant frequencies $(\mathrm{SNV})>0.5 \%$ as mutational hotspots, a value also used in both mouse and human heteroplasmic studies. We found $1-30 \%$ of our heteroplasmic sites to fall in this category. By this definition, we saw no clear differences between mutational hotspots in treated vs untreated fish within the acute exposure sequencing group.

The goal of this study was to examine the effects of environmentally relevant concentrations of EE2 on three common strains of zebrafish at the life cycle stage of sexual differentiation. Our results show two out of three concentrations of EE2 (100ng and 250ng EE2/L) were lethal to all three strains of zebrafish during this stage of development. Consistent with other studies, we found exposure to EE2 during sexual 
differentiation increased the proportion of females relative to control groups; however this was not statistically significant. Given that we could only compare the lowest concentration of EE2 (at 10ng EE2/L) to the control to assess female proportion, we were limited in our comparison across multiple treatment groups.

Our sequencing results using SureSelect Target Enrichment for mtDNA selection shows ultra-high sequencing coverage with an average of 90,000x across our sequenced samples giving us confidence in our heteroplasmic calls. Our use of sequencing five replicate samples and unique lane design allowed us to establish an error rate of $0.015 \%$ and determine a conservative heteroplasmic threshold of $0.075 \%$. Using this threshold, we compared heteroplasmic calls within and between strains. Our results show an increase in heteroplasmic sites both within and between strains in 10ng EE2/L treated fish compared to control; however, the level was not statistically significant. Comparison of all treated to untreated fish shows a statistically significant difference between the groups.

The loss of two out of three of our treatment groups during exposure greatly limited our comparisons of heteroplasmy between treated and untreated fish. With our current results indicating an overall increase in heteroplasmy between 10ng EE2/L treated fish compared to control fish, continued assessment of these effects are necessary. The above assessment is important for understanding the vulnerability of the mitochondrial genome to toxicant exposure. Are higher concentrations at different life stages susceptible to similar effects? Although the exact mechanisms by which this occurs is still unknown, determining the extent of genomic changes to the mitochondrial genome after EE2 
exposure is the first step in determining whether mtDNA can be a target of xenoestrogens. If so, to what extent can these changes alter mitochondrial function?

Figures and Tables

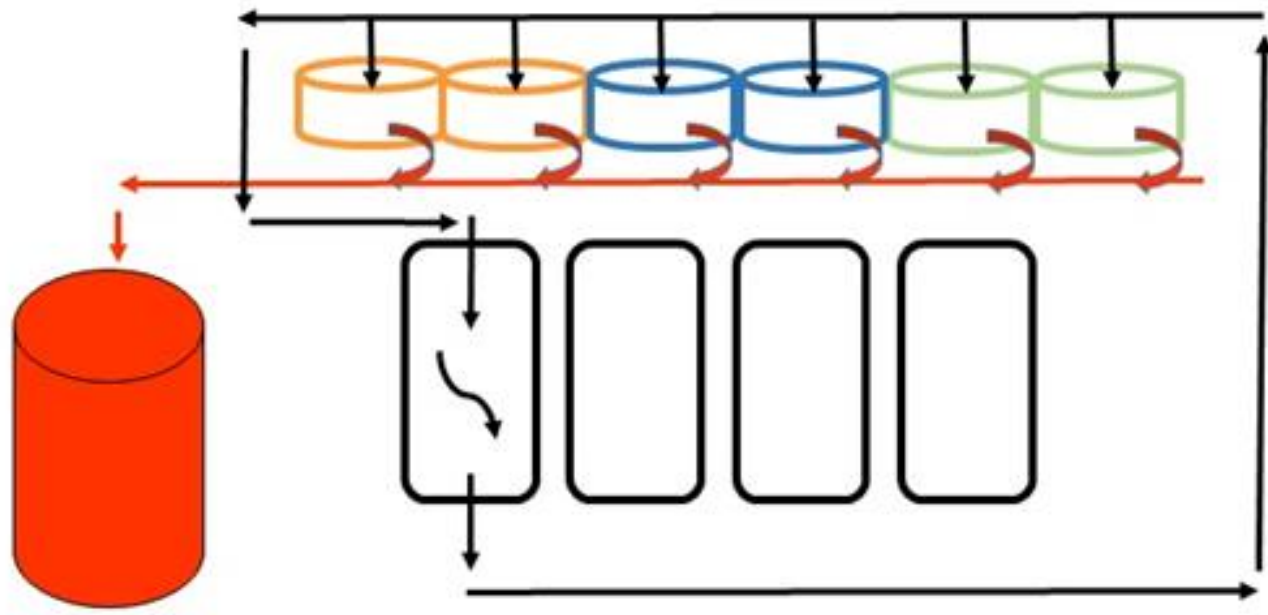

Figure 7: Diagram of exposure system used to treat zebrafish to 17- $\alpha$ ethynylestradiol (black arrows represent circular flow of exposure water, red arrows represent outflow of waste water, colored fish bowls represent three strain designations.)

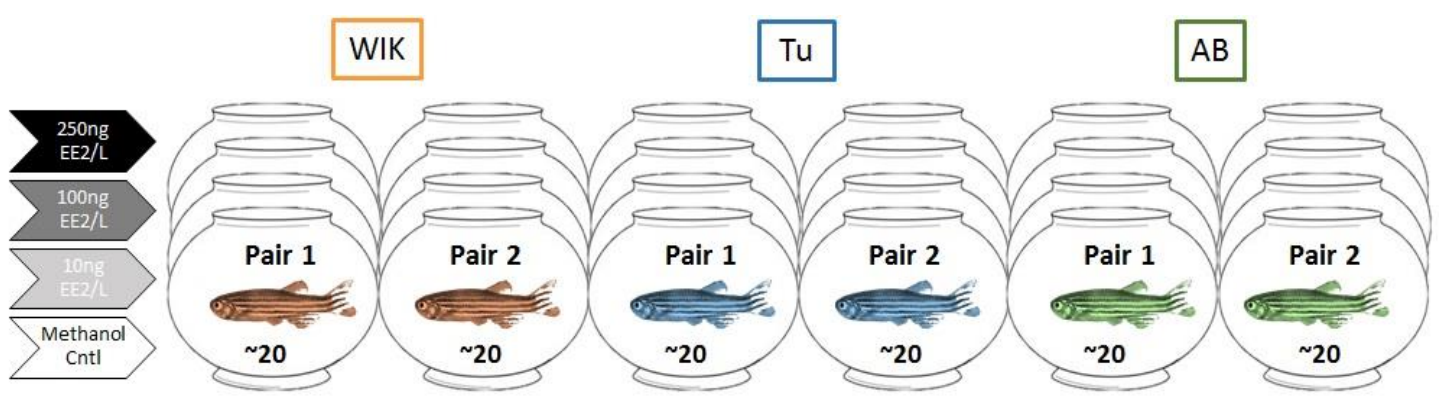

Figure 8: Acute exposure design. Three EE2 treatment groups (10, 100, 250ng EE2/L) and one methanol control group, three zebrafish strains (AB, Tu, WIK), two breeding pairs per strain to create full-sib starts. Six week exposure duration starting at 6 weeks of age. 
Table 2: Acute exposure (2) sequencing sample key

\begin{tabular}{|c|c|c|c|c|c|c|}
\hline Maternal & \multicolumn{3}{|c|}{ Treated } & \multicolumn{3}{|c|}{ Baseline } \\
\hline \multirow{2}{*}{ ID } & \multirow{2}{*}{ Full-sib ID } & $\begin{array}{c}\mathrm{MeOH} \\
\text { cntrl }\end{array}$ & \multirow{2}{*}{ \# ID } & \multirow{2}{*}{ Baseline } & \multirow{2}{*}{ ID\# } & \multirow{2}{*}{$\operatorname{sex}$} \\
\hline & & $\begin{array}{c}\text { 10ng } \\
\text { EE2/L }\end{array}$ & & & & \\
\hline \multirow{2}{*}{$1-1 \mathrm{abf}$} & \multirow{2}{*}{$1-1 a b$} & $\mathrm{M}$ & \multirow{2}{*}{$1-6$} & \multirow{4}{*}{$\mathrm{AB}$} & \multirow{4}{*}{$1-5$} & $f$ \\
\hline & & 10- & & & & $\mathrm{I}$ \\
\hline \multirow{2}{*}{$8-3 a b f$} & \multirow{2}{*}{$8-3 a b$} & $\mathrm{M}$ & \multirow{2}{*}{$1-6$} & & & $\mathrm{~m}$ \\
\hline & & $10-$ & & & & 111 \\
\hline \multirow{2}{*}{$21 \mathrm{tuf}$} & \multirow{2}{*}{$21 \mathrm{tu}$} & $\mathrm{M}$ & \multirow{2}{*}{16} & \multirow{4}{*}{$\mathrm{Tu}$} & \multirow{4}{*}{$1-5$} & $f$ \\
\hline & & 10 & & & & $\mathrm{I}$ \\
\hline \multirow{2}{*}{ 7-3tuf } & \multirow{2}{*}{$7-3$ tu } & $\mathrm{M}$ & \multirow{2}{*}{$1-6$} & & & $\mathrm{~m}$ \\
\hline & & $10-$ & & & & $\mathrm{m}$ \\
\hline \multirow{2}{*}{ 1-1wikf } & \multirow{2}{*}{$1-1$ wik } & $\mathrm{M}$ & \multirow{2}{*}{$1-6$} & \multirow{3}{*}{ WIK } & \multirow{3}{*}{$1-5$} & $\mathrm{f}$ \\
\hline & & $10-$ & & & & \\
\hline 1-2wikf & 1-2wik & $\frac{M}{10-}$ & $1-6$ & & & $\mathrm{~m}$ \\
\hline
\end{tabular}

Acute treatment (2) sequencing sample key breakdown. Maternal line: pair ID, strain, $f$ (female). Treated fish: pair ID, strain, [either] M (methanol control) or 10- (10ng EE2/L tx), 1-6 (individual ID). Baseline fish: strain, 1-5 (individual ID), [either] $\mathrm{f}$ (female) or $\mathrm{m}$ (male). 


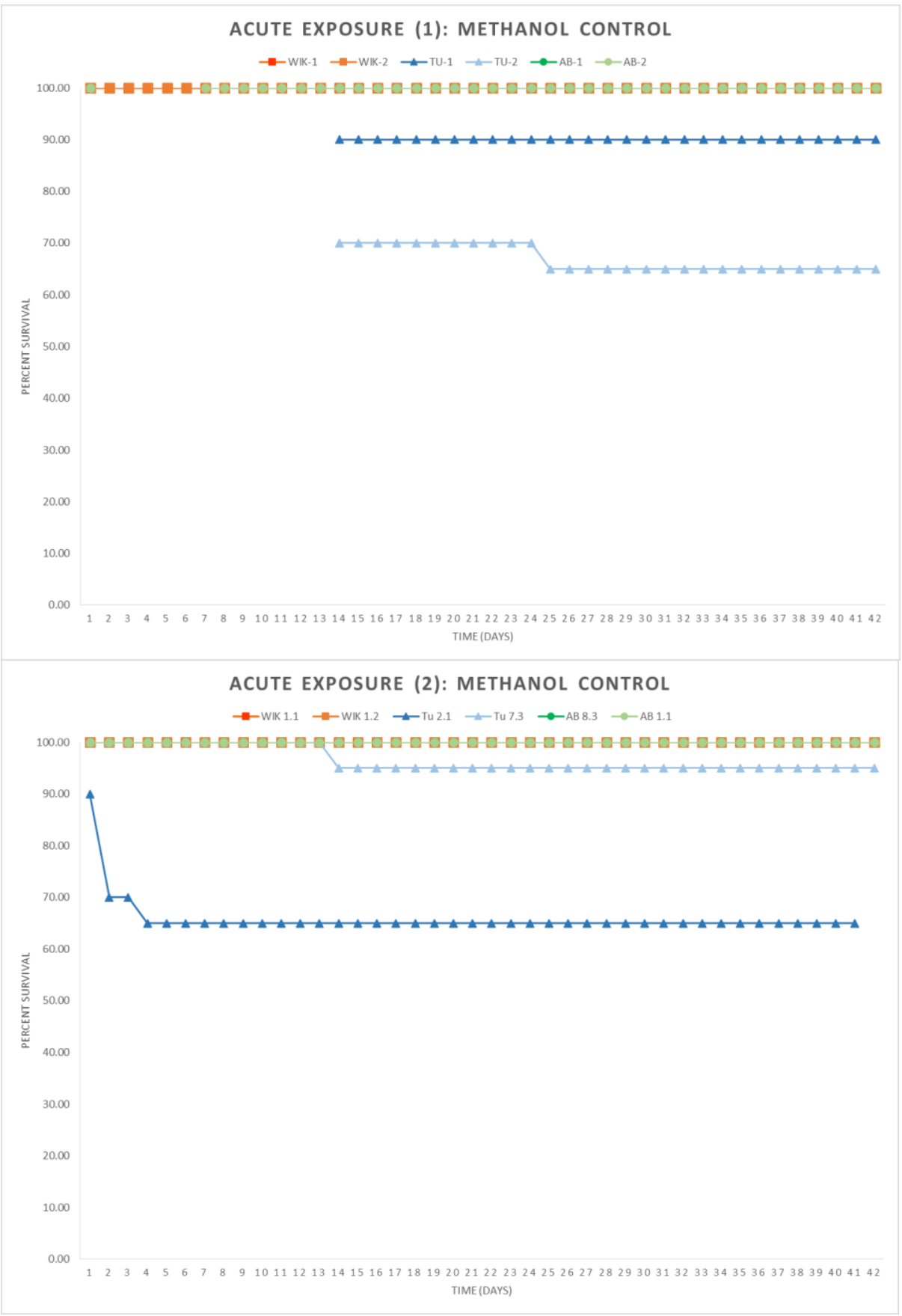

Figure 9: Percent survival against 42 day treatment duration. Two acute exposure series were performed (1) and (2) of control (a) group (with $\leq 0.00025 \%$ methanol, representing the greatest amount of methanol used as an EE2 solvent in highest treatment) of three zebrafish strains: AB (green), Tu (blue), and WIK (orange). Two maternal/paternal pairs were used per strain to generate full-sib offspring represented by two shades of associated color (ie dark blue for pair one and light blue for pair two of Tu). Maternal/paternal $(\mathrm{m} / \mathrm{p})$ lines used in acute exposure (1) were different than $\mathrm{m} / \mathrm{p}$ lines used in (2). 


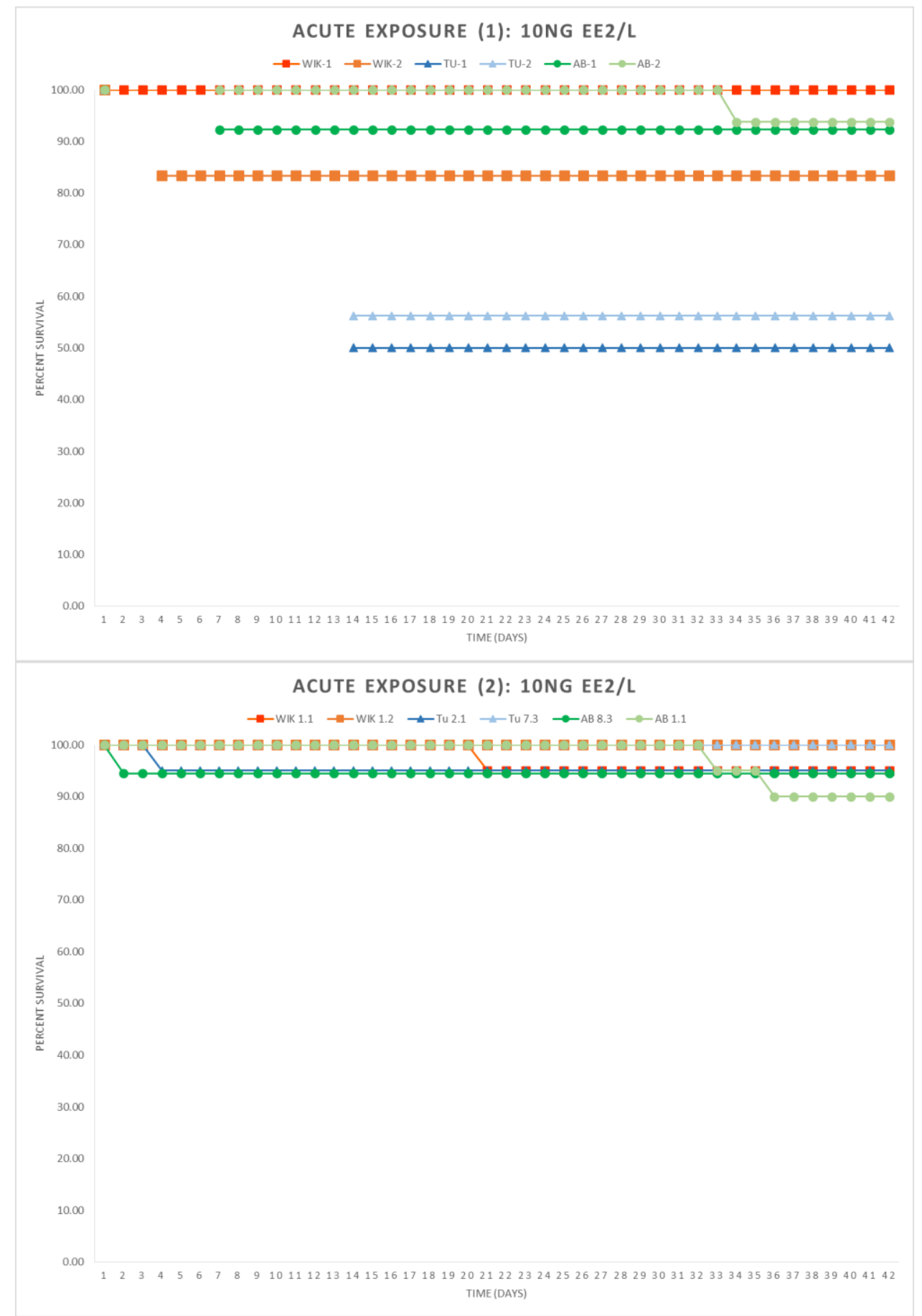

Figure 10: Percent survival against 42 day exposure (1) and (2) for 10ng EE2/L treatment groups of three zebrafish strains: AB (green), Tu (blue), and WIK (orange) from two pairs per strain. 


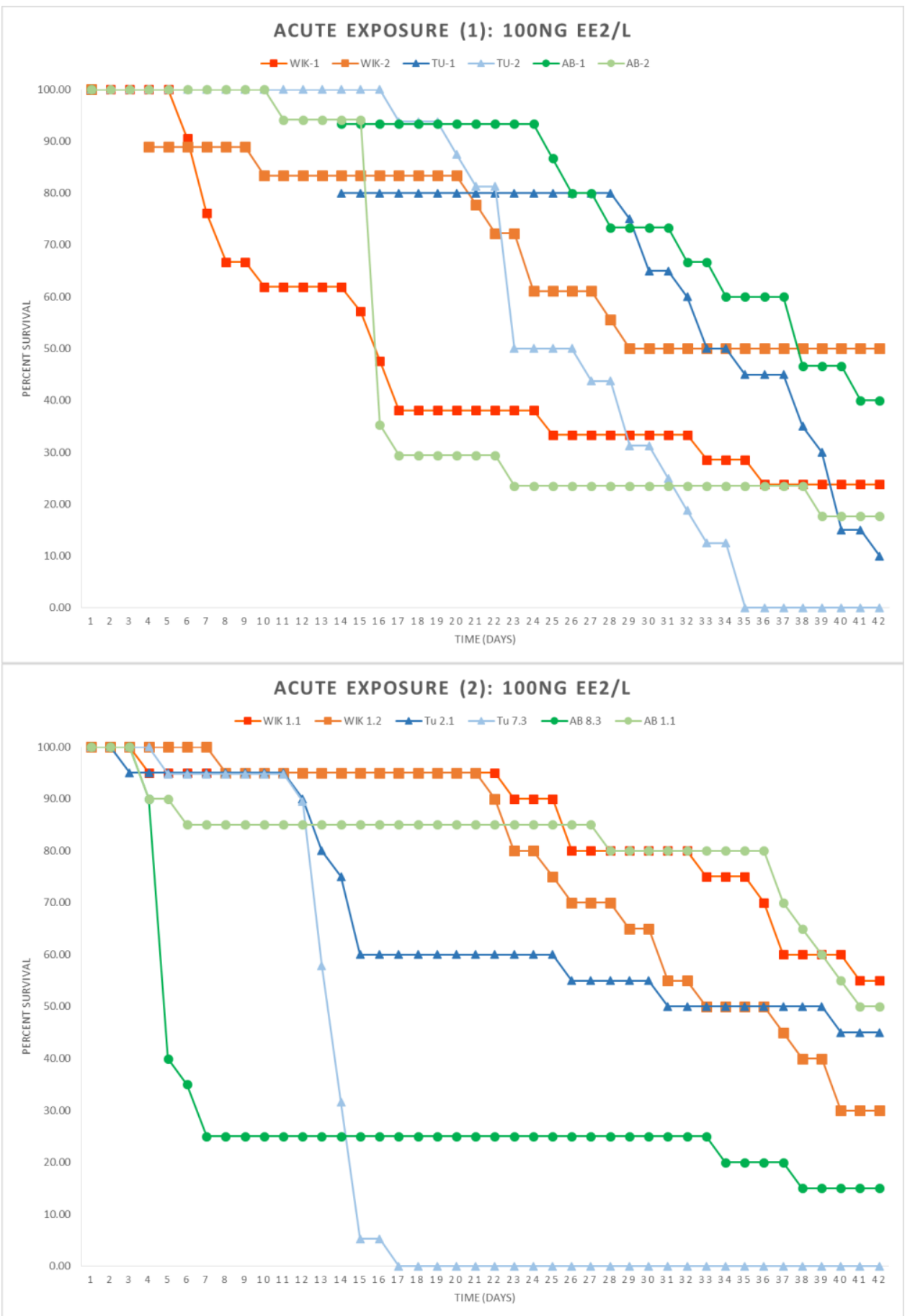

Figure 11: Percent survival against 42 day exposure (1) and (2) for 100ng EE2/L treatment groups of three zebrafish strains: AB (green), Tu (blue), and WIK (orange) from two pairs per strain. 

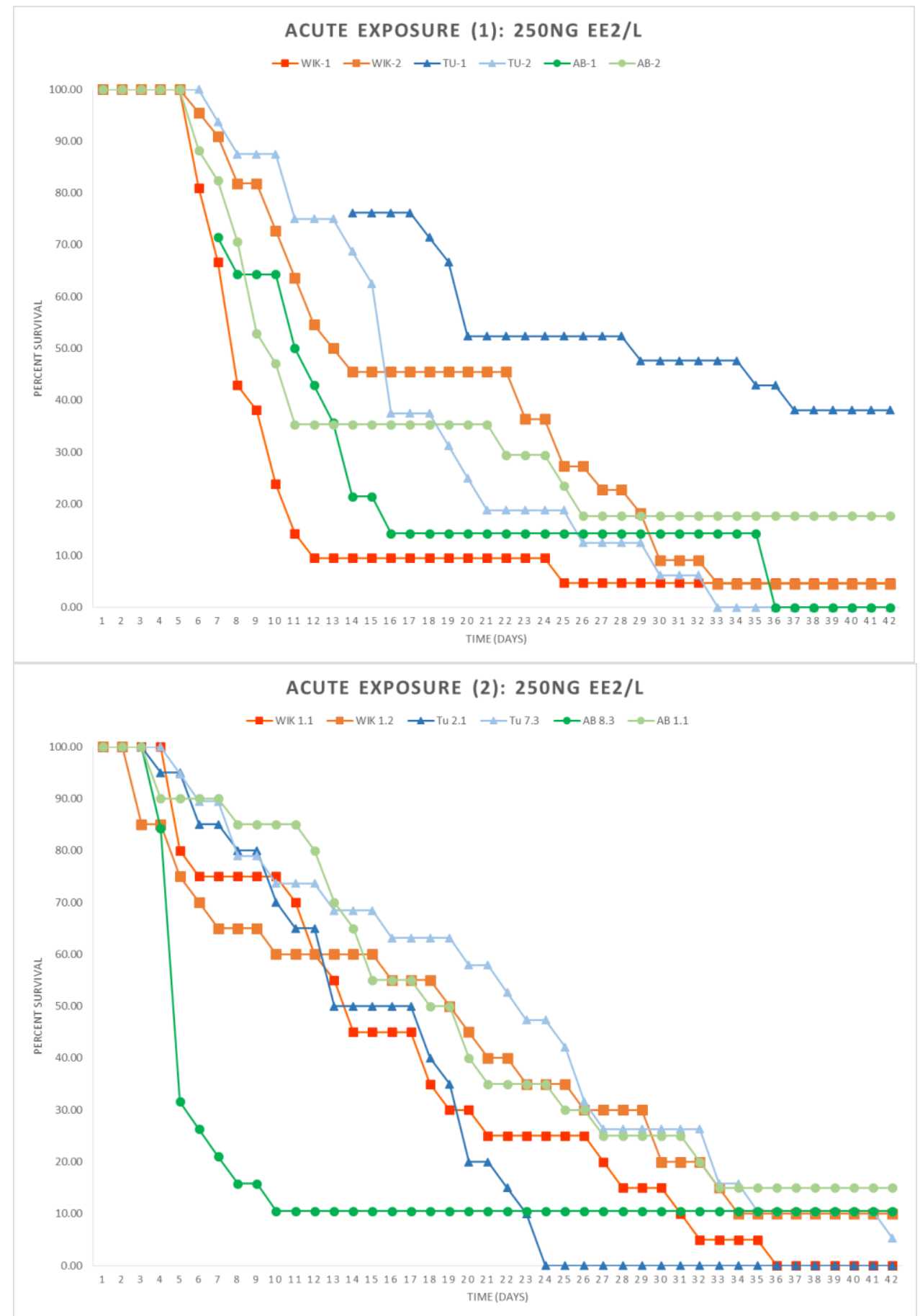

Figure 12: Percent survival against 42 day exposure (1) and (2) for 250ng EE2/L treatment groups of three zebrafish strains: AB (green), Tu (blue), and WIK (orange) from two pairs per strain. 


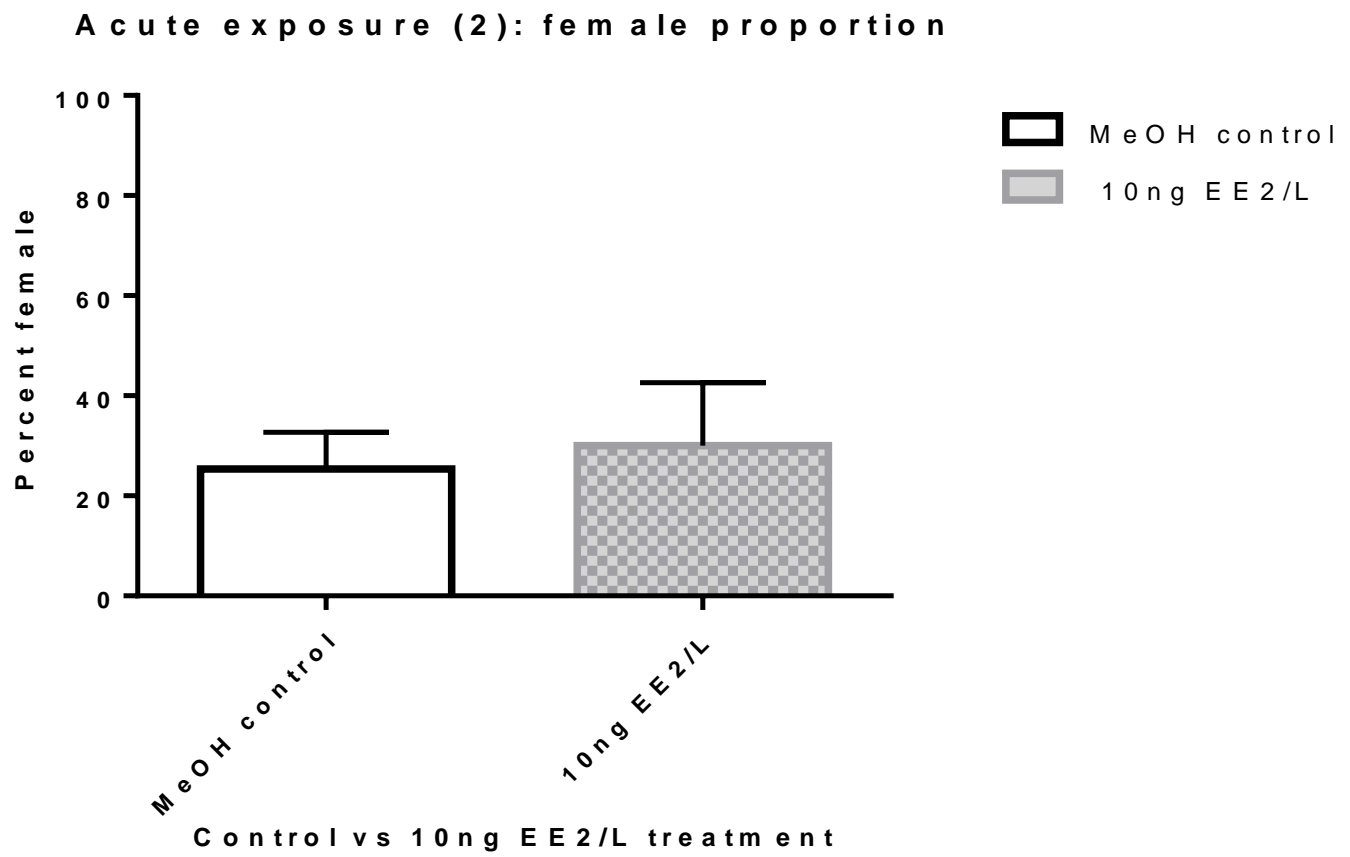

Figure 13: Proportion female between control and 10ng EE2/L treated fish after 42 day exposure $(\mathrm{p}<0.05)$.

\begin{tabular}{r|r|c|c|c}
\hline \multicolumn{5}{c}{ BETWEEN RUN PRECISION \& ACCURACY } \\
\hline ng/L & ng/L & & $\%$ & $\%$ differ \\
\hline nominal & mean calc & SD & RSD & accuracy \\
\hline 1.0 & 1.0 & 0.2 & $19.0 \%$ & $105 \%$ \\
\hline 2.5 & 2.0 & 0.3 & $14.3 \%$ & $81 \%$ \\
\hline 10.0 & 11.6 & 1.9 & $16.1 \%$ & $116 \%$ \\
\hline 50.0 & 49.3 & 8.2 & $16.5 \%$ & $99 \%$ \\
\hline 100.0 & 97.2 & 17.0 & $17.4 \%$ & $97 \%$ \\
\hline 250.0 & 248.7 & 22.9 & $9.2 \%$ & $99 \%$ \\
\hline WITHIN RUN PRECISION \& ACCURACY \\
\hline ng/L & ng/L & \multicolumn{4}{|c}{$\%$} & $\%$ differ \\
\hline nominal & mean calc & SD & RSD & accuracy \\
\hline 1.0 & 1.1 & 0.1 & $8.0 \%$ & $109 \%$ \\
\hline 2.5 & 2.3 & 0.3 & $11.8 \%$ & $92 \%$ \\
\hline 10.0 & 9.6 & 0.8 & $8.3 \%$ & $96 \%$ \\
\hline 50.0 & 47.3 & 2.5 & $5.2 \%$ & $95 \%$ \\
\hline 100.0 & 111.2 & 6.7 & $6.0 \%$ & $111 \%$ \\
\hline 250.0 & 242.0 & 18.0 & $7.4 \%$ & $97 \%$
\end{tabular}

Table 3: Between and within run precision and accuracy for acute exposure (2) calibrants and high-low QCs. 


\begin{tabular}{l|r|l|l|l} 
& mean & SD & RSD & \\
\hline $250 \mathrm{ng} / \mathrm{L}$ & 228.983 & 134.9086 & $58.9 \%$ & $\mathrm{n}=5$ \\
\hline $100 \mathrm{ng} / \mathrm{L}$ & 122.164 & 18.55928 & $15.2 \%$ & $\mathrm{n}=4$ \\
\hline $10 \mathrm{ng} / \mathrm{L}$ & 15.580 & 9.510547 & $61.0 \%$ & $\mathrm{n}=5$
\end{tabular}

Table 4: Calculated mean concentrations of EE2 in mixing tank samples from acute exposure (2).

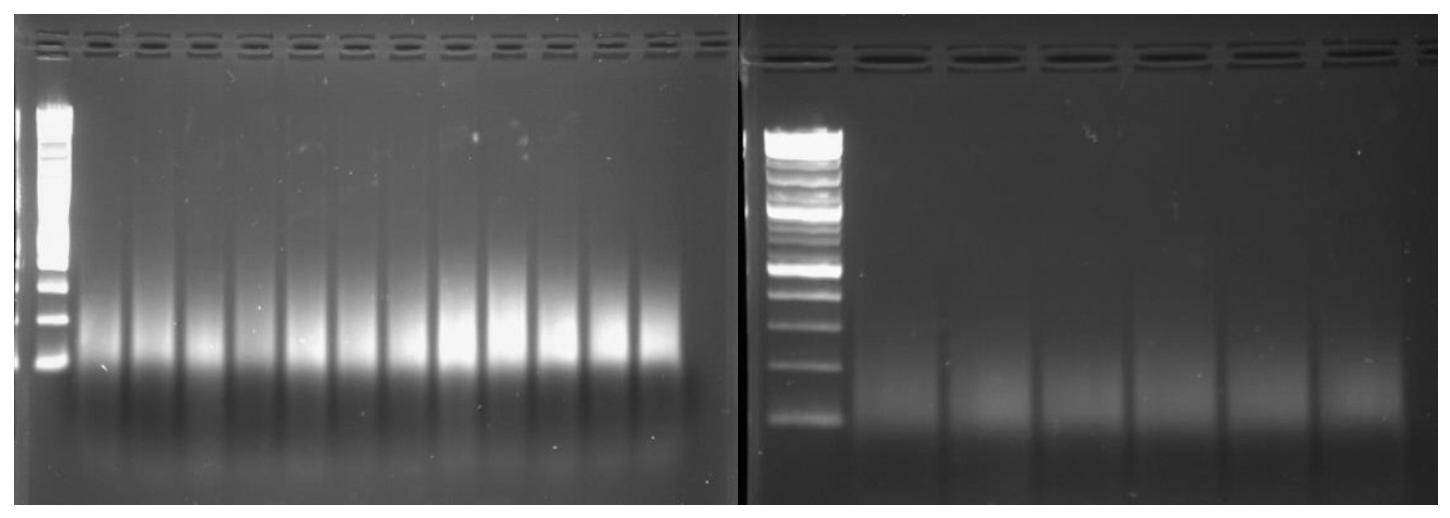

Figure 14: Gel verification examples showing samples post-sonication to ensure target fragment size of 150-200bp was achieved. Far left lane, 100bp ladder counting from the bottom up: 100, 200, 300, 400bp.

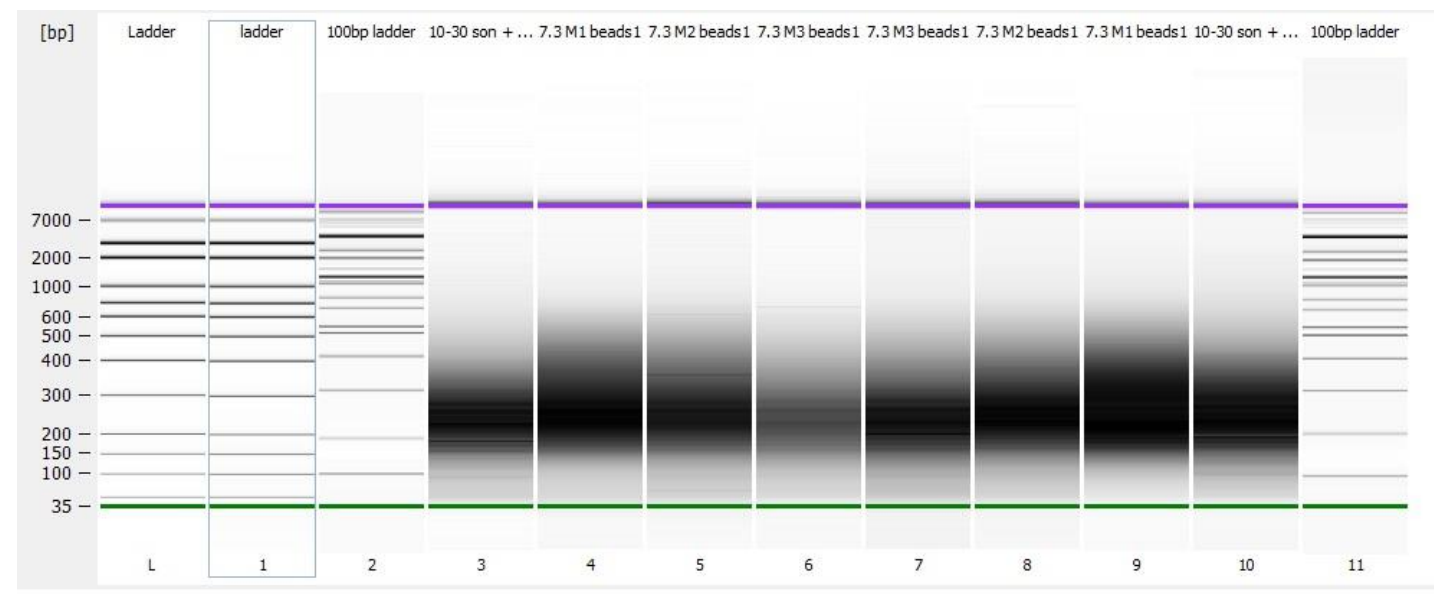

Figure 15: Bioanalyzer results during quality control check of post-sonication and post-AMPure bead clean-up 1 for library preparation of earliest samples (7-3tuM set) against 100bp ladder. 
Table 5: Average sequence coverage for acute exposure (2) per maternal sample

\begin{tabular}{c|c}
\hline Maternal ID & coverage \\
\hline 1-1abf & 82567 \\
\hline 8-3abf & 78815 \\
\hline 7-3tuf & 94984 \\
\hline 1-1wikf & 74776 \\
\hline 1-2wikf & 91816 \\
\hline
\end{tabular}

Table 6: Average sequence coverage for acute exposure (2) AB pairs

\begin{tabular}{c|c|c|c|c|c|c|c}
\hline $\begin{array}{c}\text { Sample } \\
\text { ID }\end{array}$ & coverage & $\begin{array}{c}\text { Sample } \\
\text { ID }\end{array}$ & coverage & $\begin{array}{c}\text { Sample } \\
\text { ID }\end{array}$ & coverage & $\begin{array}{c}\text { Sample } \\
\text { ID }\end{array}$ & coverage \\
\hline 1-1abM1 & 91037 & $\begin{array}{c}\mathbf{1 - 1 a b 1 0 -} \\
\mathbf{1}\end{array}$ & 94143 & $\mathbf{8 - 3 a b M 1}$ & 50489 & $\mathbf{8 - 3 a b 1 0 . 1}$ & 47506 \\
\hline 1-1abM2 & 93196 & $\begin{array}{c}\mathbf{1 - 1 a b 1 0 -} \\
\mathbf{2}\end{array}$ & 90290 & $\mathbf{8 - 3 a b M 2}$ & 90640 & $\mathbf{8 - 3 a b 1 0 . 2}$ & 55063 \\
\hline 1-1abM3 & 96062 & $\begin{array}{c}\mathbf{1 - 1 a b 1 0 -} \\
\mathbf{3}\end{array}$ & 91187 & $\mathbf{8 - 3 a b M 3}$ & 95426 & $\mathbf{8 - 3 a b 1 0 . 3}$ & 97797 \\
\hline 1-1abM4 & 83178 & $\begin{array}{c}\mathbf{1 - 1 a b 1 0 -} \\
\mathbf{4}\end{array}$ & 96033 & $\mathbf{8 - 3 a b M 4}$ & 92141 & $\mathbf{8 - 3 a b 1 0 . 4}$ & 93809 \\
\hline 1-1abM5 & 83372 & $\begin{array}{c}\mathbf{1 - 1 a b 1 0 -} \\
\mathbf{5}\end{array}$ & 94783 & $\mathbf{8 - 3 a b M 5}$ & 95692 & $\mathbf{8 - 3 a b 1 0 . 5}$ & 91471 \\
\hline 1-1abM6 & 94503 & $\begin{array}{c}\mathbf{1 - 1 a b 1 0 -} \\
\mathbf{6}\end{array}$ & 96294 & $\mathbf{8 - 3 a b M 6}$ & 94090 & $\mathbf{8 - 3 a b 1 0 . 6}$ & 95028 \\
\hline
\end{tabular}

Table 7: Average sequence coverage for acute exposure

(2) Tu pair

\begin{tabular}{c|c|c|c}
\hline Sample ID & coverage & Sample ID & coverage \\
\hline 7-3tuM1 & 92037 & $\mathbf{7 - 3 t u 1 0 - 1}$ & 94186 \\
\hline 7-3tuM2 & 90003 & $\mathbf{7 - 3 t u 1 0 - 2}$ & 94150 \\
\hline 7-3tuM3 & 94058 & $\mathbf{7 - 3 t u 1 0 - 3}$ & 93702 \\
\hline 7-3tuM4 & 88071 & $\mathbf{7 - 3 t u 1 0 - 4}$ & 74148 \\
\hline 7-3tuM5 & 92173 & $\mathbf{7 - 3 t u 1 0 - 5}$ & 88435 \\
\hline 7-3tuM6 & 90252 & $\mathbf{7 - 3 t u 1 0 - 6}$ & 94844
\end{tabular}


Table 8: Average sequence coverage for acute exposure (2) WIK pairs

\begin{tabular}{c|c|c|c|c|c|c|c}
\hline $\begin{array}{c}\text { Sample } \\
\text { ID }\end{array}$ & coverage & $\begin{array}{c}\text { Sample } \\
\text { ID }\end{array}$ & coverage & $\begin{array}{c}\text { Sample } \\
\text { ID }\end{array}$ & coverage & $\begin{array}{c}\text { Sample } \\
\text { ID }\end{array}$ & coverage \\
\hline $\begin{array}{c}\text { 1- } \\
\text { 1wikM1 }\end{array}$ & 93145 & $\begin{array}{c}\mathbf{1 -} \\
\text { 1wik10-1 }\end{array}$ & 94683 & $\begin{array}{c}\mathbf{1 -} \\
\text { 2wikM1 }\end{array}$ & 94996 & $\begin{array}{c}\text { 1- } \\
\mathbf{2 w i k 1 0 - 1}\end{array}$ & 95182 \\
\hline $\begin{array}{c}\text { 1- } \\
\text { 1wikM2 }\end{array}$ & 94030 & $\begin{array}{c}\mathbf{1 -} \\
\text { 1wik10-2 }\end{array}$ & 95744 & $\begin{array}{c}\mathbf{1 -} \\
\mathbf{2 w i k M 2}\end{array}$ & 93616 & $\begin{array}{c}\mathbf{1 -} \\
\mathbf{2 w i k 1 0 - 2}\end{array}$ & 93780 \\
\hline $\begin{array}{c}\mathbf{1 -} \\
\text { 1wikM3 }\end{array}$ & 95093 & $\begin{array}{c}\mathbf{1 -} \\
\mathbf{1 w i k 1 0 - 3}\end{array}$ & 95843 & $\begin{array}{c}\mathbf{1 -} \\
\mathbf{2 w i k M 3}\end{array}$ & 92525 & $\begin{array}{c}\mathbf{1 -} \\
\mathbf{2 w i k 1 0 - 3}\end{array}$ & 89616 \\
\hline $\begin{array}{c}\mathbf{1 -} \\
\text { 1wikM4 }\end{array}$ & 94538 & $\begin{array}{c}\mathbf{1 -} \\
\mathbf{1 w i k 1 0 - 4}\end{array}$ & 95749 & $\begin{array}{c}\mathbf{1 -} \\
\mathbf{2 w i k M 4}\end{array}$ & 94271 & $\begin{array}{c}\mathbf{1 -} \\
\mathbf{2 w i k 1 0 - 4}\end{array}$ & 95885 \\
\hline $\begin{array}{c}\mathbf{1 -} \\
\text { 1wikM5 }\end{array}$ & 85296 & $\begin{array}{c}\mathbf{1 -} \\
\mathbf{1 w i k 1 0 - 5}\end{array}$ & 95801 & $\begin{array}{c}\mathbf{1 -} \\
\mathbf{2 w i k M 5}\end{array}$ & 92849 & $\begin{array}{c}\mathbf{1 -} \\
\mathbf{2 w i k 1 0 - 5}\end{array}$ & 92796 \\
\hline $\begin{array}{c}\mathbf{1 -} \\
\text { 1wikM6 }\end{array}$ & 95226 & $\begin{array}{c}\mathbf{1 -} \\
\mathbf{1 w i k 1 0 - 6}\end{array}$ & 95454 & $\begin{array}{c}\mathbf{1 -} \\
\mathbf{2 w i k M 6}\end{array}$ & 96144 & $\begin{array}{c}\mathbf{1 -} \\
\mathbf{2 w i k 1 0 - 6}\end{array}$ & 95890
\end{tabular}

Table 9: Average sequence coverage for baseline fish

\begin{tabular}{c|c|c|c|c|c}
\hline Sample ID & coverage & Sample ID & coverage & Sample ID & coverage \\
\hline ab1f & 94627 & tu1f & 91613 & wik1f & 94509 \\
\hline ab2f & 76843 & tu2f & 89338 & wik2f & 94233 \\
\hline ab3f & 81699 & tu3f & 91462 & wik3f & 96490 \\
\hline ab4f & 91078 & tu4f & 90188 & wik4f & 92016 \\
\hline ab5f & 95675 & tu5f & 87682 & wik5f & 97850 \\
\hline ab1m & 91327 & tu1m & 80310 & wik1m & 79232 \\
\hline $\mathbf{a b 2 m}$ & 73759 & tu2m & 82806 & wik2m & 68031 \\
\hline $\mathbf{a b 4 m}$ & 84021 & tu3m & 89455 & wik3m & 79096 \\
\hline ab5m & 92975 & tu4m & 39080 & wik4m & 90175 \\
\hline & & & 91244 & wik5m & 93883
\end{tabular}



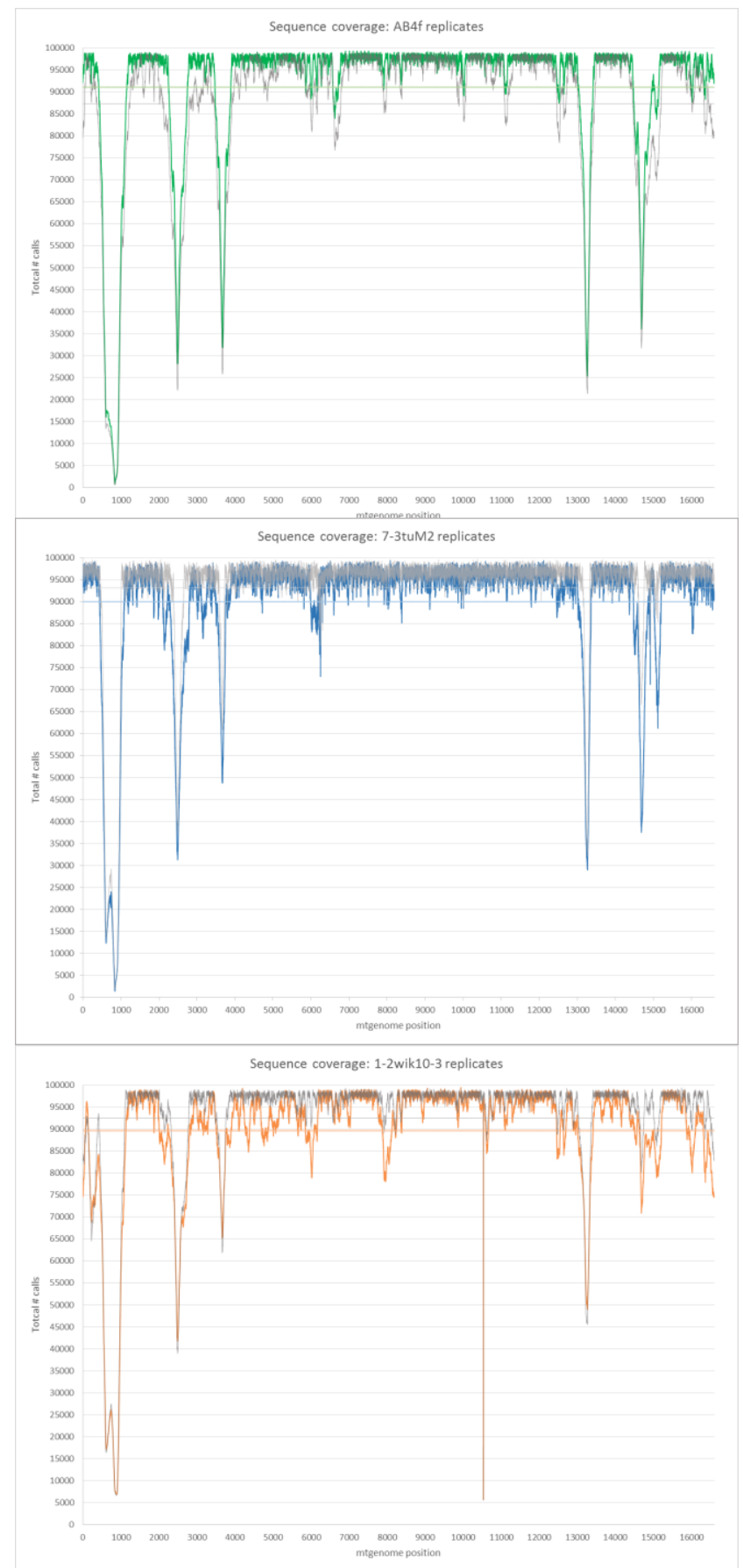

Figure 16: Replicates overlaid sequence coverage to demonstrate general trend of coverage across mtgenome. Each sample is associated strain color (AB-green, Tu-blue, WIK-orange) with grey for same sample replicate data. Linear lines represent average sequence coverage. 
Table 10: Alignment scores for $\mathrm{AB}$ acute exposure sequencing samples

\begin{tabular}{c|c|c|c|c|c}
\hline AB control ID & Total reads & $\begin{array}{c}\text { Percent } \\
\text { aligned to } \\
\text { mtgenome }\end{array}$ & $\begin{array}{c}\text { AB 10ng EE2/L } \\
\text { ID }\end{array}$ & Total reads & $\begin{array}{c}\text { Percent } \\
\text { aligned to } \\
\text { mtgenome }\end{array}$ \\
\hline $1-1$ abM1 & 9785469 & $97.15 \%$ & $1-1 \mathrm{ab} 10-1$ & 18649660 & $98.35 \%$ \\
\hline $1-1 \mathrm{abM} 2$ & 10307998 & $97.55 \%$ & $1-1 \mathrm{ab} 10-2$ & 10046209 & $92.18 \%$ \\
\hline $1-1 \mathrm{abM} 3$ & 14540774 & $97.30 \%$ & $1-1 \mathrm{ab} 10-3$ & 8392656 & $97.60 \%$ \\
\hline $1-1 \mathrm{abM} 4$ & 9227333 & $78.14 \%$ & $1-1 \mathrm{ab} 10-4$ & 12932520 & $98.31 \%$ \\
\hline $1-1 \mathrm{abM} 5$ & 7497260 & $96.41 \%$ & $1-1 \mathrm{ab} 10-5$ & 12384164 & $82.28 \%$ \\
\hline 1-1abM6 & 15135670 & $98.24 \%$ & $1-1 \mathrm{ab} 10-6$ & 13416254 & $95.35 \%$ \\
\hline 8-3abM1 & 10327609 & $96.64 \%$ & $8-3 \mathrm{ab} 10-2$ & 9041167 & $88.27 \%$ \\
\hline 8-3abM2 & 15593195 & $98.10 \%$ & $8-3 \mathrm{ab} 10-2$ & 10849477 & $77.96 \%$ \\
\hline 8-3abM3 & 12370702 & $98.55 \%$ & $8-3 \mathrm{ab} 10-3$ & 46985325 & $98.37 \%$ \\
\hline 8-3abM4 & 12545758 & $96.91 \%$ & $8-3 \mathrm{ab} 10-4$ & 48892407 & $96.54 \%$ \\
\hline 8-3abM5 & 12833063 & $96.59 \%$ & $8-3 \mathrm{ab} 10-5$ & 46088237 & $95.95 \%$ \\
\hline 8-3abM6 & 11075324 & $97.53 \%$ & $8-3 \mathrm{ab} 10-6$ & 25023956 & $94.49 \%$ \\
\hline
\end{tabular}

Sequence alignment overview including total reads and percent reads aligning to mtgenome per sample. 
Table 11: Alignment scores for Tu acute exposure sequencing samples

\begin{tabular}{c|c|c|c|c|c}
\hline Tu control ID & Total reads & $\begin{array}{c}\text { Percent } \\
\text { aligned to } \\
\text { mtgenome }\end{array}$ & $\begin{array}{c}\text { Tu 10ngEE2/L } \\
\text { ID }\end{array}$ & Total reads & $\begin{array}{c}\text { Percent } \\
\text { aligned to } \\
\text { mtgenome }\end{array}$ \\
\hline 7-3tuM1 & 9996541 & $96.22 \%$ & $7-3$ tu10-1 & 12202077 & $96.38 \%$ \\
\hline $7-3$ tuM2 & 9380990 & $95.24 \%$ & $7-3$ tu10-2 & 10783675 & $95.32 \%$ \\
\hline 7-3tuM3 & 12103602 & $97.89 \%$ & $7-3$ tu10-3 & 9709533 & $96.51 \%$ \\
\hline 7-3tuM4 & 8837643 & $88.90 \%$ & $7-3$ tu10-4 & 12448650 & $76.17 \%$ \\
\hline 7-3tuM5 & 9372289 & $96.82 \%$ & $7-3$ tu10-5 & 13161170 & $94.65 \%$ \\
\hline 7-3tuM6 & 9195572 & $97.59 \%$ & $7-3$ tu10-6 & 10863646 & $96.79 \%$ \\
\hline 7-3tuM2 - & 13484344 & $95.12 \%$ & $7-3 t u 10-1-R E P$ & 14157961 & $96.52 \%$ \\
\hline REP & & & & &
\end{tabular}

Sequence alignment overview including total reads and percent of reads aligning to mtgenome per sample. 
Table 12: Alignment scores for WIK acute exposure sequencing samples

\begin{tabular}{c|c|c|c|c|c}
\hline $\begin{array}{c}\text { WIK control } \\
\text { ID }\end{array}$ & Total reads & $\begin{array}{c}\text { Percent } \\
\text { aligned to } \\
\text { mtgenome }\end{array}$ & $\begin{array}{c}\text { WIK 10ngEE2/L } \\
\text { ID }\end{array}$ & Total reads & $\begin{array}{c}\text { Percent } \\
\text { aligned to } \\
\text { mtgenome }\end{array}$ \\
\hline 1-1wikM1 & 11483722 & $97.65 \%$ & $1-1$ wik10-1 & 17343530 & $94.73 \%$ \\
\hline $1-1 w i k M 2$ & 13647048 & $95.32 \%$ & $1-1$ wik10-2 & 22664699 & $97.95 \%$ \\
\hline $1-1 w i k M 3$ & 13007021 & $97.52 \%$ & $1-1$ wik10-3 & 15159812 & $98.46 \%$ \\
\hline $1-1 w i k M 4$ & 12026852 & $87.34 \%$ & $1-1$ wik10-4 & 13131655 & $96.35 \%$ \\
\hline $1-1 w i k M 5$ & 8499963 & $96.97 \%$ & $1-1$ wik10-5 & 12758576 & $91.01 \%$ \\
\hline $1-1 w i k M 6$ & 11600664 & $95.40 \%$ & $1-1$ wik10-6 & 15418596 & $97.06 \%$ \\
\hline $1-2 w i k M 1$ & 12591621 & $96.75 \%$ & $1-2 w i k 10-1$ & 13475882 & $97.80 \%$ \\
\hline $1-2 w i k M 2$ & 13081604 & $96.62 \%$ & $1-2 w i k 10-2$ & 13582151 & $92.57 \%$ \\
\hline $1-2 w i k M 3$ & 8791778 & $97.74 \%$ & $1-2 w i k 10-3$ & 9741137 & $91.16 \%$ \\
\hline $1-2 w i k M 4$ & 10431192 & $98.10 \%$ & $1-2 w i k 10-4$ & 11695032 & $97.43 \%$ \\
\hline $1-2 w i k M 5$ & 9229908 & $96.36 \%$ & $1-2 w i k 10-5$ & 10221356 & $97.70 \%$ \\
\hline $1-2 w i k M 6$ & 13149401 & $97.70 \%$ & $1-2 w i k 10-6$ & 13164686 & $97.80 \%$ \\
\hline & & & $1-2 w i k 10-3-R E P$ & 8390810 & $96.71 \%$ \\
\hline
\end{tabular}

Sequence alignment overview including total reads and percent of reads aligning to mtgenome per sample. 
Table 13: Alignment scores for baseline sequencing samples

\begin{tabular}{|c|c|c|c|c|c|c|c|c|}
\hline AB ID & $\begin{array}{l}\text { Total } \\
\text { reads }\end{array}$ & $\begin{array}{l}\text { Percent } \\
\text { aligned } \\
\text { to } \mathrm{mt} \\
\text { genome }\end{array}$ & $\begin{array}{l}\mathrm{Tu} \\
\mathrm{ID}\end{array}$ & $\begin{array}{l}\text { Total } \\
\text { reads }\end{array}$ & $\begin{array}{l}\text { Percent } \\
\text { aligned to } \\
\text { mtgenome }\end{array}$ & $\begin{array}{l}\text { WIK } \\
\text { ID }\end{array}$ & $\begin{array}{l}\text { Total } \\
\text { reads }\end{array}$ & $\begin{array}{c}\text { Percent } \\
\text { aligned } \\
\text { to } \mathrm{mt} \\
\text { genome }\end{array}$ \\
\hline ab1f & 11997623 & 96.33 & tu1f & 9770625 & 98.05 & wik1f & 17152404 & 97.46 \\
\hline$a b 2 f$ & 6748448 & 97.09 & tu2f & 10730290 & 97.96 & wik2f & 12065535 & 97.10 \\
\hline$a b 3 f$ & 8431181 & 98.28 & tu3f & 10937490 & 98.52 & wik3f & 25447115 & 98.85 \\
\hline$a b 4 f$ & 9358941 & 93.32 & tu4f & 10048129 & 97.68 & wik4f & 9592859 & 96.45 \\
\hline$a b 5 f$ & 48378428 & 98.58 & tu5f & 9698742 & 96.10 & wik5f & 75301641 & 98.49 \\
\hline ab1m & 9919322 & 97.73 & tu1m & 8152350 & 98.02 & wik1m & 8304676 & 94.49 \\
\hline $\mathrm{ab} 2 \mathrm{~m}$ & 7485225 & 96.14 & tu $2 \mathrm{~m}$ & 7482459 & 96.16 & wik2m & 6844795 & 94.96 \\
\hline $\mathrm{ab3m}$ & 8713971 & 94.45 & tu3m & 11928427 & 97.86 & wik3m & 9489164 & 97.16 \\
\hline $\mathrm{ab} 4 \mathrm{~m}$ & 10804879 & 70.17 & tu4m & 30001454 & 97.96 & wik4m & 12682535 & 97.71 \\
\hline ab5m & 14160408 & 97.09 & tu5m & 11999388 & 97.16 & wik5m & 14932480 & 97.24 \\
\hline $\begin{array}{c}\text { ab4m- } \\
\text { REP }\end{array}$ & 7444162 & 96.18 & & & & & & \\
\hline $\begin{array}{l}\text { ab4f- } \\
\text { REP }\end{array}$ & 7936482 & 98.00 & & & & & & \\
\hline
\end{tabular}

Sequence alignment overview including total reads and percent of reads aligning to mtgenome per sample. 
Table 14: Alignment scores for maternal sequencing samples

\begin{tabular}{c|c|c}
\hline Maternal ID & Total reads & $\begin{array}{c}\text { Percent aligned to } \\
\text { mtgenome }\end{array}$ \\
\hline $1-1 \mathrm{abf}$ & 8049851 & $94.12 \%$ \\
\hline $8-3 \mathrm{abf}$ & 7722668 & $93.41 \%$ \\
\hline z-1tuf & $\mathrm{n} / \mathrm{a}$ & $\mathrm{n} / \mathrm{a}$ \\
\hline 7-3tuf & 10166509 & $97.65 \%$ \\
\hline 1-1wikf & & $97.03 \%$
\end{tabular}

Sequence coverage overview including total reads and percent of reads aligning to mtgenome per sample. 

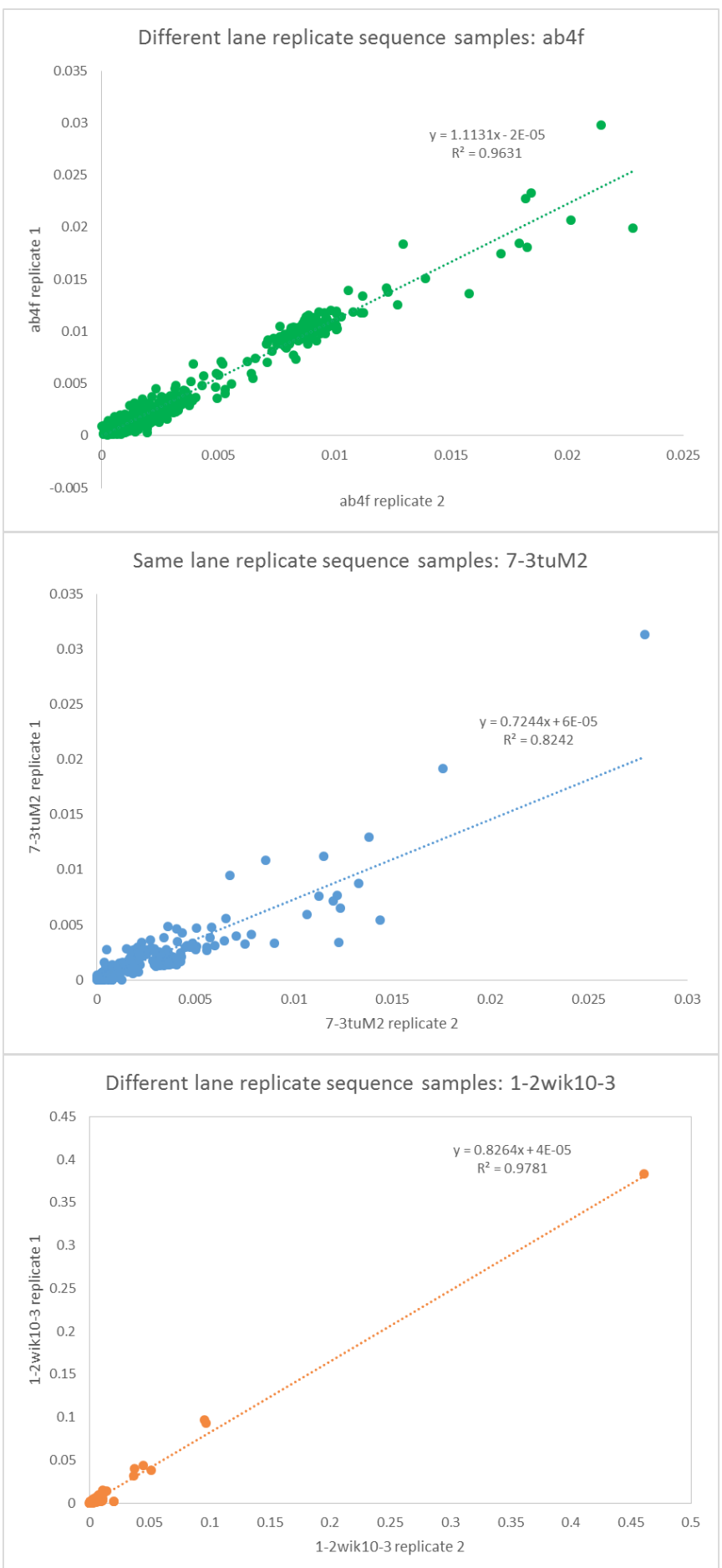

Figure 17: Linear regression of sequencing replicate heteroplasmy representing each strain $(\mathrm{AB}, \mathrm{Tu}$, and WIK), same vs different sequencing lanes between replicates, and differing groups (baseline, treated, and control). 
Table 15: Comparison of replicate samples sequencing design and values

\begin{tabular}{c|c|c|c|c}
\hline \multirow{2}{*}{ Lane design } & Group & $\begin{array}{c}\text { Replicate Sample } \\
\text { ID }\end{array}$ & Average error & Greatest error \\
\hline \multirow{2}{*}{ Same lane } & Control & 7-3tuM2 & 0.000069 & 0.0096 \\
\cline { 2 - 5 } & Treated & 7-3tu10-1 & 0.000104 & 0.029 \\
\hline \multirow{3}{*}{ Different lane } & Treated & 1-2wik10-3 & 0.000147 & 0.076 \\
\cline { 2 - 5 } & Baseline & ab4f & 0.000102 & 0.00037 \\
\cline { 2 - 5 } & Baseline & ab4m & 0.000121 & 0.0079
\end{tabular}

Table 16: Top ten sites on mtgenome with greatest heteroplasmic variance between replicates

\begin{tabular}{c|c|c|c|c|c}
\hline Site & $\mathbf{7 - 3 t u M 2}$ & $\mathbf{7 - 3 t u 1 0 - 1}$ & $\mathbf{1 - 2 w i k 1 0 - 3}$ & ab4f & ab4m \\
\hline $\mathbf{1}$ & 850 & 824 & 10535 & 834 & 834 \\
\hline $\mathbf{2}$ & 848 & 828 & 878 & 841 & 878 \\
\hline $\mathbf{3}$ & 834 & 825 & 870 & 843 & 804 \\
\hline $\mathbf{4}$ & 870 & 827 & 834 & 836 & 870 \\
\hline $\mathbf{5}$ & 827 & 837 & 13274 & 878 & 813 \\
\hline $\mathbf{6}$ & 825 & 833 & 6265 & 844 & 13202 \\
\hline $\mathbf{7}$ & 828 & 839 & 6176 & 908 & 855 \\
\hline $\mathbf{8}$ & 878 & 834 & 11293 & 825 & 4399 \\
\hline $\mathbf{9}$ & 824 & 846 & 11134 & 804 & 896 \\
\hline $\mathbf{1 0}$ & 839 & 843 & 5676 & 828 & 843 \\
\hline
\end{tabular}

Top ten sites on mtgenome with greatest rate of heteroplasmic variance between replicate samples. Sites highlighted in grey represent coding regions on the mtgenome. 


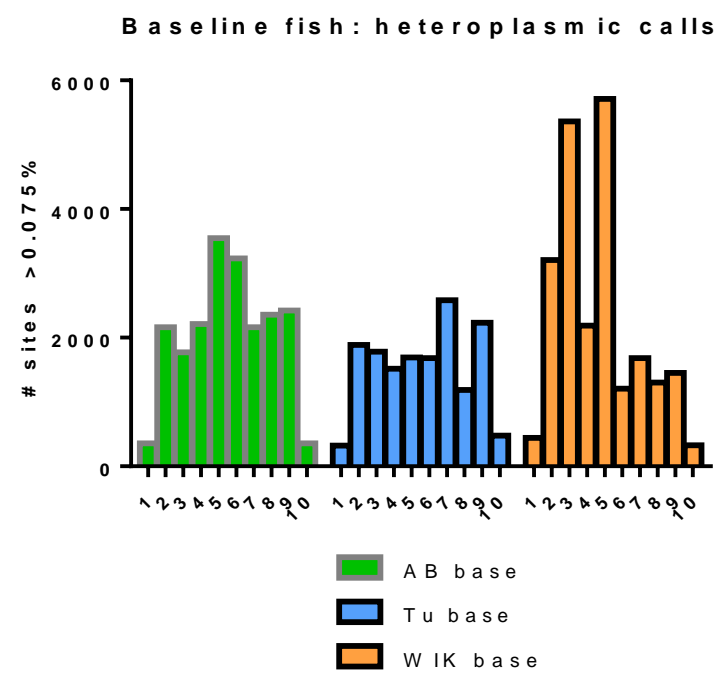

Figure 18: Breakdown of heteroplasmic sites $\geq 0.075 \%$ per individual of baseline fish, strains $A B$ (green), Tu (blue) and WIK (orange).
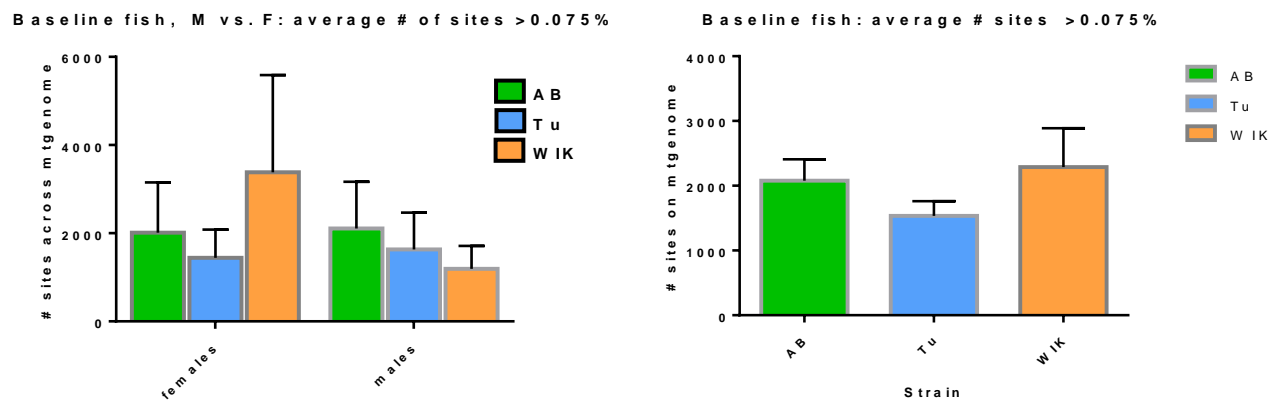

Figure 19: Breakdown of heteroplasmic sites $\geq 0.075 \%$ in baseline fish, strains $\mathrm{AB}$ (green), Tu (blue) and WIK (orange) either grouped by sex (left chart) or pooled sex (right chart). 
$8-3$ a b: heteroplas m ic calls
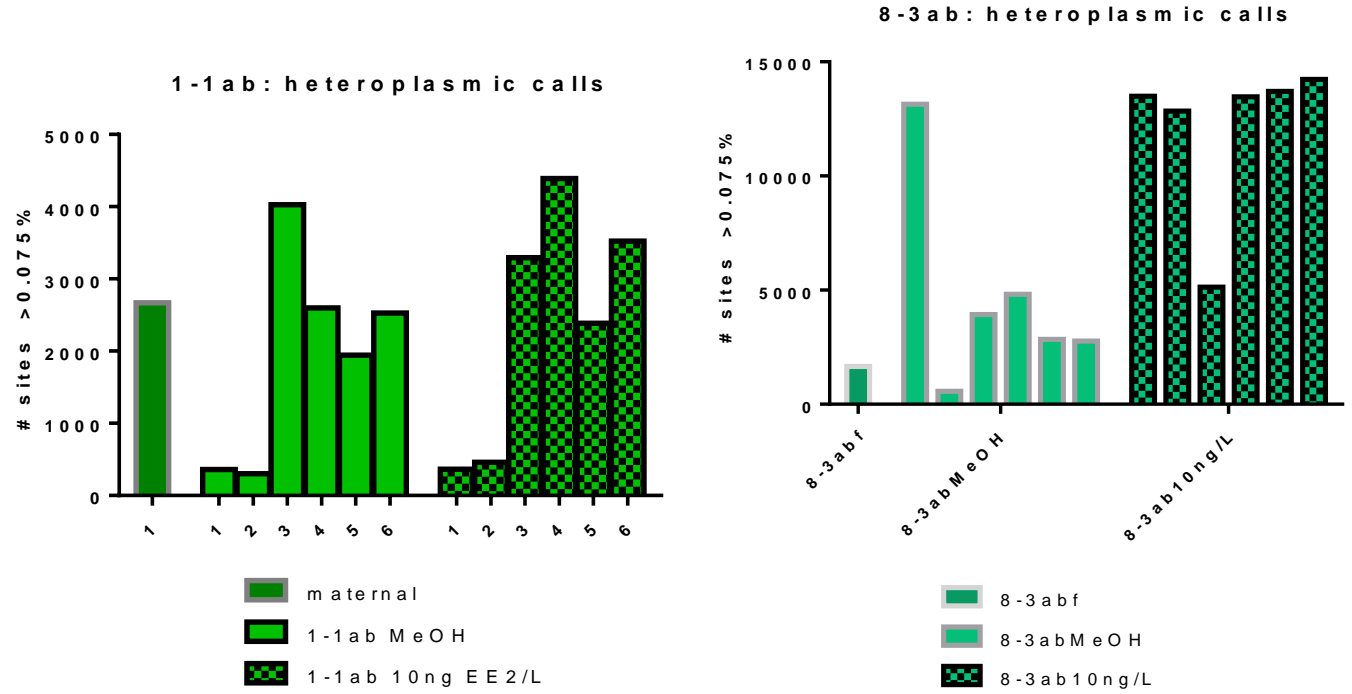

Figure 20: Breakdown of heteroplasmic sites $\geq 0.075 \%$ in maternal, $\mathrm{MeOH}$ control fish and 10ng EE2/L treated fish of two different $\mathrm{AB}$ pairs (1-1 left and 8-3 right).

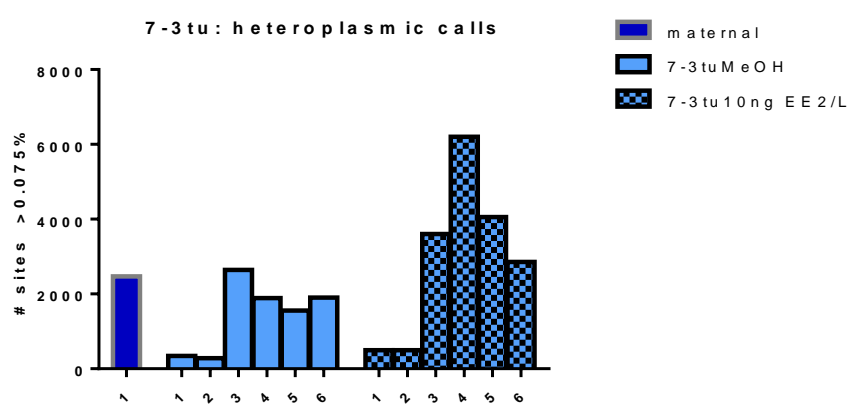

Figure 21: Breakdown of heteroplasmic sites $\geq 0.075 \%$ in maternal, $\mathrm{MeOH}$ control fish and $10 \mathrm{ng}$ EE2/L treated fish of Tu pair 7-3. 
1-1 wik: heteroplas m ic calls

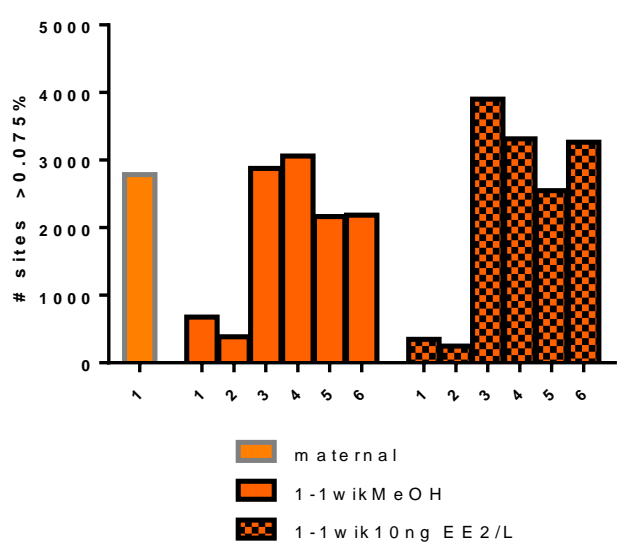

1-2 wik: heteroplas m ic calls

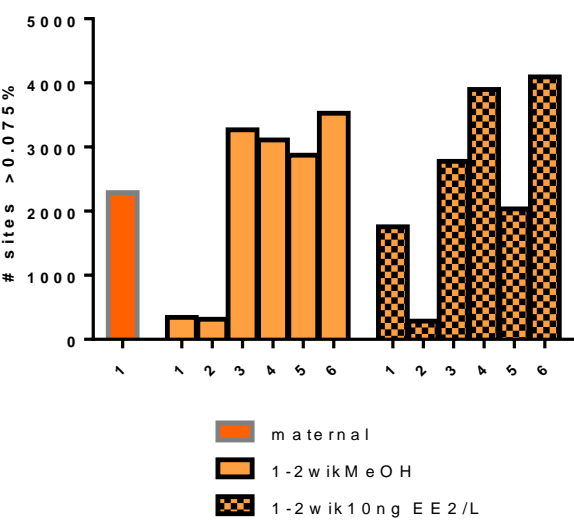

Figure 22: Breakdown of heteroplasmic sites $\geq 0.075 \%$ in maternal, MeOH control fish and 10ng EE2/L treated fish of two different WIK pairs (1-1 left and 1-2 right).

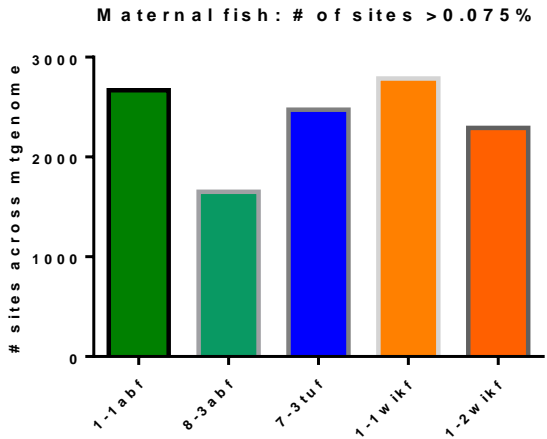

Figure 23: Breakdown of heteroplasmic sites $\geq 0.075 \%$ in maternal fish by pair and strain (AB pairs in shades of green, Tu in blue, and WIK pairs in shades of orange). 


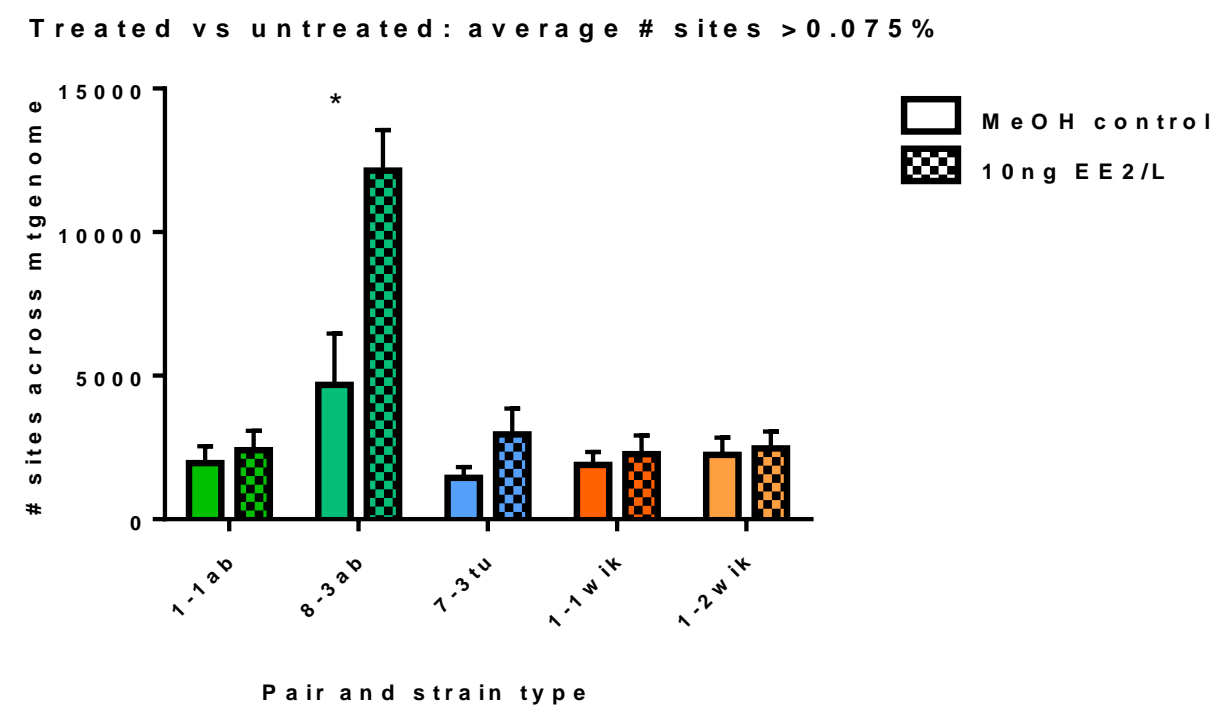

Figure 24: Breakdown of heteroplasmic sites $\geq 0.075 \%$ in control (no pattern) vs EE2 treated fish (block patterned) pair and strain (AB pairs in shades of green, Tu in blue, and WIK pairs in shades of orange). 
Treated vs untreated: average heteroplasm ic sites $>0.075 \%$

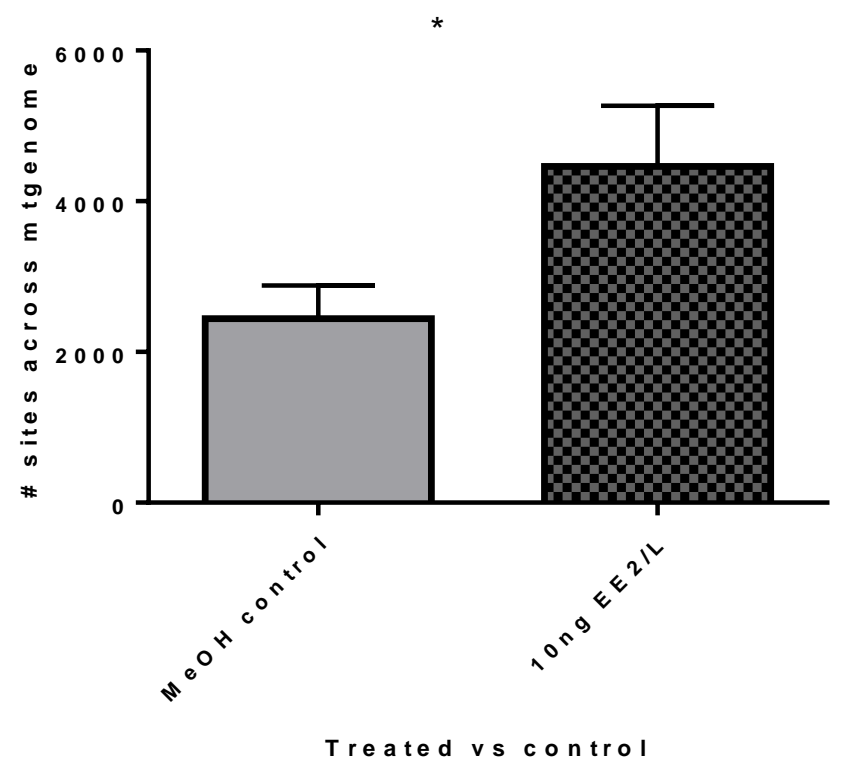

Figure 25: Breakdown of heteroplasmic sites $\geq 0.075 \%$ in $\mathrm{MeOH}$ control vs 10 ng EE2/L treated fish with strains pooled $(\mathrm{p}=0.03)$. 


\section{A II groups: average \# sites $>0.075 \%$}

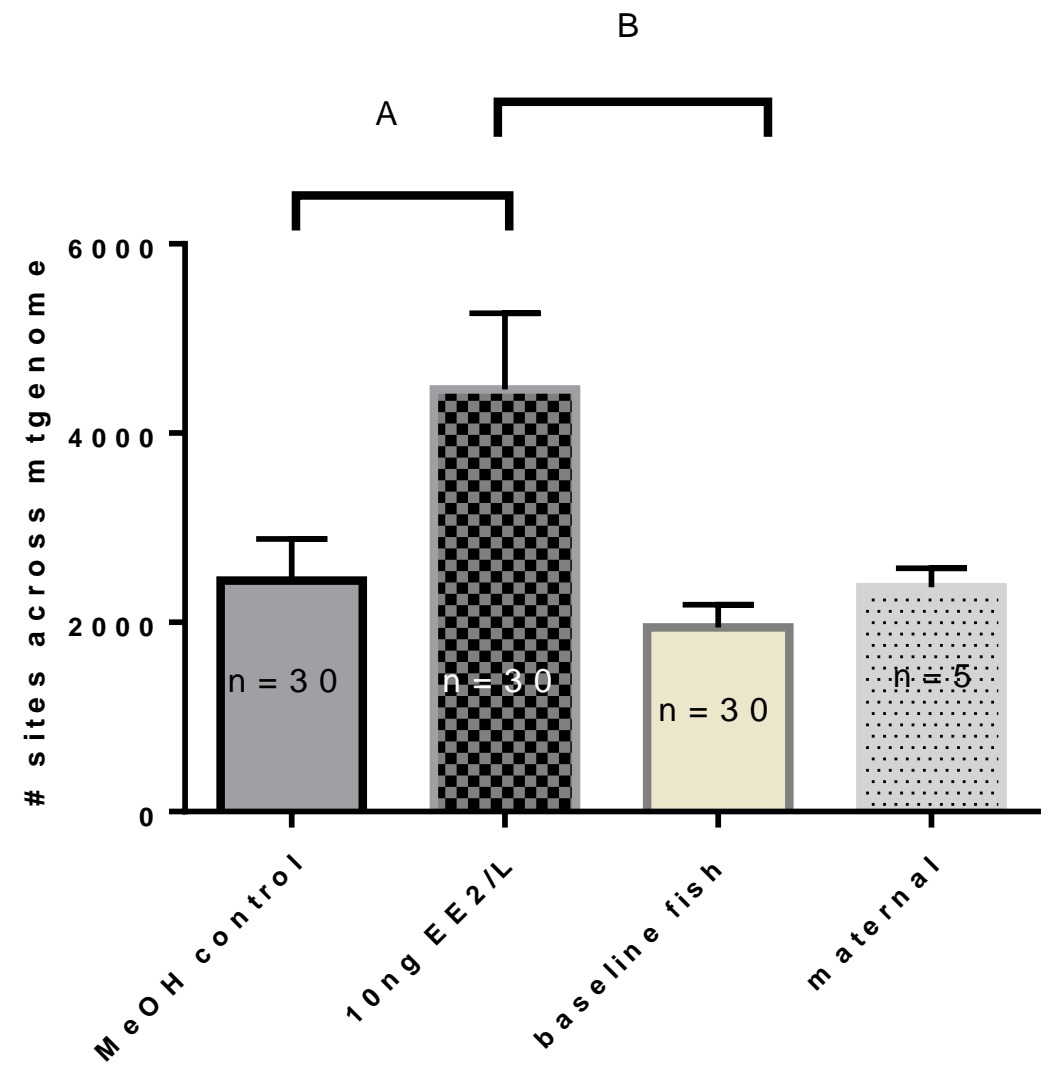

Figure 26: Breakdown of heteroplasmic sites $\geq 0.075 \%$ in each pooled group: $\mathrm{MeOH}$ control, $10 \mathrm{ng}$ EE2/L treated, baseline males and females, and maternal lines with $95 \% \mathrm{CI}$. 


\section{Chapter IV: Chronic treatment study \\ Introduction}

Environmental estrogens are a potent class of toxicants found ubiquitously in our environment as a result of their wide range of uses in commercial and industrial products including pesticides, plastics, and pharmaceuticals (Fenske et al., 2005; Ropero et al., 2006). Environmental estrogens present a significant health concern as numerous studies have demonstrated synthetic estrogens modulate and/or disrupt a variety of animal systems (Hecker \& Hollert, 2011). Such studies demonstrating physiological and developmental dysfunction on both aquatic and terrestrial vertebrate species within environmentally relevant concentrations include: reduced sperm production, altered spermantogenesis, intersex and complete feminization of males, altered female oogenesis and induction of aneuploidy in both sexes (Brown et al., 2008; Hunt et al., 2003; Kidd et al., 2007; Schultz et al., 2003; Takahashi \& Oishi, 2006; Tyler \& Jobling, 2008).

Despite it being widely accepted environmental estrogen exposure has the ability to interfere with a variety of vertebrate organ system, the extent of these effects are continually being investigated. Of these, the investigation of mitochondria as targets of toxicant exposure is of great concern as these organelles are responsible for cellular lifedeath decisions and also play critical roles in many other physiological processes (Cheng \& Ristow, 2013). With specific concern for mtgenome susceptibility from environmental estrogen exposure, we aimed to continue our investigation on the effects of $17 \alpha$ ethynylestradiol (EE2) exposure on mitochondrial genome stability. 
The objective of this study was to further investigate the effects of chronic exposure to the common environmental estrogen EE2 on mitochondrial DNA (mtDNA) stability in zebrafish from embryonic development through reproductive maturity. The current challenge of sequencing studies is often dependent on project funding. Unfortunately, financial constraints limited this study from being carried through completion of DNA sequencing and analysis; however, concerning effects of the test compound on common fish development, life cycle and sex determination were observed.

\section{Methods}

All fish were maintained in accordance to guidelines established by the Institutional Animal Care and Use Committee (IACUC) of Portland State University. Adult male and female zebrafish for generation of embryos used in chronic exposure, of the three common laboratory strains $\mathrm{AB}, \mathrm{Tu}$, and $\mathrm{WIK}$, less than one year of age were obtained from the Zebrafish International Resource Center (ZIRC). Fish were housed in 2.8-liter baffled flow-through tanks at a ratio of 1 male: 1 female with a stocking density of 10 or less fish per 2.8L tank in the Brown Zebrafish Aquatics Facility at Portland State University.

Fish were maintained on the Aquaneering Modular System with 4-stage central filtration providing continuous flow-through of recirculating water with a twice daily automated $10 \%$ water change. This system monitors parameters to stay within a narrow range. Aquatic facility had a photo period of $15 \mathrm{~h}$ light: $9 \mathrm{hr}$ dark. Fish were fed two times a day ad libitum with manufactured larvae power and live Artemia salina (brine shrimp). 
Sexually mature zebrafish $\sim 1$ year of age were set up as breeding pairs (1 male: 1 female) in Aquaneering Crossing Tanks with dividers to separate each pair. The following morning, dividers were removed and zebrafish were allowed to spawn for up to 5 hours. Spawning occurred within the aquatic facility using system water.

The test compound $17 \alpha$-ethynylestradiol (EE2) was obtained from Sigma-Aldrich ( $\geq 98 \%$ grade). EE2 was solubilized in $100 \%$ methanol at a ratio of $1 \mathrm{mg} / 1 \mathrm{~mL}$. A final working concentration of $1 \mathrm{ng} \mathrm{EE} 2 / \mu \mathrm{L}$ in $10 \%$ methanol was prepared as the final exposure working stock for spiking treatment water. Control fish were exposed to methanol at the highest EE2 treatment concentration, $5 \mu \mathrm{L} 100 \%$ methanol/liter tank water or $\leq 0.0005 \%$.

1-L amber glass bottles were filled with embryo media and spiked with EE2 working stock at respective treatment concentration 24-hours prior treatment start. Embryos were collected within 6 hours of morning divider removal and rinsed with untreated embryo media (Cold Harbor Springs 60x recipe). At a stocking density of $\leq 50$ embryos per petri dish, embryos were submersed in pre-mixed treatment water from one of five treatment groups: control, 1, 10, 25 or 50ng EE2/L. Petri dishes were placed in an incubator at $28^{\circ} \mathrm{C}$ for 5 days. Non-viable embryos were pulled and counts were recorded daily along with 2 water changes within the first five days post fertilization (dpf).

At $5 \mathrm{dpf}$, hatched larvae were transferred to $600 \mathrm{~mL}$ glass beakers in pre-mixed respective exposure water and started on concentrated rotifers twice daily ad libitum. Full sib, same strain, grouped larvae were housed in $600 \mathrm{~mL}$ beakers until 6 weeks of age with water changes every other day of pre-mixed exposure water. At 9dpf larvae were 
transitioned to live brine shrimp in addition to rotifers. Larval powder was introduced at 14 dpf. Fish were transitioned to powder only at 3 weeks and raised under these conditions until 6 weeks of age.

The exposure system used was as described in chapter III methods, acute exposure design. At six weeks of age, 20 full-sibs of each pair/strain/treatment were transferred to exposure system 1 gallon fish bowls. Common 20-gallon mixing tanks for respective treatments were filled and spiked with EE2 24-hours prior to transfer. Exposure to all fish at 50ng EE2/L was terminated after 6 weeks due abnormal growth patterns and impaired development. The remaining treatment groups: control, 1, 10, and 25ng EE2/L were continued until 6 months of age.

Fish were housed on the exposure system until 6 months of age. 30-second flushes refreshed water every other day. Waste debris was removed via turkey baster. Fish were maintained on a standard adult feeding schedule using fish powder ad libitum, twice daily.

At the 6 week transfer only 20 fish per strain/pair/treatment were continued through the 6 month exposure. Remaining fish were raised in $600 \mathrm{~mL}$ beaker conditions until 8 weeks of age for histological examination of gonadal tissue. At 8 weeks of age, juvenile larvae were euthanized in MS-222 (Sigma-Aldrich) at 300mg/L water at $\mathrm{pH} 8$ for $\geq 15$ minutes after fasting for 24 hours. Fish were transferred to $10 \%$ neutral buffered formalin (Sigma-Aldridge) for $\geq 24$ hours for fixation. Fixed fish were washed in $70 \%$ ethanol and transferred to embedding cassettes stored in $70 \%$ ethanol for further processing. Samples were sent to the Oregon Health and Science University, 
Histopathology Shared Resource Center where fish were paraffin embedded, sectioned onto slides using mid-sagittal cuts and H\&E stained.

After 6 months of exposure, fish were euthanized using MS-222 at a concentration of $300 \mathrm{mg} / \mathrm{L}$ water at $\mathrm{pH} 8$ for $\geq 15$ minutes. Weight and sex was recorded prior to liver excision. Liver tissue was collected from zebrafish by surgical manipulation under a stereoscopic microscope (Leica Microsystems) and stored at $-20^{\circ} \mathrm{C}$ for further use.

Exposure water samples $(100 \mathrm{~mL})$ were collected monthly throughout exposures. Control water samples $(100 \mathrm{~mL})$ were collected at the start, half-way and final month time points. Both exposure and control samples were immediately spiked with two internal standards (10ng/L). Samples were stored at $4{ }^{\circ} \mathrm{C}$ until further analysis.

The following compounds were used for LC-MS evaluation: 17 $\alpha$-ethynylestradiol (EE2) was obtained from Sigma-Aldrich. Formic acid, methanol (LC-MS grade) and water (LC-MS grade) were from Burdick and Jackson (Muskegon, MI). 17ß-Estradiol16, 16, 17-d3 (E2D3) was from Toronto Research Chemicals Inc. 17 $\alpha$-Ethynylestradiol2,4,16,16-d4 (EE2D4) was from CDN Isotopes.

Calibrators were generated to cover the concentration range of EE2 in mixing tank water from 0.1ng-100ng EE2/L using dilutions of authentic standard dried and

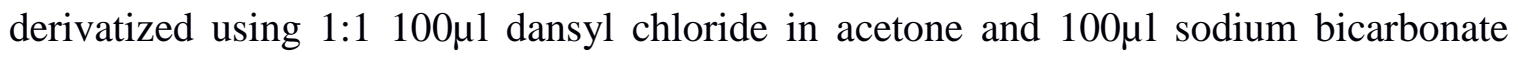
incubated at $60^{\circ} \mathrm{C}$ for 4 mins, cooled to room temperature as starting mobile phase.

LC-ESI-MS analyses were performed using a high-resolution $(30,000)$ Thermo LTQ-Orbitrap Discovery hybrid mass spectrometry instrument (San Jose, CA) equipped 
with an electrospray ionization source operating in the positive mode. The ESI interface using the following settings: sheath gas flow rate 40 (arbitrary units), aux gas flow rate 15 (arbitrary units), spray voltage $2 \mathrm{kV}$, capillary temperature $250 \mathrm{C}$, capillary voltage $-17 \mathrm{~V}$, and tube lens voltage $-85 \mathrm{~V}$. The Orbitrap was externally calibrated prior to data acquisition allowing accurate mass measurements for $[\mathrm{M}+\mathrm{H}]^{+}$or $[\mathrm{M}-\mathrm{H}]^{-}$ions to be obtained to within 4ppm.

The Orbitrap was coupled to a Thermo Accela HPLC system. EE2 was resolved using a Kinetex C18 (4.6x50mm, $2.1 \mathrm{~mm}$ i.d., 2.5uL particle size) HPLC column with guard cartridge (Phenomenex; Torrance, CA). Mobile phases consisted of $0.1 \%$ formic acid in HPLC grade water (A), $0.1 \%$ formic acid in acetonitrile (CAN) (B). The gradient mobile phase was delivered at a flow rate of $0.3 \mathrm{~mL} / \mathrm{min}$ and the formic:water mobile phase.

Start conditions were $30 \% \mathrm{~B}$, increased to $98 \%$ B over 7 minutes, decreased to $30 \% \mathrm{~B}$ over the next 2.5 minutes, $100 \% \mathrm{~B}$ over the next three minutes. Column was washed at $100 \% \mathrm{~B}$ for 1 minute and back to $30 \% \mathrm{~B}$ for 5 minutes. The column temperature was kept at $50^{\circ} \mathrm{C}$ using a thermostatic column oven. The sample injection volume was $20 \mu \mathrm{L}$.

\section{Results and Discussion}

We exposed 6 groups of full-sib offspring from 3 strains of zebrafish: $\mathrm{AB}, \mathrm{Tu}$, and WIK, across 5 treatment categories: 1ng, 10ng, 25ng, 50ng EE2/L and control (methanol $\leq 0.0005 \%$ ) to assess the effects throughout development and maturation, embryos to 6 months. Survival curves and sex ratios were examined after 6 month exposure. At 8 
weeks of age, a portion of juveniles were partitioned off to examine the gonadal tissue after exposure from fertilization through sexual differentiation.

We were interested in comparing the survivorship of each treatment type (1ng, 10ng, 25ng, and 50ng EE2/L) to control $(\leq 0.0005 \%$ methanol) using three zebrafish strains $(\mathrm{AB}, \mathrm{Tu}$ and $\mathrm{WIK})$ from fertilization through 6 week exposure then continued from 6 weeks to 6 month exposure.

Survivorship from fertilization through 6 weeks of exposure are shown for all treatment types: control, 1ng, 10ng, 25ng, and 50ng EE2/L (figure 27); however, the 50ng EE2/L treated fish for all strains and pairs had impaired growth with survival lower than $80 \%$ in 4 of 6 groups (pair/strain combo). Therefore the 50ng EE/L treatment was terminated at 8 weeks for all strain/pair combos as fish to prevent pain and suffering of surviving fish. In general the surviving fish did not appear healthy and exhibited (put abnormal traits observed here).

In all treatment groups, unaccounted loss (i.e., missed counts on tiny larvae and/or cannibalism) were shown on the last day of exposure. The greatest loss in all treatments occurred within the first 10 days post fertilization which is attributed to normal larval mortality. Control fish from 0-6 weeks had varied survival; however after 10 days post fertilization, the most fragile period of development, no strain/pair combo had greater than $10 \%$ loss over multiple day periods and loss beyond that was accounted for at the end of the 6 weeks. Similar patterns occurred from 0-6 weeks in 1ng, 10ng and 25ng EE2/L treatment groups; most loss occurring within the first 10 days and other major 
losses shown at the end of the 6 week survivorship attributed to miscounts and/or cannibalism.

For the 6 week- 6 month survival curves, 20 fish per tank were added and we therefore reset the percentage to $100 \%$. Survival during this period was consistent across all tanks and strains (Figures 27-31). As in the 0-6wks survival curves, any miscounts are demonstrated at the end of the 180 day exposure which was $<10 \%$ across all treatments. Among all strains and treatments a near $100 \%$ survival was observed. The exception to this was the 25ng EE2/L treated fish which exhibited between $70-90 \%$ survival in all strain/pair combos except 1, TuCH1. Phenotypically, 25ng EE2/L fish appeared to have similar appearance to other treatments despite survival patterns not demonstrating a similar pattern.

Among the $50 \mathrm{ng}$ EE2/L treatments, the greatest loss occurred weeks 6 and 7. No outstanding differences were observed between strains, but the $50 \mathrm{ng}$ EE2/L treated fish were not developing normally so treatments were terminated despite the absence of major losses to prevent undue pain and suffering. The greatest losses in the full exposure treatments from 6wks-6months occurred in the 25ng EE2/L despite no outwardly apparent developmental abnormalities and normal growth.

When evaluating the sex ratios among the surviving treatments, a trend was observed. The proportion of females increased along with treatment level (i.e., 1ng, 10ng, and 25ng EE2/L) compared to control (Figure 32). These differences resulted in a statistically significant difference in the proportion of females between control and 10ng EE2/L treated and control and 25ng EE2/L treated. 
Gross images were taken prior to fixation for histological processing to demonstrate morphological differences seen between control and treatment groups (Figures 33-36). Assessing three phenotypic categories, reduced body size, edema in body cavity and bulging eye (as seen in highest acute exposure treatment groups), we see that the 25ng and 50ng EE2/L treatment groups display abnormal growth patterns compared to control, 1ng and 10ng EE2/L treatments (Table 17) which is supported by our survival curves.

During processing, chemical resistant marker was used for labeling of cassettes; however, xylene used during processing resulted in loss of sample identification. No results can be reported on the whole fish mid-sagittal sections; however the slides were still assessed and general unique characteristics were seen. We observed distinct differences between slides including intersex gonadal tissue (Figure 38). In addition, we observed differential H\&E staining uptake demonstrated in male liver tissue between males exposed to estrogen vs unexposed (Ven et al., 2003). We observed similar proportions of male liver tissue with basophilia as EE2 treated proportions sent for histological processing.

Analysis of samples showed contamination which was sourced to be within the $100 \%$ methanol used for LC-MS specific solubilization. Samples could not be used for further analysis because this was the methanol stock used to prepare LC-MS EE2 and internal standard stocks; however, this was not the methanol used to prepare working EE2 stocks for spiking common mixing tanks. Despite contamination, we did find the 
dansyl chloride derivatization method to be much more effective in increasing sensitivity to EE2 quantification.

As a result of funding, we were unable to carry samples through library preparation and sequencing as described in chapter II; however, samples are being stored at $-20^{\circ} \mathrm{C}$ for future evaluation of the effects of chronic EE2 treatment from embryonic development through 6 months of age. We observed treatment specific effects despite not being able to analysis chronic treatment of EE2 on mitochondrial genome stability.

While we were not able to carry this study through its goal of examining the effects of chronic exposure of environmentally relevant concentrations of EE2 on three common strains of zebrafish from embryonic development to reproductive maturity, we were still able to gain valuable information from the exposure series of $1,10,25$, and 50ng EE2/L compared to our controls. Our results demonstrated normal development and growth at 1, 10, and 25ng EE2/L while toxicity at 50ng EE2/L in developing zebrafish was observed. We observed different female proportions between control versus our two highest concentrations of EE2 treatment, 10ng and 25ng EE2/L. When these results are taken into consideration with our acute exposure results of survivorship and female proportions, it supports the necessity behind further investigation of EE2 treatments at varied concentrations during different life stages of zebrafish development. 


\section{Figures and Tables}

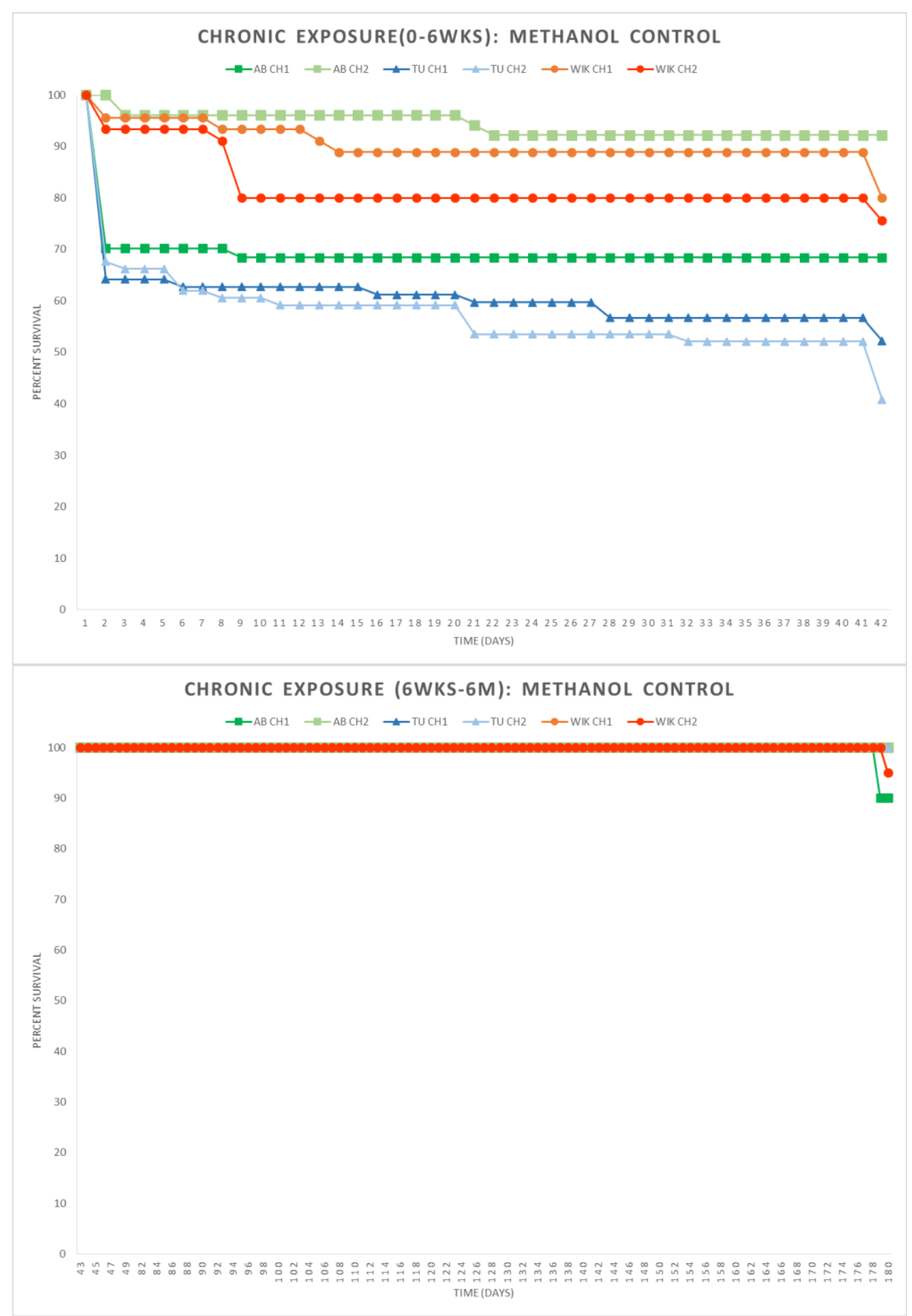

Figure 27: Percent survival against 6 month exposure in 0dpf-6weeks (top) and 6weeks-6months (bottom) for control groups of three zebrafish strains: AB (green), Tu (blue), and WIK (orange) from two pairs per strain. 


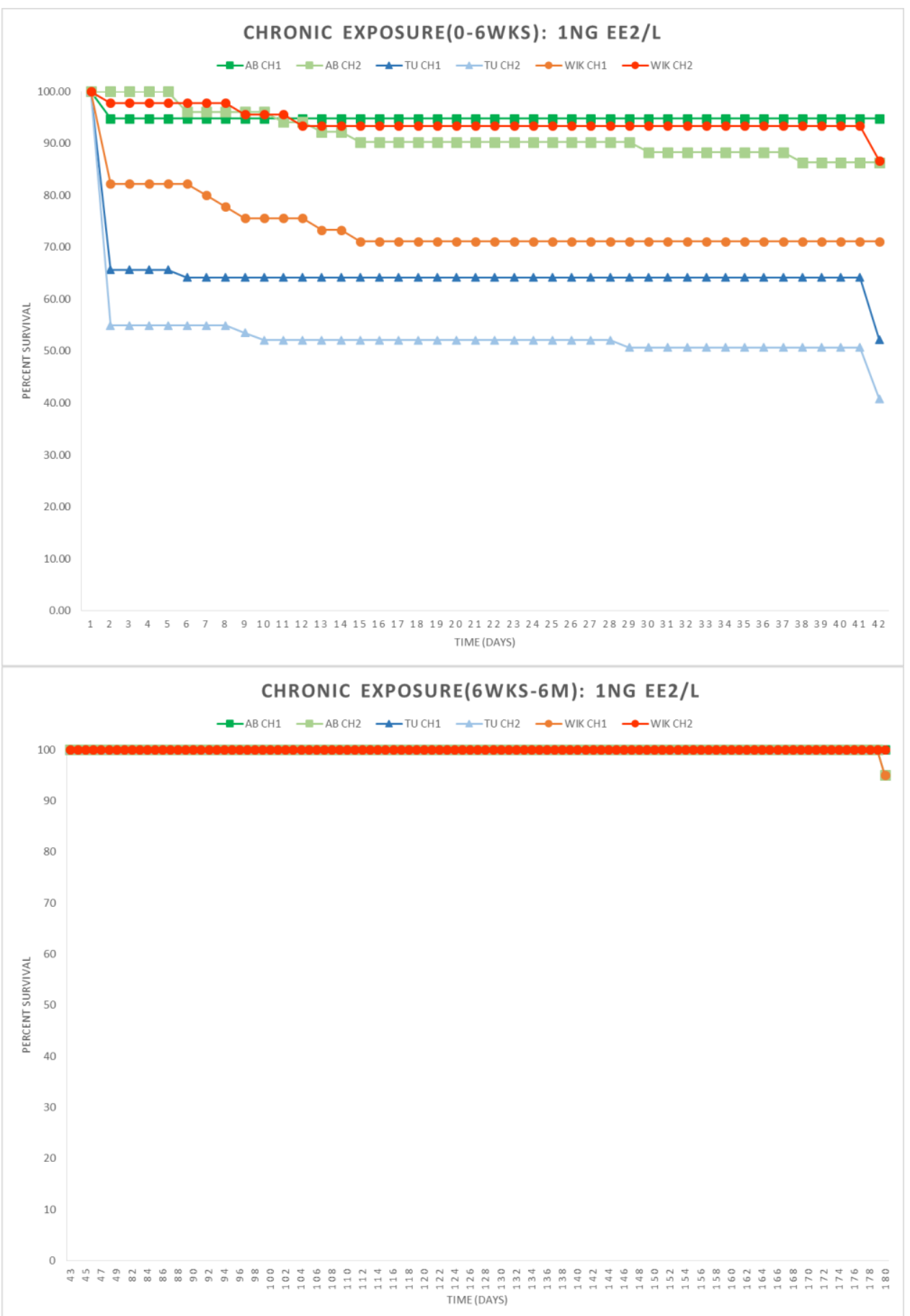

Figure 28: Percent survival against 6 month exposure in 0dpf-6weeks (top) and 6weeks-6months (bottom) for 1ng EE2/L treatment groups of three zebrafish strains: AB (green), Tu (blue), and WIK (orange) from two pairs per strain. 


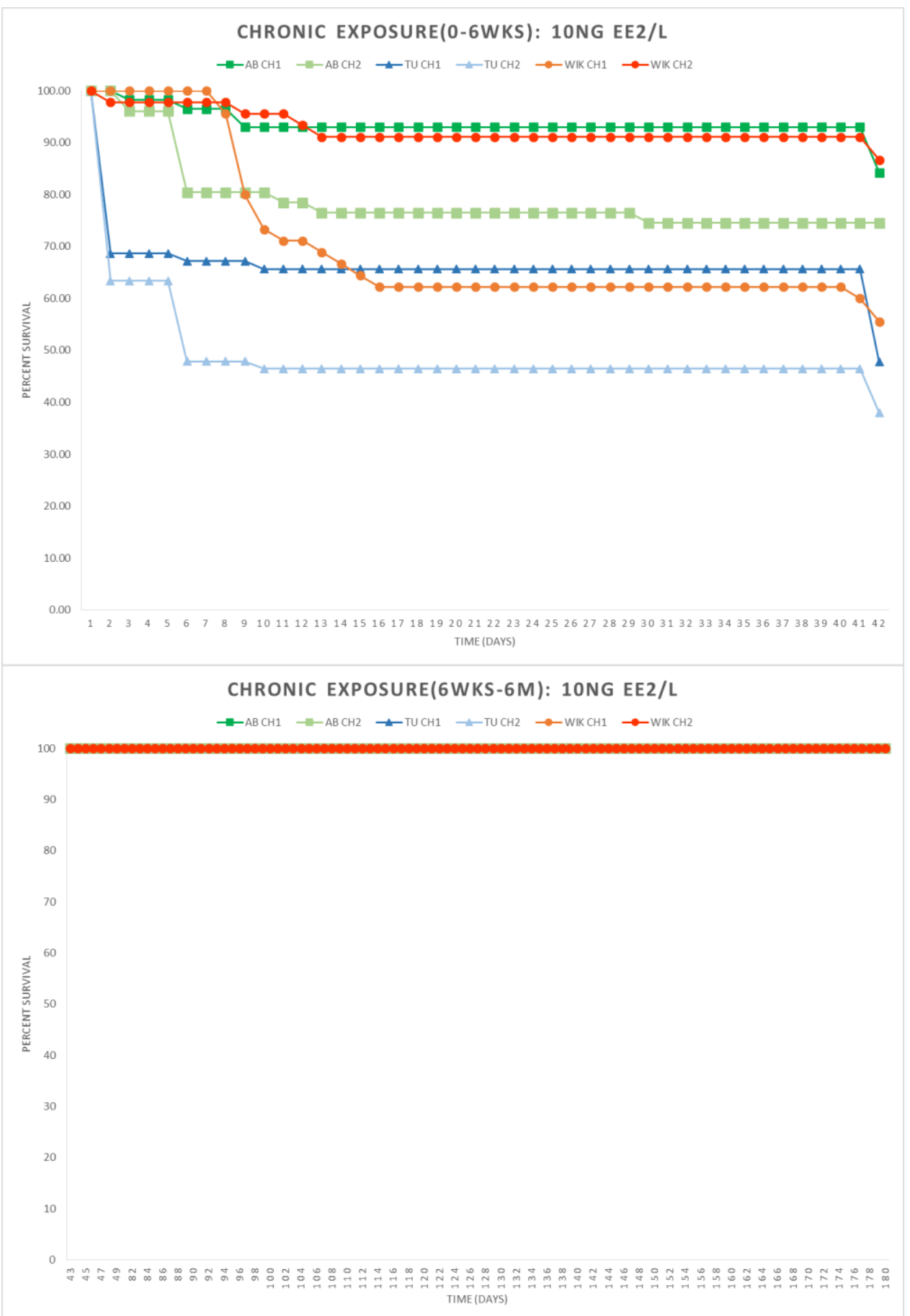

Figure 29: Percent survival against 6 month exposure in 0dpf-6weeks (top) and 6weeks-6months (bottom) for 10ng EE2/L treatment groups of three zebrafish strains: AB (green), Tu (blue), and WIK (orange) from two pairs per strain. 


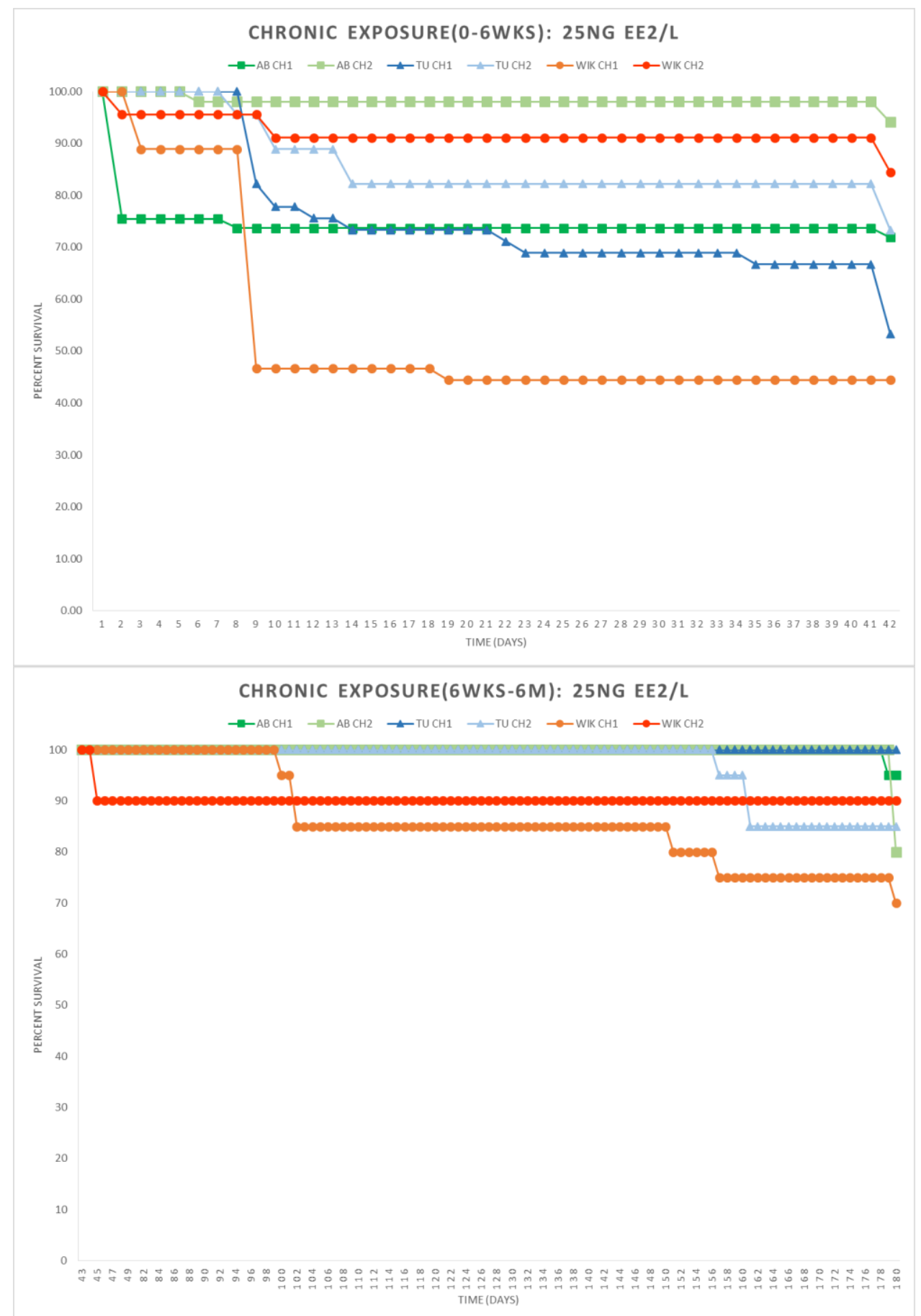

Figure 30: Percent survival against 6 month exposure in 0dpf-6weeks (top) and 6weeks-6months (bottom) for 25ng EE2/L treatment groups of three zebrafish strains: AB (green), Tu (blue), and WIK (orange) from two pairs per strain. 


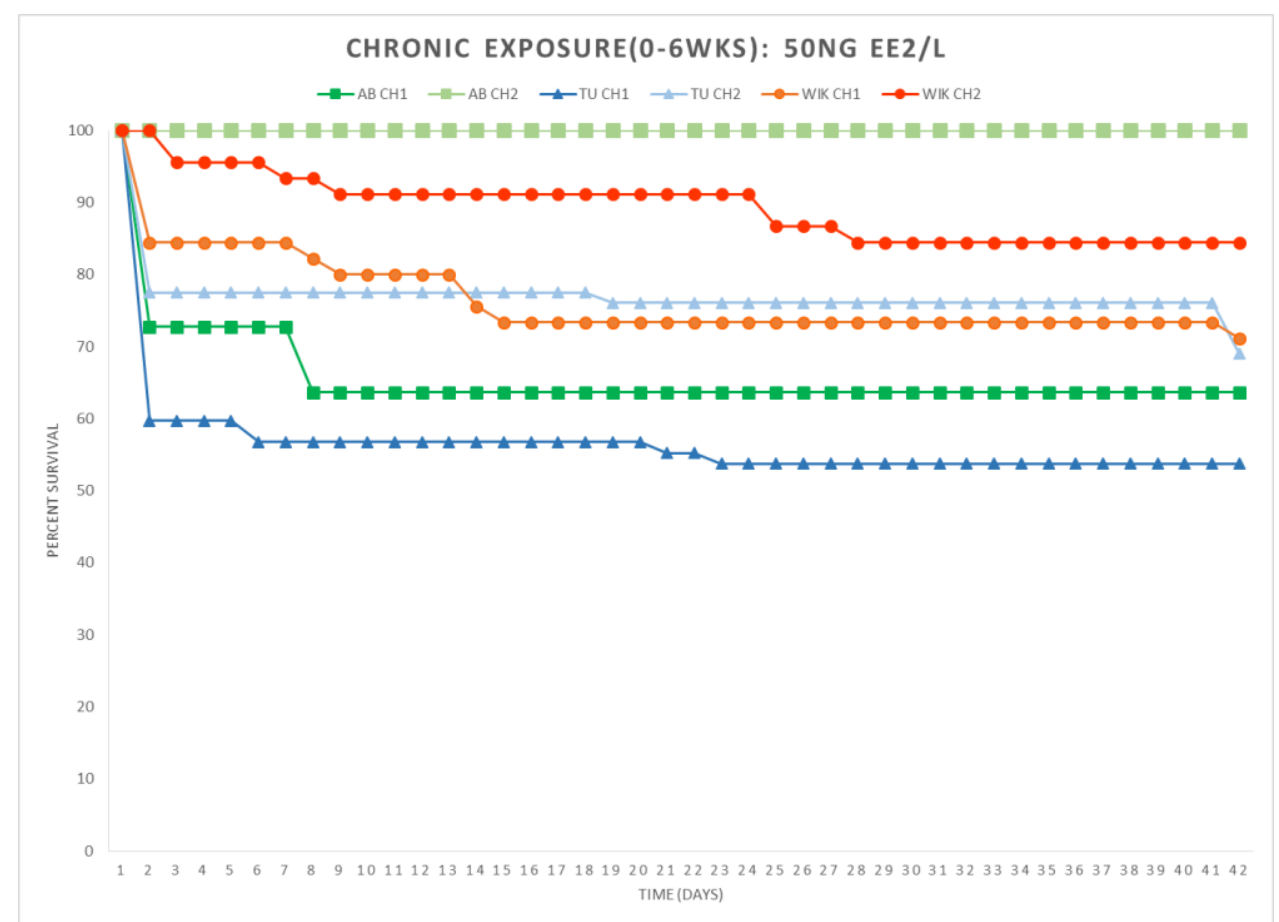

Figure 31: Percent survival against 6 month exposure in 0dpf-6weeks for 50ng EE2/L treatment groups of three zebrafish strains: AB (green), Tu (blue), and WIK (orange) from two pairs per strain.

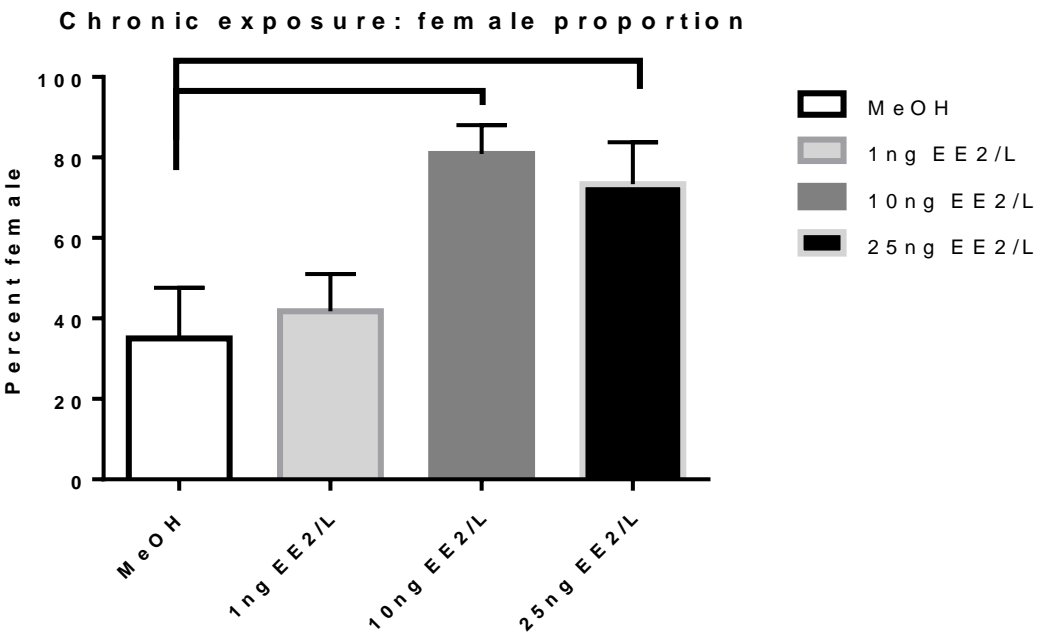

Controlvs treatment type

Figure 32: Proportion of females between control and 1ng, 10ng, and 25ng EE2/L treated fish after 6 month exposure starting from 5 hours post fertilization. 


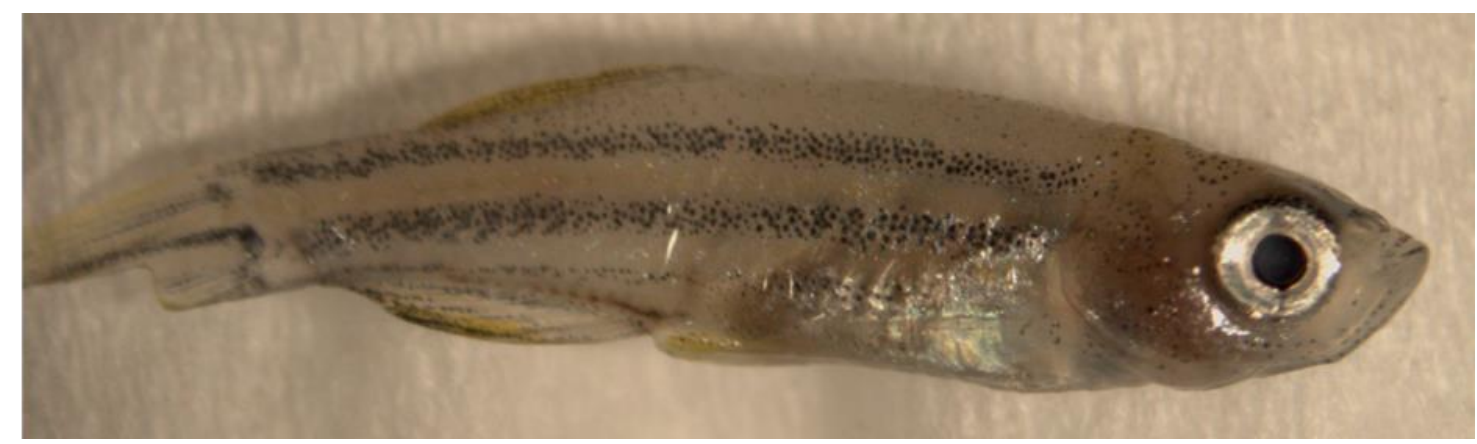

Figure 33: Gross image via stereoscopic microscope camera of control $(\leq 0.0005 \%$ methanol $)$ larva at 8 weeks

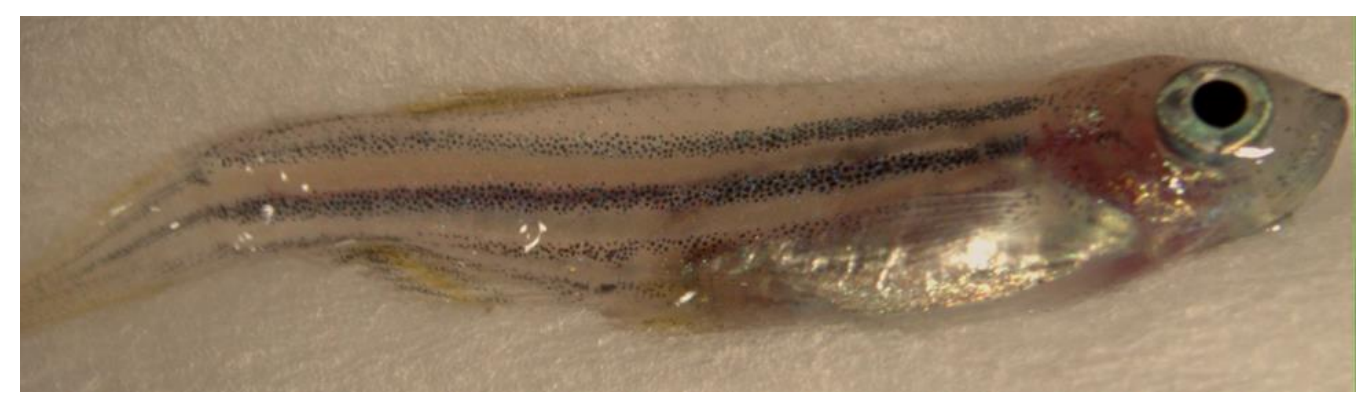

Figure 34: Gross images via stereoscopic microscope camera of 50ng EE2/L treated larva at 8 weeks demonstrating reduced body size and edema in body cavity.

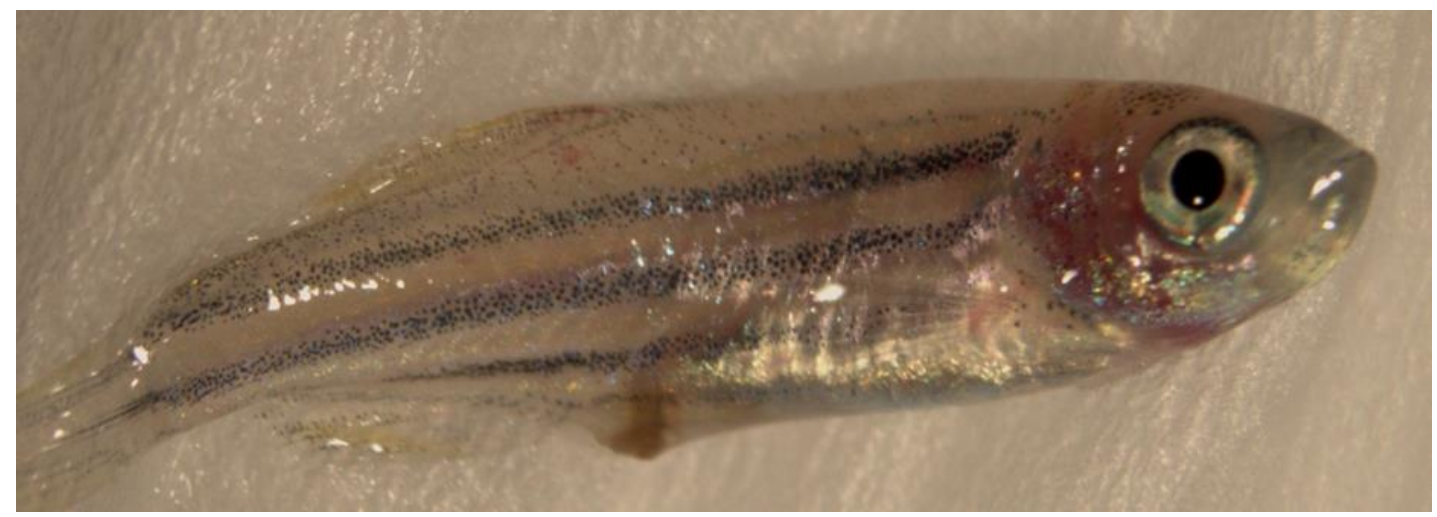

Figure 35: Gross image via stereoscopic microscope camera of 10ng EE2/L treated larva at 8 weeks 


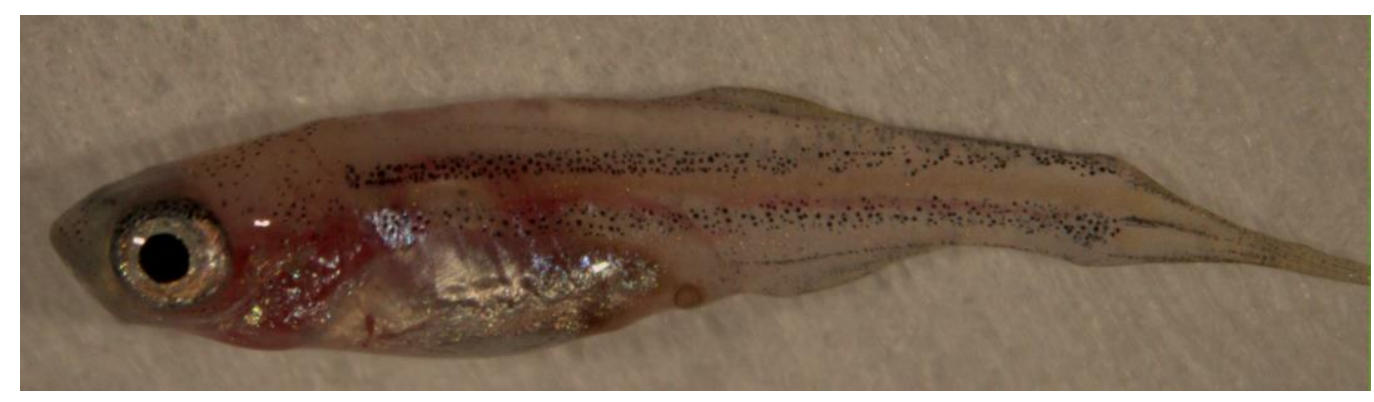

Figure 36: Gross image via stereoscopic microscope camera of 25ng EE2/L treated larva at 8 weeks

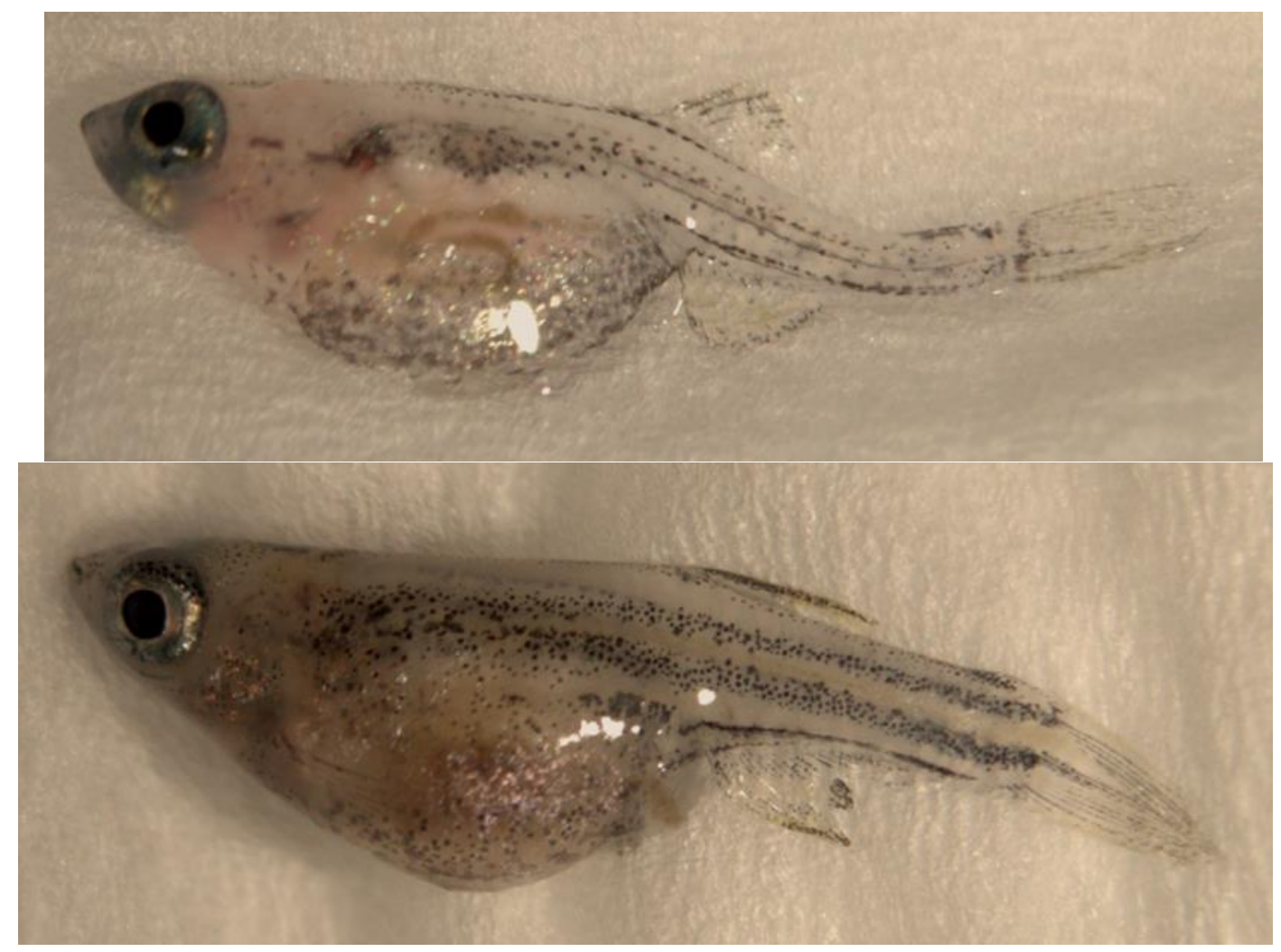

Figure 37: Gross images via stereoscopic microscope camera of 50ng EE2/L treated larva at 8 weeks demonstrating reduced body size and edema in body cavity. 
Table 17: Control vs treated at 8 weeks, phenotypic comparison of 3 developmental categories

\begin{tabular}{c|c|c|c|c|c}
\hline Phenotype & Control & 1ng EE2/L & 10ng EE2/L & 25ng EE2/L & 50ng EE2/L \\
\hline Reduced body size & No & No & No & Yes & Yes \\
\hline Edema in body cavity & No & No & No & No & Yes \\
\hline Bulging eye & No & No & No & No & Yes
\end{tabular}

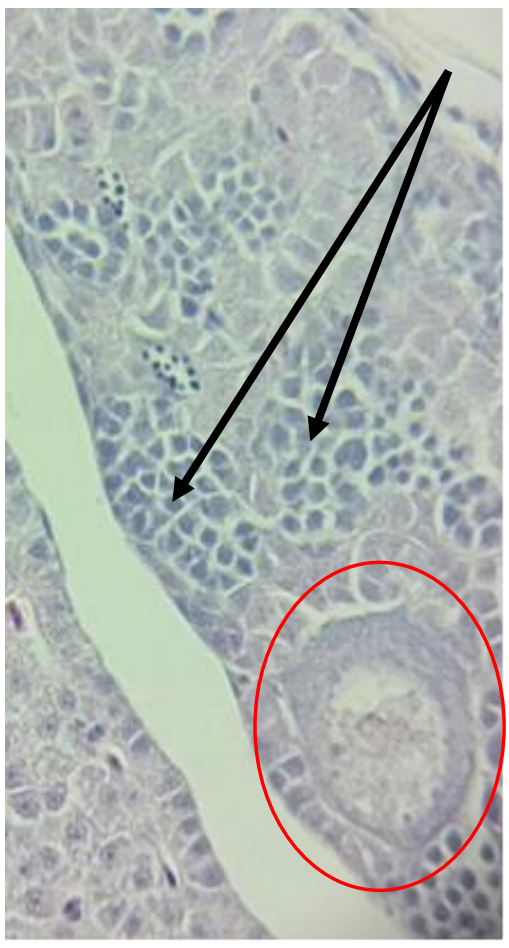

Figure 38: Histology image of intersex tissue from 8 week old (potentially EE2 exposed) fish larva (red circle indicating an oocyte and black arrows indicating testicular tissue). 


\section{Chapter V: Discussion}

This project aimed to assess the effects of environmentally relevant concentrations of $17 \alpha$-ethynylestradiol on mitochondrial genome stability in the model zebrafish. My central hypothesis was, "The environmental estrogen 17- $\alpha$ ethynylestradiol increases mitochondrial DNA heteroplasmy." Our investigation for testing this hypothesis revealed novel aspects of mutation accumulation within trout and among three strains of zebrafish evaluated. We demonstrated in two of our experimental investigations that environmentally relevant, low level exposure to EE2 increases mitochondrial heteroplasmy in two different species of fish. In addition to seeing effects on the mtgenome, we saw that both acute and chronic exposure to EE2 had severe effects of development and survival on zebrafish.

Further evaluation of this sequencing data is necessary to differentiate nonfunctional consequences from functional consequences. Of these increased heteroplasmic sites, which are associated directly to the exposure and of these which have the potential to, or currently decrease, mitochondrial function? In addition, continued investigation of environmental estrogen exposure on mtgenome stability is necessary for understanding the effects of exposure as well as the possible initiation of human disease state from exposure. We demonstrated that there is an overall increase in mitochondrial heteroplasmy under environmentally relevant exposure regimes, but what are the impactions for human health? Ultimately, can this eventually induce mitochondrial disease states? 
The results of this investigation provide the framework for studies involving toxicant exposure and mitochondrial genome stability. In this study we investigated a single toxicant; however, both aquatic and terrestrial vertebrates, including humans are exposed to a wealth of toxicants that have the potential to induce subtle and slow mutations within the mitochondrial genome. 


\section{References}

Ameur, A., Stewart, J. B., Freyer, C., Hagström, E., Ingman, M., Larsson, N.-G., \& Gyllensten, U. (2011). Ultra-deep sequencing of mouse mitochondrial DNA: mutational patterns and their origins. PLoS Genetics, 7(3), e1002028. http://doi.org/10.1371/journal.pgen.1002028

Broughton, R. E., Milam, J. E., \& Roe, B. a. (2001). The complete sequence of the zebrafish (Danio rerio) mitochondrial genome and evolutionary patterns in vertebrate mitochondrial DNA. Genome Research, 11(11), 1958-67. http://doi.org/10.1101/gr.156801

Broughton, R. E., Milam, J. E., \& Roe, B. A. (2001). The Complete Sequence of the Zebrafish ( Danio rerio ) Mitochondrial Genome and Evolutionary Patterns in Vertebrate Mitochondrial DNA, 1958-1967. http://doi.org/10.1101/gr.156801.1

Brown, K. (2008). Fish mitochondrial genomics: sequence, inheritance and functional variation. Journal of Fish Biology, 72(2), 355-374. http://doi.org/10.1111/j.10958649.2007.01690.x

Brown, K., Dobrinski, K., Lee, A., Gokcumen, O., Mills, R., Shi, X., ... Lee, C. (2012). Extensive genetic diversity and substructuring among zebrafish strains revealed through copy number variant analysis. PNAS, 109(2), 529-34. http://doi.org/10.1073/pnas.1112163109 
Brown, K., Schultz, I., Cloud, J., \& Nagler, J. (2008). Aneuploid sperm formation in rainbow trout exposed to the environmental estrogen 17 $\alpha$-ethynylestradiol. PNAS, 105(50), 19786-19791.

Brown, K., Schultz, I., \& Nagler, J. (2007). Reduced embryonic survival in rainbow trout resulting from paternal exposure to the environmental estrogen 17alphaethynylestradiol during late sexual maturation. Reproduction, 134(5), 659-66. http://doi.org/10.1530/REP-07-0169

Chan, D. C. (2006). Mitochondria: dynamic organelles in disease, aging, and development. Cell, 125(7), 1241-52. http://doi.org/10.1016/j.cell.2006.06.010

Chandra, D., \& Singh, K. K. (2011). Genetic insights into OXPHOS defect and its role in cancer. Biochimica et Biophysica Acta, 1807(6), 620-5. http://doi.org/10.1016/j.bbabio.2010.10.023

Cheng, Z., \& Ristow, M. (2013). Mitochondria and metabolic homeostasis. Antioxidants \& Redox Signaling, 19(3), 240-2. http://doi.org/10.1089/ars.2013.5255

Coe, T. S., Söffker, M. K., Filby, A. L., Hodgson, D., \& Tyler, C. R. (2010). Impacts of early life exposure to estrogen on subsequent breeding behavior and reproductive success in zebrafish. Environmental Science \& Technology, 44(16), 6481-7. http://doi.org/10.1021/es101185b 
Desbrow, C., \& Routledge, E. (1998). Identification of estrogenic chemicals in STW effluent. 1. Chemical fractionation and in vitro biological screening. Environmental ..., (97), 1549-1558. Retrieved from http://pubs.acs.org/doi/abs/10.1021/es9707973

Duchen, M. R. (2004). Roles of Mitochondria in Health and Disease, 53(February).

Falk, M. J., Pierce, E. A., Consugar, M., Xie, M. H., Guadalupe, M., Hardy, O., ... Gai, X. (2014). mitochondrial genome, 14(79), 389-399.

Fenske, M., Maack, G., Schäfers, C., \& Segner, H. (2005). An environmentally relevant concentration of estrogen induces arrest of male gonad development in zebrafish, Danio rerio. Environmental Toxicology and Chemistry / SETAC, 24(5), 1088-98. Retrieved from http://www.ncbi.nlm.nih.gov/pubmed/16110986

Flores, A., \& Hill, E. M. (2008). Formation of estrogenic brominated ethinylestradiol in drinking water: implications for aquatic toxicity testing. Chemosphere, 73(7), 111520. http://doi.org/10.1016/j.chemosphere.2008.07.022

Frezza, C., Cipolat, S., \& Scorrano, L. (2007). Organelle isolation : functional mitochondria from mouse liver, muscle and cultured filroblasts. Nature Protocols, 2(2), 287-295. http://doi.org/10.1038/nprot.2006.478

Gladyshev, V. N. (2013). The free radical theory of aging is dead. Long live the damage theory! Antioxidants \& Redox Signaling, 1-9. http://doi.org/10.1089/ars.2013.5228 
Goldstein, A. C., Bhatia, P., \& Vento, J. M. (2013). Mitochondrial Disease in Childhood : Nuclear Encoded, (March), 212-226. http://doi.org/10.1007/s13311-013-0185-6

Gray, M. W. (1999). Evolution of organellar genomes. Current Opinion in Genetics \& Development, 9(6), 678-87. Retrieved from http://www.ncbi.nlm.nih.gov/pubmed/10607615

Gray, M. W., Burger, G., \& Lang, B. F. (1999). Mitochondrial evolution. Science (New York, N.Y.), 283(5407), 1476-81.

Hamid, H., \& Eskicioglu, C. (2012). Fate of estrogenic hormones in wastewater and sludge treatment: A review of properties and analytical detection techniques in sludge matrix. Water Research, 46(18), 5813-33. http://doi.org/10.1016/j.watres.2012.08.002

He, Y., Wu, J., Dressman, D. C., Iacobuzio-donahue, C., Markowitz, S. D., Velculescu, V. E., ... Papadopoulos, N. (2010). Heteroplasmic mitochondrial DNA mutations in normal and tumour cells. Nature, 464(7288), 610-614. http://doi.org/10.1038/nature08802

Hecker, M., \& Hollert, H. (2011). Endocrine disruptor screening: regulatory perspectives and needs. Environmental Sciences Europe, 23(1), 15. http://doi.org/10.1186/2190$4715-23-15$ 
Howe, K., Clark, M. D., Torroja, C. F., Torrance, J., Berthelot, C., Muffato, M., ... Stemple, D. L. (2013). The zebrafish reference genome sequence and its relationship to the human genome. Nature, 496(7446), 498-503. http://doi.org/10.1038/nature12111

Hunt, P., \& Hassold, T. (2008). Human female meiosis: what makes a good egg go bad? Trends in Genetics, 24(2), 86-93. http://doi.org/10.1016/j.tig.2007.11.010

Hunt, P., Koehler, K., Susiarjo, M., Hodges, C., Ilagan, A., Voigt, R., ... Carolina, N. (2003). Bisphenol A Exposure Causes Meiotic Aneuploidy in the Female Mouse. Current Biology, 13(August 1998), 546-553. http://doi.org/10.1016/S

Ingerslev, F., Vaclavik, E., \& Halling-Sørensen, B. (2003). Pharmaceuticals and personal care products-A source of endocrine disruption in the environment? Pure and Applied Chemistry. Retrieved from http://www.iupac.org/publications/pac/75/11/1881/

Jayaprakash, A. D., Benson, E., Liang, R., Shim, J., Lambertini, L., Aaronson, S. A., \& Sachidanandam, R. (2014). Heteroplasmy is ubiquitous and stable at the single cell level, 1-15.

Kaipparettu, B. A., Ma, Y., Park, J. H., Lee, T.-L., Zhang, Y., Yotnda, P., .. Wong, L.-J. C. (2013). Crosstalk from non-cancerous mitochondria can inhibit tumor properties of metastatic cells by suppressing oncogenic pathways. PloS One, 8(5), e61747. http://doi.org/10.1371/journal.pone.0061747 
Kelly, D. P., \& Scarpulla, R. C. (2004). Transcriptional regulatory circuits controlling mitochondrial biogenesis and function. Genes \& Development, 18(4), 357-68. http://doi.org/10.1101/gad.1177604

Kidd, K. a, Blanchfield, P. J., Mills, K. H., Palace, V. P., Evans, R. E., Lazorchak, J. M., \& Flick, R. W. (2007). Collapse of a fish population after exposure to a synthetic estrogen. PNAS, 104(21), 8897-901. http://doi.org/10.1073/pnas.0609568104

Kolpin, D. W., Furlong, E. T., Meyer, M. T., Thurman, E. M., Zaugg, S. D., Barber, L. B., \& Buxton, H. T. (2002). Pharmaceuticals, hormones, and other organic wastewater contaminants in U.S. streams, 1999-2000: a national reconnaissance. Environmental Science \& Technology, 36(6), 1202-11. Retrieved from http://www.ncbi.nlm.nih.gov/pubmed/11944670

Kroemer, G., Galluzzi, L., Editors, G., Galluzzi, L., Kepp, O., Trojel-hansen, C., \& Kroemer, G. (2012). Mitochondrial Control of Cellular Life, Stress, and Death, 1198-1208. http://doi.org/10.1161/CIRCRESAHA.112.268946

Liew, W. C. (2013). Zebrafish sex : a complicated affair, 13(2), 172-187. http://doi.org/10.1093/bfgp/elt041

Liew, W. C., Bartfai, R., Lim, Z., Sreenivasan, R., \& Siegfried, K. R. (2012). Polygenic Sex Determination System in Zebrafish, 7(4). http://doi.org/10.1371/journal.pone.0034397 
Menuet, a, Le Page, Y., Torres, O., Kern, L., Kah, O., \& Pakdel, F. (2004). Analysis of the estrogen regulation of the zebrafish estrogen receptor (ER) reveals distinct effects of ERalpha, ERbeta1 and ERbeta2. Journal of Molecular Endocrinology, 32(3), 975-86. Retrieved from http://www.ncbi.nlm.nih.gov/pubmed/15171726

Meyer, J. N., Leung, M. C. K., Rooney, J. P., Sendoel, A., Hengartner, M. O., Kisby, G. E., \& Bess, A. S. (2013). Mitochondria as a target of environmental toxicants. Toxicological Sciences : An Official Journal of the Society of Toxicology, 134(1), 117. http://doi.org/10.1093/toxsci/kft102

Nunnari, J., \& Suomalainen, A. (2012). Mitochondria: in sickness and in health. Cell, 148(6), 1145-59. http://doi.org/10.1016/j.cell.2012.02.035

Payne, B. a I., Wilson, I. J., Yu-Wai-Man, P., Coxhead, J., Deehan, D., Horvath, R., ... Chinnery, P. F. (2013). Universal heteroplasmy of human mitochondrial DNA. Human Molecular Genetics, 22(2), 384-90. http://doi.org/10.1093/hmg/dds435

Pollock, M. S., Dubé, M. G., \& Schryer, R. (2010). Investigating the link between pulp mill effluent and endocrine disruption: attempts to explain the presence of intersex fish in the Wabigoon River, Ontario, Canada. Environmental Toxicology and Chemistry, 29(4), 952-65. http://doi.org/10.1002/etc.118 
Rogan, W. J., \& Gladen, B. C. (1985). Study of human lactation for effects of environmental contaminants: the North Carolina Breast Milk and Formula Project and some other ideas. Environmental Health Perspectives, 60(6), 215-21. Retrieved from

http://www.pubmedcentral.nih.gov/articlerender.fcgi?artid=1568567\&tool=pmcentre z\&rendertype $=a b s t r a c t$

Ropero, A. B., Alonso-Magdalena, P., Ripoll, C., Fuentes, E., \& Nadal, A. (2006). Rapid endocrine disruption: environmental estrogen actions triggered outside the nucleus. The Journal of Steroid Biochemistry and Molecular Biology, 102(1-5), 163-9. http://doi.org/10.1016/j.jsbmb.2006.09.019

Schapira, A. H. V. (2006). Mitochondrial disease. Lancet, 368(9529), 70-82. http://doi.org/10.1016/S0140-6736(06)68970-8

Schmitt, M. W., Kennedy, S. R., Salk, J. J., Fox, E. J., Hiatt, J. B., \& Loeb, L. A. (2012). Detection of ultra-rare mutations by next-generation sequencing, 2012, 1-6. http://doi.org/10.1073/pnas.1208715109//DCSupplemental.www.pnas.org/cgi/doi/10.1073/pnas.1208715109

Schultz, I. R., Orner, G., Merdink, J. L., \& Skillman, A. (2001). Dose - response relationships and pharmacokinetics of vitellogenin in rainbow trout after intravascular administration of 17 a -ethynylestradiol, 51, 305-318. 
Schultz, I. R., Skillman, A., Nicolas, J.-M., Cyr, D. G., \& Nagler, J. J. (2003). Short-term exposure to 17 alpha-ethynylestradiol decreases the fertility of sexually maturing male rainbow trout (Oncorhynchus mykiss). Environmental Toxicology and Chemistry/SETAC, 22(6), 1272-80. Retrieved from http://www.ncbi.nlm.nih.gov/pubmed/12785584

Shyu, C., Cavileer, T. D., Nagler, J. J., \& Ytreberg, F. M. (2011). Computational estimation of rainbow trout estrogen receptor binding affinities for environmental estrogens. Toxicology and Applied Pharmacology, 250(3), 322-6. http://doi.org/10.1016/j.taap.2010.11.005

Soares, J., Coimbra, a M., Reis-Henriques, M. a, Monteiro, N. M., Vieira, M. N., Oliveira, J. M. a, ... Santos, M. M. (2009). Disruption of zebrafish (Danio rerio) embryonic development after full life-cycle parental exposure to low levels of ethinylestradiol. Aquatic Toxicology (Amsterdam, Netherlands), 95(4), 330-8. http://doi.org/10.1016/j.aquatox.2009.07.021

Spinazzola, A., \& Zeviani, Æ. M. (2007). Disorders of Nuclear-Mitochondrial Intergenomic Communication, 39-51. http://doi.org/10.1007/s10540-007-9036-1

Susiarjo, M., Hassold, T. J., Freeman, E., \& Hunt, P. a. (2007). Bisphenol A exposure in utero disrupts early oogenesis in the mouse. PLoS Genetics, 3(1), e5. http://doi.org/10.1371/journal.pgen.0030005 
Takahashi, O., \& Oishi, S. (2006). Male reproductive toxicity of four bisphenol antioxidants in mice and rats and their estrogenic effect. Archives of Toxicology, 80(4), 225-41. http://doi.org/10.1007/s00204-005-0033-5

Ternes, T. a, Stumpf, M., Mueller, J., Haberer, K., Wilken, R. D., \& Servos, M. (1999). Behavior and occurrence of estrogens in municipal sewage treatment plants--I. Investigations in Germany, Canada and Brazil. The Science of the Total Environment, 225(1-2), 81-90.

Timmis, J. N., Ayliffe, M. a, Huang, C. Y., \& Martin, W. (2004). Endosymbiotic gene transfer: organelle genomes forge eukaryotic chromosomes. Nature Reviews. Genetics, 5(2), 123-35. http://doi.org/10.1038/nrg1271

Tyler, C., \& Jobling, S. (2008). Roach, Sex, and Gender-Bending Chemicals: The Feminization of Wild Fish in English Rivers. BioScience, 58(11), 1051-1059.

Tyler, C., Jobling, S., \& Sumpter, J. (1998). Endocrine disruption in wildlife: a critical review of the evidence. Critical Reviews in Toxicology, 28(4), 319-61. http://doi.org/10.1080/10408449891344236

Ven, L. T. M. Van Der, Holbech, H., Fenske, M., Van, E., Brandhof, D., Gielis-proper, F. K., \& Wester, P. W. (2003). Vitellogenin expression in zebrafish Danio rerio : e v aluation by histochemistry, immunohistochemistry, and in situ mRNA hybridisation, 65, 1-11. http://doi.org/10.1016/S0166-445X(03)00103-6 
Wallace, D. C., \& Chalkia, D. (2016). Mitochondrial DNA Genetics and the Heteroplasmy Conundrum in Evolution and Disease.

Wallace, D. C., \& Fan, W. (2011). Energetics, Epigenetics, Mitochondrial Genetics, 10(1), 12-31. http://doi.org/10.1016/j.mito.2009.09.006.ENERGETICS

Wallace, D. C., Fan, W., \& Procaccio, V. (2011). Mitochondria Energetics and Therapeutics, 297-348. http://doi.org/10.1146/annurev.pathol.4.110807.092314.Mitochondrial

Youle, R. J., Bliek, A. M. Van Der, Complementation, F. P., Mitochondria, B. D., Fusion, M., \& Proteins, F. (2012). REVIEW Mitochondrial Fission, Fusion, and Stress. Science, 337.

Young, W. P., \& Wheeler, P. A. (1992). DNA Fingerprinting Confirms Isogenicity of Androgenetically Derived Rainbow Trout Lines. 